\title{
TRIPLY COUPLED BENDING-BENDING-TORSION VIBRATIONAL ANALYSIS OF ROTATING BEAMS USING FEM AND DYNAMIC FINITE ELEMENT FORMULATION
}

\author{
By \\ Mohammad Shavezipur \\ B.Sc. in Mechanical Engineering, \\ Sharif University of Technology, Iran, 1993 \\ M.Sc. in Mechanical Engineering, \\ Amir Kabir University of Technology, Iran, 1996
}

\author{
A thesis \\ presented to Ryerson University \\ in partial fulfillment of the requirements for the degree of \\ Master of Applied Science \\ in the Program of \\ Mechanical Engineering
}

Toronto, Ontario, Canada, 2004

Mohammad Shavezipur $2004^{\circledR}$ 
UMI Number: EC53468

\section{INFORMATION TO USERS}

The quality of this reproduction is dependent upon the quality of the copy submitted. Broken or indistinct print, colored or poor quality illustrations and photographs, print bleed-through, substandard margins, and improper alignment can adversely affect reproduction.

In the unlikely event that the author did not send a complete manuscript and there are missing pages, these will be noted. Also, if unauthorized copyright material had to be removed, a note will indicate the deletion.

\section{UMI}

UMI Microform EC53468

Copyright 2009 by ProQuest LLC

All rights reserved. This microform edition is protected against unauthorized copying under Title 17, United States Code.

ProQuest LLC

789 East Eisenhower Parkway

P.O. Box 1346

Ann Arbor, MI 48106-1346 
Author's Declaration:

I hereby declare that I am the sole author of this thesis.

I authorize Ryerson University to lend this thesis to other institutions or individuals for the purpose of scholarly research.

I further authorize Ryerson University to reproduce this thesis by photocopying or by other means, in total or in part, at the request of other institutions or individuals for the purpose of scholarly research. 


\title{
TRIPLY COUPLED BENDING-BENDING-TORSION VIBRATIONAL ANALYSIS \\ OF ROTATING BEAMS USING FEM AND DYNAMIC FINITE ELEMENT FORMULATION
}

\author{
Master of Applied Science in the Program of \\ Mechanical Engineering \\ 2004 \\ MOHAMMAD SHAVEZIPUR \\ School of Graduate Studies \\ Department of Mechanical Engineering \\ Ryerson University
}

\begin{abstract}
This research presents the numerical analysis of the triply coupled flap-wise, cord-wise and torsional vibrations of flexible rotating blades. Euler-Bernoulli bending and St. Venant torsion beam theories are considered to derive the governing differential equations of motion. Based on Finite Element Methodology (FEM), the cubic "Hermite" shape functions are implemented where the solution of the equations results in a linear eigenproblem. Then, the Dynamic (frequency dependent) Trigonometric Shape Functions (DTSF's) for beam's uncoupled displacements are derived. The application of the Dynamic Finite Element (DFE) approach to the solution of the governing equations is then presented. The DFE formulation, based on the weighted residual method and the DTSF's, results in a nonlinear eigenproblem representing eigenvalues and eigenmodes of the system. The applicability of the DFE method is then demonstrated by illustrative examples, where a Wittrick-Williams root counting technique is used to find the system's natural frequencies. The DFE approach, an intermediate method between FEM and "Exact" formulation, is characterized by higher convergence rates, and can be advantageously used when multiple natural frequencies and/or higher modes of beam-like structures are to be evaluated.
\end{abstract}




\section{Borrower's page}

Ryerson University requires the signatures of all persons using or photocopying this thesis. Please sign below, and give address and date. 
Dedicated to my life....

My wife, Farnaz 


\section{Acknowledgements}

I wish to express my gratitude to my supervisor Dr. Seyed M. Hashemi for his significant guidance and valuable advice during my graduate studies at Ryerson University. Dr. Hashemi has helped me to have the right direction in my research and to get a better understanding about numerical analysis of mechanical systems in general and vibration analysis in particular.

I also would like to acknowledge the scholarship awarded by Ryerson University and the support provided by Natural Science and Engineering Research Council of Canada (NSERC). 


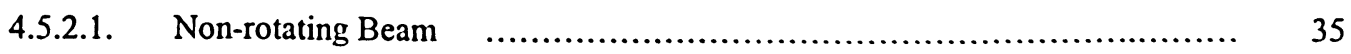

4.5.2.2. Axially Loaded Beam $\quad$..................................................... $\quad 38$

4.5.2.3. Non-rotating Pre-Twisted Beam $\quad$......................................... 41

4.5.3. Coupled Bending-Bending Vibrations $\quad$.......................................... 42

4.5.4. Coupled Bending-Bending-Torsion Vibrations $\quad$................................. 49

4.5.4.1. Vibrations of Non-rotating Uncoupled Beam $\quad$............................... 49

4.5.4.2. Vibrations of Non-rotating Coupled Beam $\quad$................................ 50

4.5.4.3. Vibrations of Rotating Triply Coupled Beam $\quad$............................. 52

CHAPTER 5: DYNAMIC FINITE ELEMENT METHOD $\quad$................................... 56

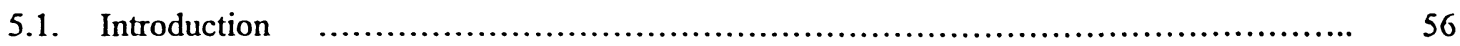

5.2. Frequency Dependent Approximation Functions $\quad$.................................... 56

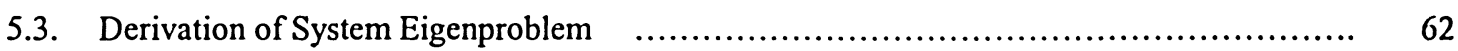

5.4. Refined Dynamic Finite Element (RDFE) ....................................... 65

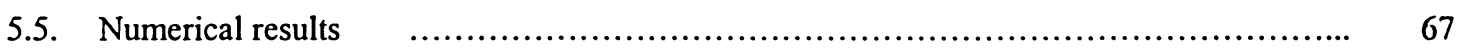

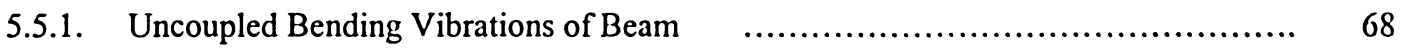

5.5.2. Coupled Bending-Torsion Vibrations $\quad$.......................................... 70

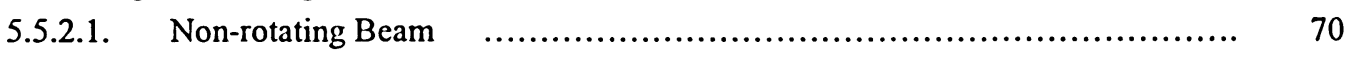

5.5.2.2. Axially Loaded Coupled Beam $\quad$......................................... $\quad 75$

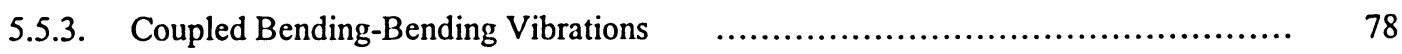

5.5.3.1. Axially Loaded Beam $\quad$................................................. 79

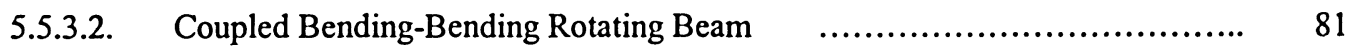

5.5.3.3. Rotating Beam with Variable Cross Section $\quad$................................. 86

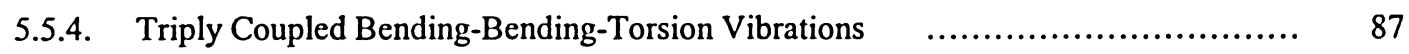

5.5.4.1. Uncoupled Beam Vibrations $\quad$............................................. 87

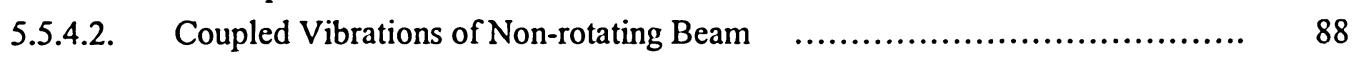

5.5.4.3. Vibrations of Rotating Triply Coupled Beam $\quad$............................ 93

CHAPTER 6: CONCLUSION _........................................................................ 99

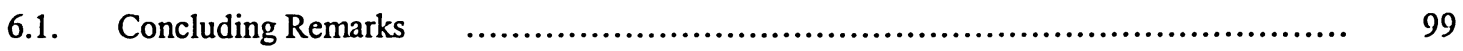

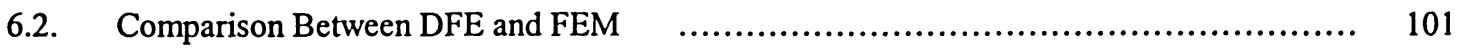

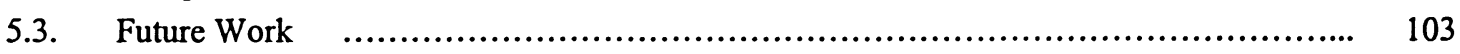

APPENDIX A: ANALYTICAL SOLUTION FOR BEAMS UNCOUPLED VIBRATIONS $\quad \ldots \quad 104$

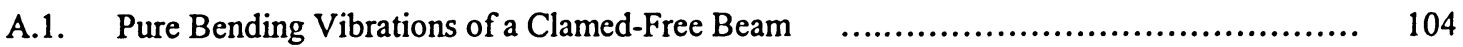

A.2. Torsional Vibrations of a Cantilever Beam $\quad$......................................... 107

APPENDIX B: COMPUTER PROGRAM, ALGORITHMS AND FUNCTIONS $\quad$............. 110

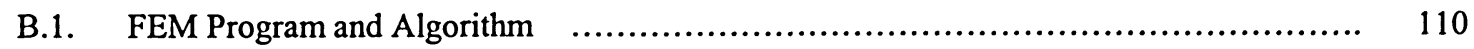

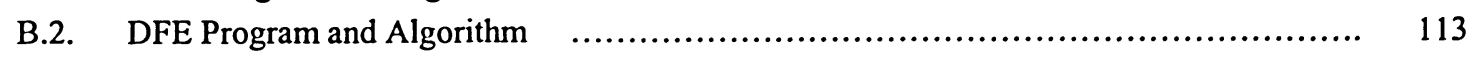

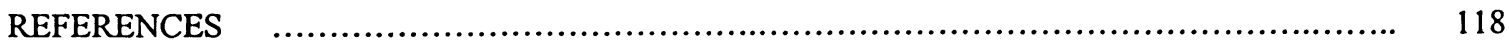


Author's Declaration

Borrower's Page

Abstract

Acknowledgements

Dedication

Tables of Contents vii

List of Tables ix

List of Illustrations

Nomenclature xiii

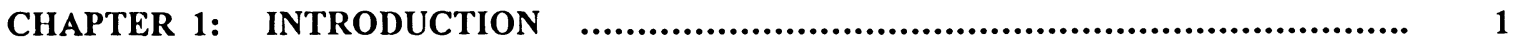

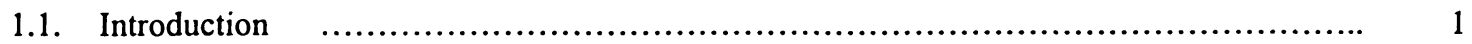

1.2. Objective

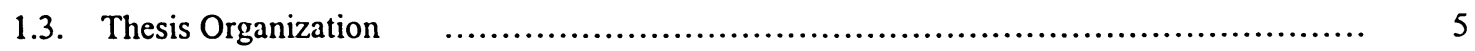

CHAPTER 2: $\quad$ EQUATIONS OF MOTION GOVERNING COUPLED VIBRATIONS OF $\quad . . \quad 7$

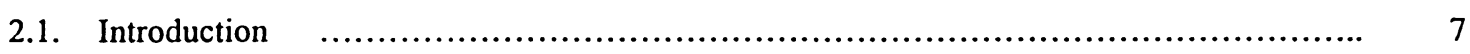

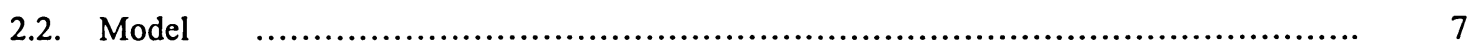

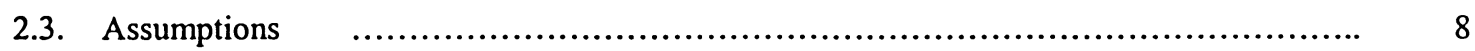

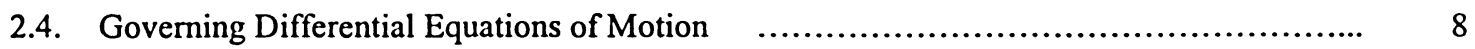

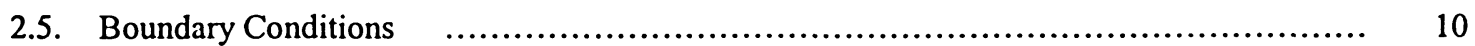

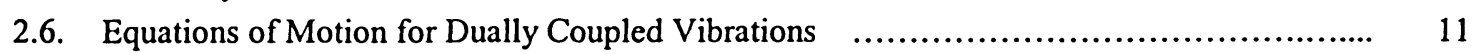

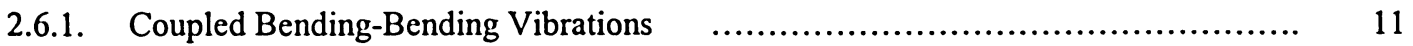

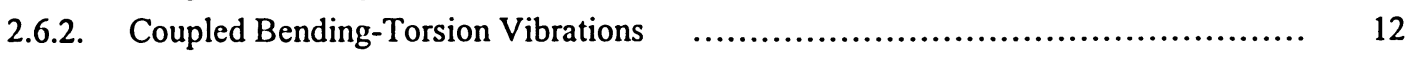

CHAPTER 3: SOLUTION OF EIGENPROBLEMS $\quad$......................................... 14

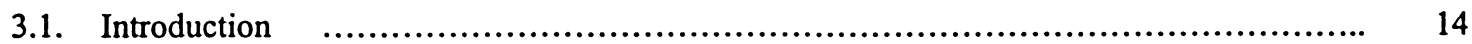

3.2. Wittrick-Williams Method $\quad$.......................................................... 15

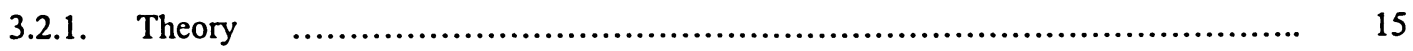

3.2.2. Application to the Beam Vibrations $\quad$......................................... 17

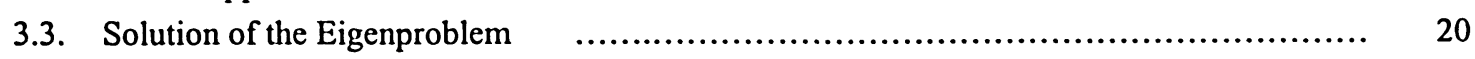

CHAPTER 4: FINITE ELEMENT METHOD $\quad$............................................ 22

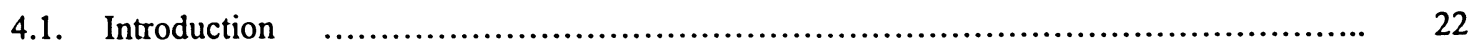

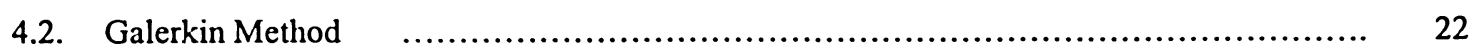

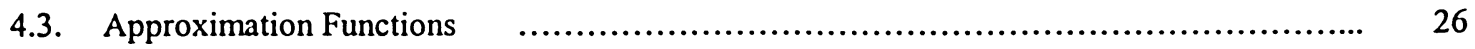

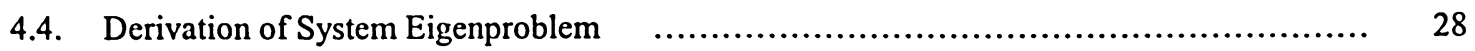

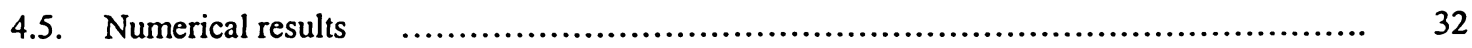

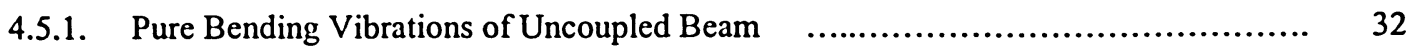

4.5.2. Coupled Bending-Torsion Vibrations $\quad$....................................... 34 


\section{List of Tables:}

Table 4.1: The first five natural frequencies of out-of-plane vibration of a cantilever beam

Table 4.2: The first six natural frequencies of coupled bending-torsion vibrations of a cantilever beam

Table 4.3: The mechanical and geometrical parameters of the three-beam wing

Table 4.4: The first five natural frequencies of the three-beam aircraft wing

Table 4.5: The bending-torsion natural frequencies of the cantilever beam (no axial load $(T=0)$ )

Table 4.6: The bending-torsion natural frequencies of the axially loaded cantilever beam $(T=-1790)$

Table 4.7: The bending-torsion natural frequencies of the pre-twisted clamped-free beam

Table 4.8: The properties of the scaled propeller blade model WADC S-5, with hub radius $e_{l}=6$

(in) and blade length $L=18$ (in)

Table 4.9: The natural frequencies $(\mathrm{Hz})$ of the first three modes of vibrations of propeller blade WADC S-5

Table 4.10: The uncoupled bending, bending and torsion natural frequencies of the clamped-free beam

Table 4.11: The coupled bending-bending-torsion natural frequencies of the pre-twisted cantilever beam

Table 4.12: The bending-bending-torsion natural frequencies of the pre-twisted cantilever rotating blade, $\Omega=0$ (rpm)

Table 4.13: The bending-bending-torsion natural frequencies of the pre-twisted cantilever rotating blade, $\Omega=360$ (rpm)

Table 5.1: The first five natural frequencies of out-of-plane vibration of a cantilever beam calculated by DFE

Table 5.2: The first six natural frequencies of coupled bending-torsion vibrations of a cantilever beam

Table 5.3: The first five natural frequencies of the three-beam aircraft wing obtained by DFE

Table 5.4: The bending-torsion coupled natural frequencies of the clamped-free beam

Table 5.5: The bending-torsion natural frequencies of the cantilever beam (no axial load $(T=0)$ )

Table 5.6: The bending-torsion natural frequencies of the axially loaded cantilever beam $(T=-1790 \mathrm{~N})$

Table 5.7: The uncoupled in- and out-of-plane bending natural frequencies of the cantilever beam 
Table 5.8: The coupled bending-bending natural frequencies of a rotating cantilever beam, $\mathbf{\Omega}=5(\mathrm{rad} / \mathrm{s})$

Table 5.9: The coupled bending-bending natural frequencies of the rotating beam

Table 5.10: The first three natural frequencies $(\mathrm{Hz})$ of vibrations of propeller blade $W A D C S-5$ (DFE method)

Table 5.11: The triply coupled bending-bending-torsion natural frequencies of the pre-twisted clamped-free beam

Table 5.12: The triply coupled bending-bending-torsion natural frequencies of the pre-twisted rotating beam, $\Omega=360$ (rpm)

Table 5.13: The triply coupled bending-bending-torsion natural frequencies of the twisted rotating blade, $\Omega=360$ ( $\mathrm{ppm}$ )

Table 5.14: The triply coupled bending-bending-torsion natural frequencies of the piecewise uniform twisted rotating blade, $\Omega=360(\mathrm{rpm})$

Table A.1: The weighted and normalized natural frequencies, $\alpha_{n} L$ and $\omega_{n}$, for a cantilever beam obtained by analytical solution 


\section{List of Figures:}

Figure 2.1: The beam configuration and geometrical parameters

Figure 3.1: The determinant of stiffness matrix versus frequency

Figure 3.2: The sign count of stiffness matrix versus frequency

Figure 3.3: The variation of $j_{f, w}$ versus frequency

Figure 4.1: The element's nodal variables

Figure 4.2: The transformation from physical coordinate to reference element coordinate

Figure 4.3: The first three modes of out-of-plane vibrations of a cantilever beam based on FEM method

Figure 4.4: The three-beam airplane wing with coordinate system and geometry

Figure 4.5: The FEM convergence results for three-beam wing

Figure 4.6: The dually coupled bending-torsion coupled beam cross-sectional geometry

Figure 4.7: The first eight modes of the $W A D C S-5$ propeller blade for $\Omega=0$

Figure 4.8: The first eight mode shapes of the $W A D C S-5$ propeller blade for $\Omega=1567 \mathrm{rpm}$

Figure 4.9: The first eight mode shapes of the $W A D C S-5$ propeller blade for $\Omega=5884 \mathrm{rpm}$

Figure 4.10: The FEM convergence results for non-rotating triply coupled beam

Figure 4.11: The effect of rotary speed on frequencies and modes of triply coupled rotating blade

Figure 5.1: The beam bending shape functions calculated at its first four natural frequencies

Figure 5.2: The first three out-of-plane modes of vibrations for a cantilever beam based on DFE method

Figure 5.3: The DFE method convergence results for coupled bending-torsion vibrations

Figure 5.4: The comparison between the DFE and FEM convergence for coupled bending-torsion free vibrations 
Figure 5.5: The DFE results for the first five modes of coupled bending-torsion vibrations for a cantilever beam; flap (bending) displacements

Figure 5.6: The DFE results for the first five modes of coupled bending-torsion vibrations for a cantilever beam; torsional displacements

Figure 5.7: The DFE convergence results for axially loaded cantilever coupled beam $(\mathrm{T}=-1790 \mathrm{~N})$

Figure 5.8: The effect of axial force on natural frequencies of a clamped-free beam bendingbending vibrations

Figure 5.9: The DFE convergence results for bending-bending vibrations of a rotating beam

Figure 5.10: The effect of rotating speed on natural frequencies for clamped-free beam bendingbending vibrations

Figure 5.11: The first eight mode shapes of bending-bending vibrations of a clamped-free rotating beam

Figure 5.12: The comparison between the DFE and FEM convergence for non-rotating triply coupled beam

Figure 5.13: The first three modes of vibrations of non-rotating triply coupled beam

Figure 5.14: The DFE convergence results for rotating triply coupled beam

Figure 5.15: The modes of vibrations for a triply coupled rotating beam

Figure 5.16: The model for piecewise uniform beam

Figure B.1: The Algorithm of main program of FEM method for calculation of natural frequencies

Figure B.2: The Algorithm of the program which calculates the mode shapes, used for the FEM method

Figure B.3: The main DFE function bisection algorithm

Figure B.4: The algorithm of calculation of $J_{0}(\omega)$ 


\section{NOMENCLATURE:}

\begin{tabular}{|c|c|}
\hline$\left\{a_{w}\right\},\left\{a_{v}\right\},\left\{a_{t}\right\}$ & Non-nodal parameters \\
\hline$c_{w}, c_{v}, c_{\phi}, c_{T}$ & Constant parameters in uncoupled stiffness matrix \\
\hline$D_{f, w}, D_{f, v}$ & Denominators of element stiffness matrices \\
\hline$D E V$ & Deviator matrix \\
\hline$e$ & Off set beam root \\
\hline$e_{I}$ & Eccentricity between elastic center and center of mass \\
\hline$E$ & Modulus of elasticity \\
\hline$\{F\}$ & Perturbed force \\
\hline$G J$ & Torsion rigidity \\
\hline$I_{y}, I_{z}, I_{z y}, I_{a}$ & Mass moments of inertia \\
\hline$j, j_{0}, j_{m}, j_{t}, j_{f}$ & Parameters used in Wittrick-Williams method \\
\hline$J$ & Jacobian \\
\hline$K^{e}, K_{1}^{e}, K_{2}^{e}, K_{3}^{e}$ & Element stiffness matrices \\
\hline$K$ & Beam stiffness matrix \\
\hline$K(\omega)$ & Dynamic (frequency dependent) stiffness matrix \\
\hline$L$ & Length of beam \\
\hline$l_{e}, l$ & Element length \\
\hline$m$ & Mass per unit length \\
\hline$M^{e}$ & Element mass matrix \\
\hline$M$ & Beam mass matrix \\
\hline$M_{x}$ & Torque along $x$ axis \\
\hline$M_{y}, M_{z}$ & Bending moments \\
\hline$N_{B-w}$ & Shape function for bending in $z$ direction \\
\hline$N_{B-v}$ & Shape function for bending in y direction \\
\hline$N_{T-\phi}$ & Shape function for torsion along $x$ axis \\
\hline$<P(\xi)>_{f},\left\langle P(\xi)>_{t}\right.$ & Vectors of basis functions for flexural and torsional displacements \\
\hline$s[K(\omega)]$ & Sign count of matrix $K(\omega)$ \\
\hline$T, T(x)$ & Axial load / Centrifugal force \\
\hline$\{u\}$ & Nodal displacement \\
\hline$\left\{u_{n}\right\}$ & Element nodal displacement \\
\hline$v$ & Cord-wise displacement (in y direction) \\
\hline$V_{y}, V_{z}$ & Shear forces \\
\hline
\end{tabular}


$w$

$W$

$W_{\text {int }}$

$W_{\text {ext }}$

$W_{f, w}, W_{f, v}, W_{t, \phi}$

$x$

$\kappa_{m l}, \kappa_{m 2}, \kappa_{m}$

$\delta u, \delta w, \delta v, \delta \phi$

$\alpha, \beta$

$\phi$

$\theta$

$\tau$

$\omega, \omega_{i}, \omega^{*}$

$\omega^{*}$

$\omega_{l}$,

$\omega_{u}$

$\Omega$

$\xi$

Flap-wise displacement (in $z$ direction)

Total virtual work

Virtual work done by internal forces (stresses)

Virtual work done by external forces

Virtual work of flexural and torsion displacements

Physical coordinate

Radius of gyration

Virtual displacements

Element frequency dependent parameters for bending vibrations

Torsion displacement

Pre-twisting angle

Element frequency dependent parameter for torsional vibrations

Natural frequencies

Trial natural frequency

Lower natural frequency

Upper natural frequency

Angular (rotating) velocity of beam

Element coordinate 


\section{CHAPTER 1： INTRODUCTION}

\subsection{Introduction}

The study of the dynamic behaviour of flexible structures is an intrinsic part of design of such systems. The determination of dynamic characteristics of structures is very important in many terrestrial engineering applications, such as automotive industries, industrial robots design and automations, buildings and bridges, and aerospace structural problems such as fixed and rotary aircraft wings and structures, control surfaces, satellite antenna and solar and many more simple or complicated systems. In many cases, when there is a source of vibration with variable frequency, the vibration behaviour of a system is studied to avoid resonance in transient response, where design engineer is to find the frequency spectrum of the system and check the interference of working frequencies and the system natural frequencies.

Besides, in many structural problems, the system modal analysis is also important, especially when the design is subject to geometric considerations. For example, the nodes locations in the natural modes could be used to define the supports. Also, the changes in the boundary conditions can considerably affect the system's natural frequencies.

Beams vibration analysis plays a significant role in the investigation of the dynamic behaviour of flexible structures, since many systems can be simply represented by a beam model. Bridges, slender propellers blades, airplane wings and satellite antennas are modeled, at least for the first few natural frequencies, as beam structures. Therefore, many research works have been carried out on the new analytical and numerical methods for beam vibrations with different mechanical and geometrical characteristics [1-13,15-25,27-30].

Beams exhibit different vibrational behaviours depending on their mechanical and geometrical properties. A beam can undergo single or multiple bending (lateral) vibrations, torsional vibrations, longitudinal vibrations and/or a combination of some or all displacements. For the coupled systems, the differential equations governing the structural vibrations are coupled. The coupled equations are widely used in modeling and analysis of aeronautical systems, namely the aircraft wings and stator and rotary 
compressor and turbine blades, etc. exhibiting coupled bending-bending, bending-torsion or bending-bending-torsion vibrations.

Houbolt and brooks (1956) [1] investigated the beam coupled vibrations by formulating a rotating beam model with coupling between flap-wise bending, cord-wise bending and torsion, where the beam has variable cross-sectional properties, twist angle and is subjected to external aerodynamic lift and drag forces. The Euler-Bernoulli bending and St. Venant torsion beam theories were considered where the shear deformation and rotary inertia effects are neglected.

Computers opened a new horizon to engineering problems and many researchers then focused their efforts to develop numerical methods where the exact analytical solutions to the system's governing equations are not known. Murthy (1976) [2] used the Transmission Matrix Method (TMM) to solve the triply coupled differential equations governing the vibrations of twisted non-uniform blades. The state vector (satisfying the differential equations) and backward transmission matrix were employed to form the system's eigenproblem. The eigenproblem was then solved to obtain natural frequencies and mode shapes of the blade. In another attempt, Murthy used the Integrating Matrix Method (IMM) to solve the bending-torsion coupled equations of rotating beams [3,4], where equations are written in matrix form and are integrated to develop the system eigenproblem.

Lang and Nemat-Nasser (1979) [5] proposed a new quotient method to investigate the out-of-plane, in-plane and torsional vibrations of pre-twisted non-uniform blades. This method is based on a variational statement of equations and can be used to find the blade's natural frequencies and mode shapes.

Magari et al. (1987) [6] introduced a Finite Element Method (FEM) and the Hermite bending shape functions were employed to solve the equations of motion for triply coupled beam vibrations. The resulting equations were then solved using MSC/NASTRAN program. The development of numerical methods was continued by Surace et al. $[16,17]$ and they investigated the bending-bending-torsion coupled beam vibrations using the integral formulation based on Green functions (structural influence functions). 
Besides the numerical approaches, there have also been some attempts to find the analytical solutions of the differential equations governing the coupled beam dynamics. Based on the Dynamic Stiffness Matrix (DSM) method, Banerjee introduced the exact solutions for different coupled bending-torsion beam vibrations [7-13] where the Wittrick-Williams (W-W) root counting algorithm $[14,15]$ was used to find the system's natural frequencies. The exact DSM solution is limited to simple problems and it cannot be used to solve the complex beam geometries and configurations.

Furthermore, many researches have also been focused on more advanced beam theories to incorporate the effect of warping, shear deformation and rotary inertia, etc. Arpaci et al. [18] introduced an exact solution method for thin-walled open cross section beams considering warping and rotary inertia effects. Subrahmanyam et al. [19] presented an approach for beam vibrations analysis including the shear deformation, rotary inertia, warping and thermal effects. This model can be used for turbine blades where the beam does not satisfy Euler-Bernoulli assumptions and the thermal effects must be taken into account [19]. The effect of warping on the axially loaded coupled bending-torsion beam was investigated by Jun et al. [20]. As shown in this research, ignoring the warping effect causes decrements in natural frequencies. Also, for higher modes, the warping effect is more pronounced.

The Dynamic Finite Element (DFE) method, first introduced by Hashemi (and his coauthors) [21-25], represents an intermediate method between analytical exact DSM and numerical FEM methods. In this approach, the Dynamic (frequency dependent) Trigonometric Shape Functions (DTSF's), employed as approximation functions, are formulated based on the solution of beam uncoupled governing differential equations. Similar to the DSM method, the DFE produces a non-linear eigenvalue problem and the Wittrick-Williams algorithm $[14,15,26]$ can therefore be used to extract the natural frequencies of the system. The DFE has been applied to the uncoupled flexural and bending-torsion vibrations of uniform and non-uniform beams where the equations of motion are geometrically and/or materially coupled, resulting in excellent convergence rates [21-25].

It has been proven that the DFE combines the advantages of the FEM and DSM methods and results in an accurate, flexible, and systematic method to determine the 
natural frequencies of beams and beam-like structures. The DFE can be advantageously used for preliminary design of mechanical systems made of flexible beam assemblies, where designer needs to portrait the general dynamic behaviour of the system with acceptable accuracy before starting the detailed analysis and design. Complete modeling of complex systems takes relatively long time and is expensive, and employing the DFE especially for systems with repeated sub-structures can provides a versatile tool to depict the dynamic characteristics of the system.

\subsection{Objective}

The objective of this thesis is to develop and validate a new DFE formulation for triply coupled bending-bending-torsion vibrations of rotating and non-rotating beams. A classical FEM approach is also presented and the two methods are compared. Both DFE and FEM formulations are developed based on Galerkin-type weighted residual method (WRM). Due to the nature of the DFE method, the resulting eigenproblem is nonlinear. A dedicated Wittrick-Williams root counting algorithm $[14,15,26]$ which provides a powerful tool to evaluate the natural frequencies of nonlinear eigenproblems is introduced. The method can then be implemented to investigate the natural frequencies and corresponding modes of free vibrations of dually and triply coupled beams. The method presented in this research is able to calculate the $i^{\text {th }}$ eigenvalue (natural frequency) of the resulting nonlinear eigenproblem.

This research also investigates the coupled vibrations of flexible beams and the effect of various geometric and dynamic parameters on the system behaviour. The dynamic coupling (caused by rotating speed and/or constant axial load) and geometrical coupling (caused by pre-twist angle and/or distance between mass and elastic center) are studied by several illustrative examples and the natural frequencies and modes of free vibrations of beams and rotating blades are evaluated. These examples provide a general understanding and a better insight to the free vibrations of beams and blades.

The DFE and FEM formulations presented in this research can be used to evaluate the fundamental natural frequencies and the corresponding modes of a beam structure. In 
many engineering design problems, the designer needs to get a general idea about the dynamic behaviour of the system even before a detailed and rigorous FEM analysis starts. In such cases, having a reliable, simple and accurate simulation tool is helpful.

\subsection{Thesis Organization}

In order to construct an accurate DFE formulation for the free vibration analysis of blades, a progressive procedure is adopted. The modeling starts with the well-known classic FEM method for beam's pure bending vibration and finally comes to an end with a DFE model for coupled bending-bending-torsion vibrations of rotating blades.

In Chapter 1, the importance of mechanical vibrations and the vibration analysis of flexible structures, in general, and the coupled beam vibrations, in particular, are briefly discussed. Some of the numerical methods used in analysis of rotating and nonrotating beams are reviewed, and the DFE as a bridge between exact solution method (DSM) and finite element method (FEM) is outlined.

In Chapter 2, the equations of motions for the coupled flap-wise, cord-wise and torsional vibrations of rotating beams along with the boundary conditions are presented. The Euler-Bernoulli bending and St. Venant torsion beam theories are considered where the shear deformation, rotary inertia and warping are neglected. The equations are coupled due to the geometrical and mechanical properties and the dynamics of the beam. Then equations of motion and the boundary conditions for coupled bending-torsion and bending-bending vibrations are extracted from the triply coupled equations.

In Chapter 3, a dedicated Wittrick-Williams root counting algorithm for calculation of natural frequencies of a flexible structure is discussed. The solution methodology is explained for the non-linear eigenvalue problems resulting from the frequency dependent element dynamic stiffness matrices. As it is then briefly discussed, the corresponding modes of coupled vibration can be extracted using a perturbation technique.

In Chapter 4, the Galerkin based finite element methodology is described and the coupled equations of motion along with static Hermite polynomial shape functions are 
used to derive the FEM method. The FEM approach then is applied to the triply coupled beams as well as coupled bending-torsion and bending-bending beams to verify the solution method.

In Chapter 5, the Dynamic Finite Element (DFE) formulation is introduced. The Dynamic Trigonometric Shape Functions (DTSF's) are used to form the frequency dependent stiffness matrix. The Wittrick-Williams root counting method is then employed to find the natural frequencies. Some illustrative examples of dually and triply coupled beams are then discussed to prove the validity of the method.

In concluding Chapter 6, the formulations introduced in this research work are reviewed. A comparative study between DFE and FEM methods is then provided, where the advantages of each method are highlighted. At the end, the direction and future of the research is stated.

In appendix $\mathrm{A}$, the solutions of uncoupled bending and torsion vibrations of a cantilever beam are derived. The solutions are used to develop Dynamic Trigonometric Shape Functions for DFE method.

Appendix B introduces the program logic and the algorithm for FEM and DFE methods, and explains the differences between two programs. Also the functions which have been developed and used in the FEM and DEF programs are discussed. 


\section{CHAPTER 2: EQUATIONS OF MOTION GOVERNING COUPLED VIBRATIONS OF ROTATING BEAMS}

\subsection{Introduction}

The governing differential equations of motion for coupled vibrations of rotating beams incorporating different levels of complexity have been introduced in several occasions $[1,16,22,23,25]$. The proposed models have been developed using different methods and consist of one or more geometric/dynamic parameters leading the coupling between equations of motion. Regardless of the derivation method, the resulting general equations can be easily reduced to special cases such as constant twisting angle, nonrotating beam with constant tension, rotating beam without eccentricity, coupled bendingtorsion $[2,16]$, bending-bending $[2,16,27]$ and the simple case of uncoupled bending and torsion vibrations of a beam [34].

\subsection{Model}

A cantilever rotating beam (Figure 2.1) with length $L$, and an offset $e_{l}$ from rotating axis (i.e. hub radius), distance $\boldsymbol{e}$ between mass centroid and elastic (shear) center and rigid cross-section is the basis of the model. All rigidities are assumed to be constant, or piecewise constant, along $\boldsymbol{x}$-axis. The rigidities are: flexural rigidities $\boldsymbol{E} \boldsymbol{I}_{z}, \boldsymbol{E} \boldsymbol{I}_{\boldsymbol{y}}, \boldsymbol{E I _ { z y }}$ and Torsional rigidity $\boldsymbol{G J}$. The rigidities can be determined experimentally or theoretically. The beam has a pre-twist angle $\theta$. 


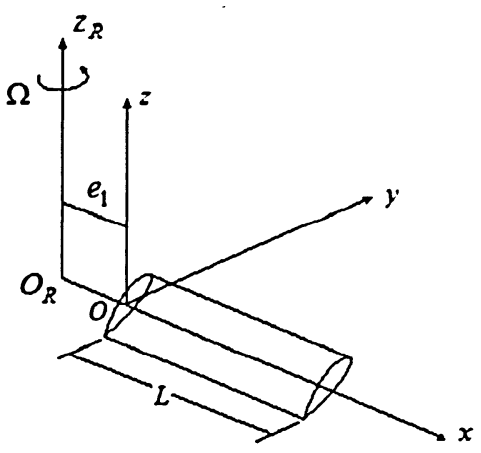

(a)

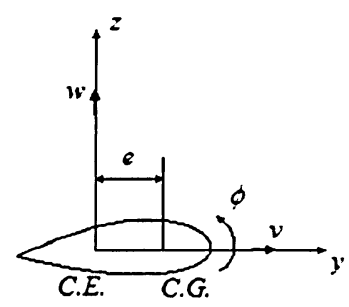

(b)

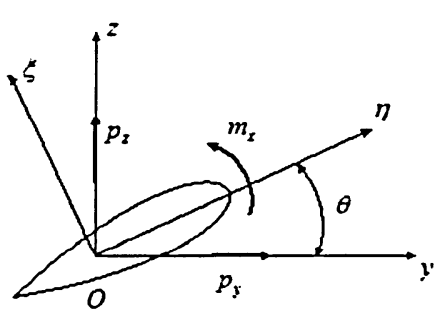

(c)

Figure 2.1: The beam configuration and geometrical parameters.

The beam undergoes three displacements: $w$ and $v$ are out-of-plane and in-plane displacements, respectively, associated with bending vibrations in two directions and $\phi$ is the rotational displacement associated with torsion.

\subsection{Assumptions}

The Euler-Bernoulli bending and St. Venant shear beam theories are exploited. The shear deformation, the rotary inertia effect, warping and thermal effects are neglected. The small linear displacements are considered, the axial displacements are neglected and the bending slope is set equal to the derivative of bending displacement with respect to spatial variable $\boldsymbol{x}$.

\subsection{Governing Differential Equations of Motion}

There have been many studies to derive the differential equations governing the free vibrations of rotating blades. Houbolt and Brooks [1] followed the stress-strain analysis in which the longitudinal strains are expressed in terms of two perpendicular bending displacements and torsional displacement. The stress is then integrated over the 
beam cross sectional area to evaluate the flap-wise, cord-wise and torsion moments at any section of the beam. The resulting internal and external moments and forces are used in Newton's second law to relate the forces with accelerations. The Newton's second law results in the final differential equations of motion. Magari et al. [6] employed the Hamilton's principle in which the action function is minimized in the time interval, and the internal potential energy and kinetic energy are calculated in terms of displacements. Using any of these two methods, the displacements are functions of time $(t)$ and $\boldsymbol{x}$ and final differential equations of motion consist of time and spatial partial derivatives. Using separation of variables technique and based on the simple harmonic motion assumption, one can get the final spatial differential equations, governing the free vibrations of rotating beams as follow:

$$
\begin{gathered}
\left(E I_{y} w^{\prime \prime}+E I_{z y} v^{\prime \prime}\right)^{\prime \prime}-\left(T w^{\prime}\right)^{\prime}-\left[\Omega^{2} e m\left(x+e_{1}\right) \phi \cos \theta\right]^{\prime} \\
-\omega^{2} m(w+e \phi \cos \theta)=0 \\
\left(E I_{z} v^{\prime \prime}+E I_{z y} w\right)^{\prime \prime}-\left(T v^{\prime}\right)^{\prime}+\left[\Omega^{2} e m\left(x+e_{1}\right) \phi \sin \theta\right]^{\prime} \\
+\Omega^{2} e m \phi \sin \theta-\omega^{2} m(v-e \phi \sin \theta)-\Omega^{2} m v=0 \\
-\left(G J \phi^{\prime}\right)^{\prime}-\Omega^{2} e m\left(x+e_{1}\right)\left(v^{\prime} \sin \theta-w^{\prime} \cos \theta\right) \\
+\Omega^{2} e m v \sin \theta+\Omega^{2} m\left(\kappa_{m 2}^{2}-\kappa_{m 1}^{2}\right) \phi \cos 2 \theta \\
-\omega^{2} m \kappa_{m}^{2} \phi+\omega^{2} e m(v \sin \theta-w \cos \theta)=0
\end{gathered}
$$

where " ' " represents the differentiation with respect to $x, \omega^{2}$ is a result of two times differentiation with respect to time, $\boldsymbol{\Omega}$ is the angular (rotating) velocity of the beam about $z$-axis, $\boldsymbol{m}$ is the mass per unit length of the beam and $T$, the tensile axial force acting on the beam, is the centrifugal force and is calculated at any point of beam from:

$$
T(x)=\int_{x}^{L} \Omega^{2} m\left(x+e_{1}\right) d x
$$


One can see that the differential equations (2.1) to (2.3) are coupled due to the geometric parameters $\boldsymbol{e}$ and $\boldsymbol{\theta}$, and the dynamic parameters $\boldsymbol{\Omega}$.

\subsection{Boundary Conditions}

For free vibrations of a cantilever beam, all displacements at the clamped end, and the internal forces and moments at free end are equal to zero. The boundary conditions for a clamped-free beam are:

$$
\begin{array}{lc}
\text { At } \boldsymbol{x}=0: & v=w=\phi=v^{\prime}=w^{\prime}=0 \\
\text { And at } \boldsymbol{x}=\boldsymbol{L}: & M_{x}=M_{y}=M_{z}=V_{y}=V_{z}=0
\end{array}
$$

where $\boldsymbol{M}_{\boldsymbol{x}}, \boldsymbol{M}_{\boldsymbol{y}}$ and $\boldsymbol{M}_{\boldsymbol{z}}$ are internal moments about $\boldsymbol{x}, \boldsymbol{y}$ and $\boldsymbol{z}$ axes, respectively, and $\boldsymbol{V}_{\boldsymbol{y}}$ and $V_{z}$ represent internal shear forces in $y$ and $z$ directions.

For uncoupled beam equations, shear forces and moments expressions are reported in any solid mechanics textbook, but for coupled vibrations, shear forces and moments are to be calculated considering the coupling terms. The internal forces and moments at any point along the beam length are then calculated from following equations [1]:

$$
\begin{gathered}
V_{z}=-\left(E I_{y} w^{\prime \prime}+E I_{z y} v^{\prime \prime}\right)^{\prime}+T w^{\prime}+\left(\Omega^{2} e m\left(x+e_{1}\right) \phi \cos \theta\right) \\
V_{y}=-\left(E I_{z y} w^{\prime \prime}+E I_{z} v^{\prime \prime}\right)^{\prime}+T v^{\prime}-\left(\Omega^{2} e m\left(x+e_{1}\right) \phi \sin \theta\right) \\
M_{x}=G J \phi^{\prime} \\
M_{y}=E I_{y} w^{\prime \prime}+E I_{z y} v^{\prime \prime} \\
M_{z}=E I_{z} v^{\prime \prime}+E I_{z y} w^{\prime \prime}
\end{gathered}
$$


The equations (2.1) to (2.3) and the boundary conditions (2.5) to (2.11) fully define the dynamic behaviour of flexible linear rotating beams and are exploited in next chapters to find the natural frequencies and mode shapes of the triply coupled beams vibrations.

\subsection{Equations of Motion for Dually Coupled Vibrations}

As already stated, the triply coupled differential equations of beam vibrations (2.1) to (2.3) and the boundary conditions (2.5) to (2.11) can be used to derive the differential equations of motions for dually coupled vibrations of a beam.

\subsubsection{Coupled Bending-Bending Vibrations}

The coupled flap-wise and cord-wise bending vibrations of a rotating beam are governed by the following differential equations:

$$
\begin{gathered}
\left(E I_{y} w^{\prime \prime}+E I_{z y} v^{\prime \prime}\right)^{\prime \prime}-\left(T w^{\prime}\right)^{\prime}-\omega^{2} m w=0 \\
\left(E I_{z} v^{\prime \prime}+E I_{z y} w\right)^{\prime \prime}-\left(T v^{\prime}\right)^{\prime}-\omega^{2} m v-\Omega^{2} m v=0
\end{gathered}
$$

Obtained by setting $\varphi$ to zero in equations (2.1) and (2.2). The boundary conditions at free end, in this case, reduce to following form:

$$
\begin{gathered}
V_{z}=-\left(E I_{y} w^{\prime \prime}+E I_{z y} v^{\prime \prime}\right)^{\prime}+T w^{\prime}=0 \\
V_{y}=-\left(E I_{z y} w^{\prime \prime}+E I_{z} v^{\prime \prime}\right)^{\prime}+T v^{\prime}=0 \\
M_{y}=E I_{y} w^{\prime \prime}+E I_{z y} v^{\prime \prime}=0
\end{gathered}
$$




$$
M_{z}=E I_{z} v^{n}+E I_{z y} w^{\prime \prime}=0
$$

One can see that the angular velocity of the beam, $\mathbf{\Omega}$, doesn't have any contribution in coupling terms and only appears in the expression for centrifugal force (see equation 2.9), $T$, and the coupling between two in-plane and out-of-plane of rotation lateral displacements is related to asymmetric term $\boldsymbol{E} \boldsymbol{I}_{z \boldsymbol{y}}$. Also, the eccentricity $\boldsymbol{e}$ disappears when torsional displacement $\phi$ vanishes. It means that the eccentricity between centroid and elastic center doesn't affect the bending-bending coupled equations.

It is also to be noted that out-of-plane flap vibrations are only affected by centrifugal effect $\left(T w^{\prime}\right)^{\prime}$, whereas the in-plane displacement is related to the centripetal term, $-\mathbf{\Omega}^{\mathbf{2}} \boldsymbol{m} \boldsymbol{v}$, as well. The centrifugal term increases the beam stiffness in both directions, while the centripetal term, due to the negative sign, decreases the stiffness in in-plane direction and causes decrement in cord-wise mode frequencies.

\subsubsection{Coupled Bending-Torsion Vibrations}

The differential equations governing the coupled bending-torsion vibrations can be extracted from the triply coupled general case by setting $v=0$ :

$$
\begin{gathered}
\left(E I_{y} w^{\prime \prime}\right)^{n}-\left(T w^{\prime}\right)^{\prime}-\left[\Omega^{2} e m\left(x+e_{1}\right) \phi \cos \theta\right]^{\prime} \\
-\omega^{2} m(w+e \phi \cos \theta)=0 \\
-\left(G J \phi^{\prime}\right)^{\prime}+\Omega^{2} e m\left(x+e_{1}\right) w^{\prime} \cos \theta-\omega^{2} e m w \cos \theta \\
+\Omega^{2} m\left(\kappa_{m 2}^{2}-\kappa_{m 1}^{2}\right) \phi \cos 2 \theta-\omega^{2} m \kappa_{m}^{2} \phi=0
\end{gathered}
$$

where, in this case, the boundary conditions at free end are: 


$$
\begin{gathered}
V_{z}=-\left(E I_{y} w^{\prime \prime}\right)^{\prime}+T w^{\prime}+\left(\Omega^{2} e m\left(x+e_{1}\right) \phi \cos \theta\right)=0 \\
M_{x}=G J \phi^{\prime}=0 \\
M_{y}=E I_{y} w^{\prime \prime}=0
\end{gathered}
$$

The coupling occurs because of non-coincident shear and mass axes $(e \neq 0)$ and the beam rotating speed $\boldsymbol{\Omega}$. Even though the pre-twist angle $\theta$ appears in coupling term, but it doesn't have direct contribution in these terms. In words, if $\theta$ vanishes, the nature of equations will not change.

In this chapter the differential equations of motion governing the triply and dually coupled vibrations of rotating beams were presented. In Chapter 3, the Wittrick-Williams root counting method, used to evaluate the natural frequencies of an eigenproblem, will be presented. Then, in Chapter 4 and 5 , the governing differential equations of motion introduced in this chapter are solved, using the FEM and DFE methods, respectively, to evaluate the natural frequencies and modes of rotating and non-rotating beams. 


\section{CHAPTER 3: SOLUTION OF EIGENPROBLEMS}

\subsection{Introduction}

Many engineering problems, such as buckling and vibration analyses of flexible structures, lead to one of the following equations:

$$
\begin{gathered}
{[A]\{x\}=\lambda[B]\{x\}} \\
{[A]\{x\}=\lambda\{x\}} \\
{[A(\lambda)]\{x\}=0}
\end{gathered}
$$

known as eigenvalue problems or simply eigenproblems. Here $[A],[B]$ and $[A(\lambda)]$ are $\boldsymbol{n} \times \boldsymbol{n}$ matrices; $\{\boldsymbol{x}\}$ is an $\boldsymbol{n}$ dimensional vector; $\{\boldsymbol{x}\}$ and $\boldsymbol{\lambda}$ are eigenvector and eigenvalue, respectively. When $[A]$ and $[B]$ are constant, the above equation is called a linear eigenproblem (see (3.1) and (3.2)), whereas $\lambda$-dependent components lead to a nonlinear eigenproblem (3.3). In vibration analysis of flexible structures, the eigenvalues and eigenvectors are equivalent to natural frequencies and mode shapes, respectively, where $\lambda=\omega^{2}$.

There exist several well-known iterative numerical algorithms established to find the eigenvalues and eigenvectors of the linear eigenproblems. The approach introduced in this chapter, developed by Wittrick and Williams [14, 15, 27], provides a powerful and robust tool for, but is not limited to, nonlinear eigenproblems of form (3.3), where the coefficient matrix $[A(\omega)]$ is frequency dependent and other algorithms may skip some natural frequencies. 


\subsection{Wittrick-Williams (W-W) Method}

As already stated, both the DSM and DFE methods lead to a nonlinear eigenproblem due to the fact that the approximation functions and the element stiffness matrices are frequency dependent. In this case, the coefficient matrix in (3.3) is the nonlinear frequency dependent dynamic stiffness matrix, $[A(\lambda)]=[K(\omega)]$. The WittrickWilliams (W-W) root counting algorithm $[14,15]$ can then be exploited to find all natural frequencies lying below a trial frequency $\omega^{*}$. The advantage of W-W approach is that for the periodic structures and systems composed of sub-structures, the natural frequencies of sub-structures and poles of the system can also be calculated. Besides, the constrained nodes and special boundary conditions which produce repeated frequencies can also be studied [15].

\subsubsection{Theory}

For a flexible structure, there are two possible solutions pertaining to the equation $\left[K_{n}(\omega)\right]\left\{u_{n}\right\}=0:$

a) $\left|\left[K_{n}(\omega)\right]\right|=0$, where $\left\{u_{n}\right\} \neq 0$ is one set of solution,

b) $\left|\left[K_{n}(\omega)\right]\right|=\infty$, where $\left\{\boldsymbol{u}_{n}\right\}=0$ corresponds to the case where displacements $\left\{\boldsymbol{u}_{\boldsymbol{n}}\right\}$ are 'constrained nodes' or nodes in the natural modes of vibrations.

Suppose that $\omega$ denotes the natural frequency of the structure. Then, it is known that $j$, the number of natural frequencies of the system between zero and $\omega^{*}$, as $\omega$ increases, is given by:

$$
j=j_{0}+s\left(\left[K\left(\omega^{*}\right)\right]\right)
$$


where $\left[K\left(\omega^{*}\right)\right]$ is the overall dynamic (frequency dependent) stiffness matrix evaluated at $\omega=\omega^{*}$, and $s\left(\left[K\left(\omega^{*}\right)\right]\right)$ is the number of negative elements on the leading diagonal of $\left[K\left(\omega^{\star}\right)\right]^{\Delta}$ and $\left[K\left(\omega^{\star}\right)\right]^{\Delta}$ is the upper triangular matrix obtained by applying the standard Gauss elimination method to the $[K(\omega)]$ with no column interchange. $j o$ is the number of natural frequencies of the structure still lying between $\omega=0$ and $\omega=\omega^{*}$ when displacement components to which $[K(\omega)]$ corresponds are all zero (in such a case, the beam can still have natural frequencies when all its nodes are clamped, because the formulation allows each individual (beam) element to have an infinite number of degrees of freedom between nodes). Thus:

$$
j_{0}=\sum_{m=1}^{N E} j_{m}
$$

where $j_{m}$ is the number of natural frequencies between $\omega=0$ and $\omega=\omega^{*}$ for an element with its ends clamped, while the summation extends over all elements.

It can be shown [14] that $j_{m}$ for the case of coupled bending-bending-torsion vibrations is calculated as:

$$
j_{m}=j_{t}+j_{f, w}+j_{f, v}
$$

where:

$$
\begin{gathered}
j_{t}=\text { the largest integer }<\tau / \pi \\
j_{f, w}=i_{w}-\frac{1}{2}\left[1+(-1)^{i_{w}} \operatorname{sgn}\left(D_{f, w}\right)\right] \\
i_{w}=\text { the largest integer }<\alpha_{w} / \pi
\end{gathered}
$$




$$
\begin{gathered}
j_{f, v}=i_{v}-\frac{1}{2}\left[1+(-1)^{i_{v}} \operatorname{sgn}\left(D_{f, v}\right)\right] \\
i_{v}=\text { the largest integer }<\alpha_{v} / \pi
\end{gathered}
$$

$D_{f, v}$ and $D_{f, w}$ are the denominators of the element stiffness matrix for in-plane, $v$, and outof-plane, $w$, flexural displacements, respectively and $\alpha$ and $\tau$ are element frequency dependent parameters [21].

Thus, by exploiting the equations (3.4) to (3.11), it is possible to converge on any required natural frequencies, given the fact that the expressions for the dynamic stiffness matrix and the clamped-clamped natural frequencies are known.

\subsubsection{Application to the Beam Vibrations}

A beam with uncoupled out-of-plane vibrations is used to demonstrate WittrickWilliams method. The equation of motion for a cantilever beam with out of plane flexural vibrations is:

$$
\left(E I_{y} w^{n}\right)^{n}-\omega^{2} m w=0
$$

After applying the natural (force) boundary conditions, using the DFE or DSM method [21], the final eigenproblem can be written as:

$$
[K(\omega)]\{u\}=0
$$

For a one-element beam model, the natural frequencies are obtained when the determinant of stiffness matrix, $[K(\omega)]$, vanishes. In other words, when the $[K(\omega)]$ 
determinant is plotted versus frequency (Figure 3.1), the intersections between the curve and $\omega$-axis are the natural frequencies. It can be seen from Figure 3.1 that between the second and third natural frequencies there exist two poles, where $|[K(\omega)]| \rightarrow \infty$.

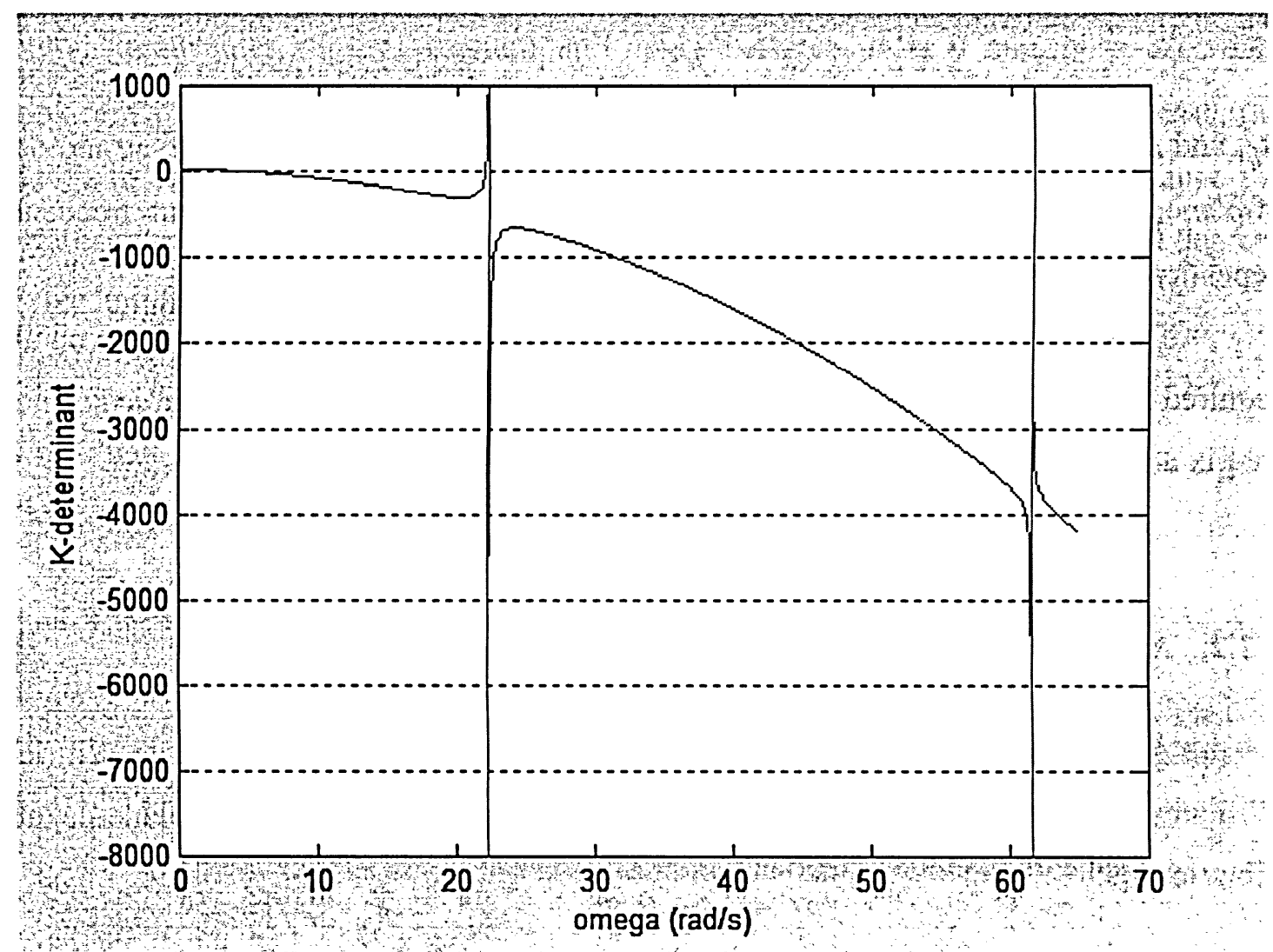

Figure 3.1: The determinant of stiffness matrix versus frequency.

Investigation of the parameters $s[K(\omega)]$ and $j_{f, w}$ shows that these numbers can change independently. The variation of sign count of stiffness matrix, $s[K(\omega)]$, for a beam with one element is shown in Figure 3.2: 


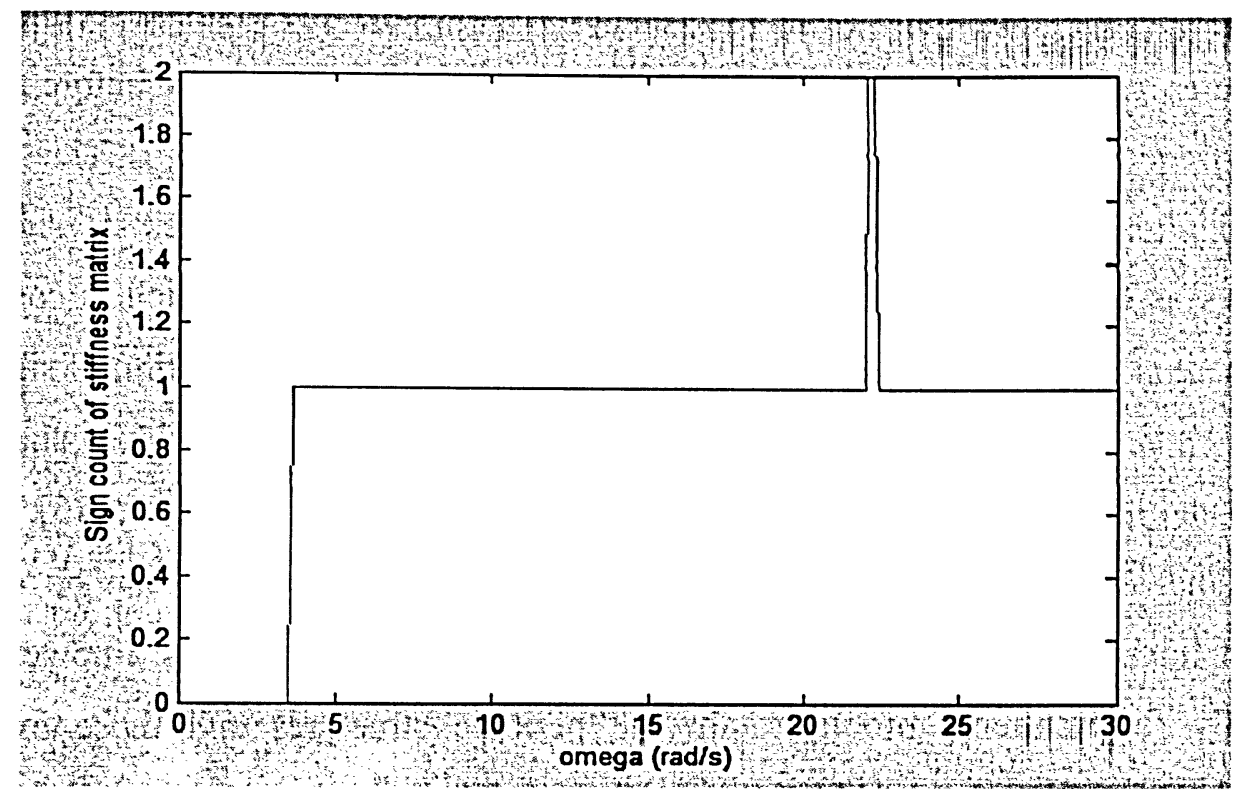

Figure 3.2: The sign count of stiffness matrix versus frequency.

and $j_{f, w}$ varies as shown in Figure 3.3:

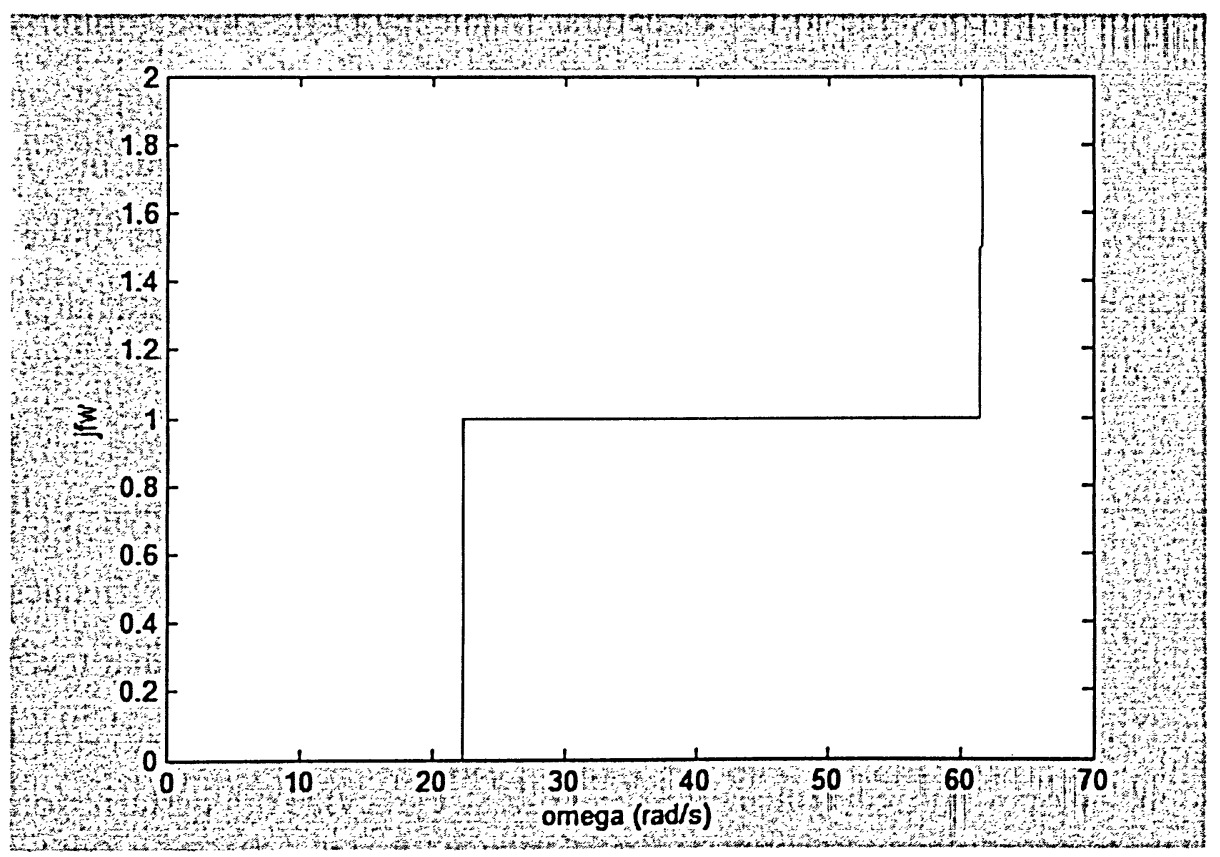

Figure 3.3: The variation of $j_{f, w}$ versus frequency. 
Closer look at Figures (3.1), (3.2) and (3.3) shows that the first and second natural frequencies can be obtained by checking the sign count, that means before the first natural frequency, the sign count is zero and at $\omega=\omega_{1}$ it jumps to 1 . This jump is repeated after the second natural frequency $\omega_{2}$, but immediately after $\omega_{2}$ sign count decreases as one unit, and this decrement is compensated by $j_{f, w}$ (which is equal to $j_{0}$ when there is only one bending element) which jumps to 1 . The summation of sign count and $j_{f, w}$ however remain equal to 2 . It means that regardless of individual changes of $s[K(\omega)]$ and $j_{0}$, the total $j$ changes only at each natural frequency by one unit [15].

The Wittrick-Williams method, demonstrated in this section, can be used for cases of linear and/or non-linear eigenproblems. This method provides a powerful tool especially for nonlinear problems, for the periodic structures and those exhibiting repeated natural frequencies [15].

\subsection{Solution of the Eigenproblem}

By implementing the Wittrick-Williams approach, a simple bisection algorithm can be employed to converge to the $i^{\text {th }}$ natural frequency [14]. The algorithm has been introduced in Appendix B and starts with two arbitrary initial upper and lower frequencies, $\omega_{u}$ and $\omega_{l}$. A trial frequency $\omega^{*}$ then is selected and the $j$ number, corresponding to the $i^{\text {th }}$ natural frequency, is calculated. The $\omega^{*}$ changes in the way that the difference between $\omega_{u}$ and $\omega_{l}$ decreases and they converge with any desirable accuracy to the $i^{\text {th }}$ natural frequency $\omega_{i}$.

Once the natural frequencies are known, the corresponding desired mode shapes can then be calculated by perturbation method. By definition, the dynamic stiffness matrix cannot be inverted due to its zero determinant at any natural frequencies. To obtain a non-trivial solution, the frequency variable is manipulated so that the frequency dependent stiffness matrix is altered slightly (in this case $10^{-10}$ is considered as perturbation factor which guarantees the desirable accuracy). This perturbation must be small enough to prevent significant deviation in solution: 


$$
\omega^{*}{ }_{i}=\omega_{i}\left(1+10^{-10}\right)
$$

where $\omega^{*}{ }_{i}$ is the altered $i^{\text {th }}$ natural frequency, and eigenvalue equation can be written:

$$
\left[K\left(\omega^{*}{ }_{i}\right)\right]\left\{u_{i}\right\}=\{F\}
$$

where $F_{j}(j=1$ to $n)$, the components of zero force function $\{F\}$, are slightly altered from zero.

$$
F^{*}{ }_{j}=10^{-10}
$$

The eigenvectors can be evaluated by manipulating equation as:

$$
\left\{u_{i}\right\}=\left[K\left(\omega^{*} i\right)\right]^{-1}\left\{F^{*}\right\}
$$

The order of perturbation of the natural frequency, $\omega^{*}{ }_{i}$, and the force vector $\left\{F^{*}\right\}$ depends on the numerical precision. Using double precision, the $10^{\text {th }}$ order perturbation is acceptable to accurately describe the modes of deformation.

The Wittrick-Williams algorithm provides an accurate and robust tool to evaluate the natural frequencies of a nonlinear eigenproblem as well as linear eigenproblems. Using this algorithm, one can approximate any individual natural frequency and the solution can converge to the theoretical value by any desirable accuracy. This method will be used in Chapter 5, where the DFE formulation produces a nonlinear dynamic (frequency dependent) stiffness matrix. In next chapter, Chapter 4, the FEM formulations based on Galerkin-type weighted residual method will be developed to solve the governing differential equations of motion for dually and triply coupled vibrations of beams. 


\section{CHAPTER 4: FINITE ELEMENT METHOD}

\subsection{Introduction}

Finite Element Methodology (FEM) is one of the most powerful numerical methods widely used to solve the problems of complicated geometry and/or mechanical properties, where the analytical solutions are not always available. In vibration analysis of a beam there are many different approaches using the FEM (see for example $[6,17,28,29,30])$. Each of these methods transforms the differential equations of motion into an algebraic eigenvalue problem. The approach presented in this work is based on the Galerkin method and a one dimensional 2-node beam element.

\subsection{Galerkin Method}

Galerkin FEM, based on the weighted residual method, is an accurate tool with high rate of convergence used in analysis of mechanical and structural systems. Substituting the approximation functions into the differential equations results in a residual for each equation, due to the fact that the approximation functions are not the exact solutions. In Galerkin method, the approximation functions are forced to be orthogonal to the residual of equations to minimize the error, and the orthogonality conditions result in algebraic equations [31]. In what follows, the FEM formulation with application to the free vibrations analysis of triply coupled rotating beams is established.

Equations (2.1) to (2.3) can be written in following general from:

$$
\begin{aligned}
& L_{1}(w, v, \phi)=0 \\
& L_{2}(w, v, \phi)=0 \\
& L_{3}(w, v, \phi)=0
\end{aligned}
$$


where $L_{1}, L_{2}$ and $L_{3}$ are linear differential operators. As already explained, the approximation solutions, $\boldsymbol{w}^{\wedge}, v^{\wedge}$ and $\phi^{\wedge}$, result in a residual for each equation, as:

$$
\begin{aligned}
& L_{1}(\hat{w}, \hat{v}, \hat{\phi})=R_{1} \\
& L_{2}(\hat{w}, \hat{v}, \hat{\phi})=R_{2} \\
& L_{3}(\hat{w}, \hat{v}, \hat{\phi})=R_{3}
\end{aligned}
$$

and to satisfy the orthogonality conditions:

$$
\begin{aligned}
& \int_{0}^{L} \delta w\left\{L_{1}(w, v, \phi)\right\} d x=0 \\
& \int_{0}^{L} \delta v\left\{L_{2}(w, v, \phi)\right\} d x=0 \\
& \int_{0}^{L} \delta \phi\left\{L_{3}(w, v, \phi)\right\} d x=0
\end{aligned}
$$

Closer look at equations (4.3) shows that the LHS of equations represents the virtual work done by each displacement [31]:

$$
\begin{aligned}
& W_{f, w}=\int_{0}^{L} \delta w\left\{L_{1}(w, v, \phi)\right\} d x \\
& W_{f, v}=\int_{0}^{L} \delta v\left\{L_{2}(w, v, \phi)\right\} d x \\
& W_{t, \phi}=\int_{0}^{L} \delta \phi\left\{L_{3}(w, v, \phi)\right\} d x
\end{aligned}
$$

Since each integral in (4.3) vanishes, the summation of these integrals also vanishes:

$$
W_{\mathrm{int}}=W_{f, w}+W_{f, v}+W_{t, \phi}=0
$$


where in the absence of external forces (i.e., free vibrations), the total virtual work is equal to internal virtual work:

$$
W=W_{\text {int }}=0
$$

One can see that the implementation of Galerkin weak formulation also satisfies the principle of virtual work.

After substituting the equations (2.1), (2.2) and (2.3) into flexural and torsional virtual work of equations (4.4), and to satisfy the natural boundary conditions, the appropriate number of integration by parts should be applied to the terms which produce the shear forces and moments of (2.7) to (2.11):

$$
\begin{aligned}
& W_{f, w}=\int_{0}^{L}\left\{\delta w^{\prime \prime}\left(E I_{y} w^{\prime \prime}+E I_{z y} v^{\prime \prime}\right)+\delta w^{\prime}\left(T w^{\prime}\right)+\delta w^{\prime}\left[\Omega^{2} e m\left(x+e_{1}\right) \phi \cos \theta\right]\right. \\
&\left.-\delta w\left[\omega^{2} m(w+e \phi \cos \theta)\right]\right\} d x \\
&+\left[\delta w\left[\left(E I_{y} w^{\prime \prime}+E I_{z y} v^{\prime \prime}\right)^{\prime}-\left(T w^{\prime}\right)-\left(\Omega^{2} e m\left(x+e_{1}\right) \phi \cos \theta\right)\right]\right]_{0}^{L} \\
&\left.+\left[-\delta w^{\prime}\left(E I_{y} w^{\prime \prime}+E I_{z y} v^{\prime \prime}\right)\right]\right]_{0}^{L} \\
& W_{f, v}=\int_{0}^{L}\left\{\delta v^{\prime \prime}\left(E I_{z} v^{\prime \prime}+E I_{z y} w^{\prime \prime}\right)+\delta v^{\prime}\left(T v^{\prime}\right)-\delta v^{\prime}\left[\Omega^{2} e m\left(x+e_{1}\right) \phi \sin \theta\right]\right.\left.+\delta v\left[\Omega^{2} e m \phi \sin \theta-\omega^{2} m(v-e \phi \sin \theta)-\Omega^{2} m v\right]\right\} d x \\
&+\left[\delta v\left[\left(E I_{z} v^{\prime \prime}+E I_{z y} w^{\prime \prime}\right)^{\prime}-\left(T v^{\prime}\right)+\left(\Omega^{2} e m\left(x+e_{1}\right) \phi \sin \theta\right)\right]\right]_{0}^{L} \\
&+\left[-\delta v^{\prime}\left(E I_{z} v^{\prime \prime}+E I_{z y} w^{\prime \prime}\right)\right]_{0}^{L} \\
& W_{t, \phi}=\int_{0}^{L}\left\{\delta \phi^{\prime}\left(G J \phi^{\prime}\right)-\delta \phi\left[\Omega^{2} e m\left(x+e_{1}\right)\left(v^{\prime} \sin \theta-w^{\prime} \cos \theta\right)-\Omega^{2} e m v \sin \theta\right.\right. \\
&-\left.\left.\Omega^{2} m\left(\kappa_{m 2}^{2}-\kappa_{m 1}^{2}\right) \phi \cos 2 \theta+\omega^{2} m \kappa_{m}^{2} \phi-\omega^{2} e m(v \sin \theta-w \cos \theta)\right]\right\} d x \\
&+ {\left[\delta \phi\left(G J \phi^{\prime}\right)\right]_{0}^{L} }
\end{aligned}
$$

The terms produced in [ ] are the boundary terms represented by product of a displacement and its corresponding force/moment and vanish at both clamped and free 
ends. After applying the natural boundary conditions, the system equations can be discretized into element equations considering the fact that the inter-element continuity conditions must be satisfied. The equations (4.7), (4.8) and (4.9) then can be written as:

$$
\begin{aligned}
& W_{f, w}^{e}=\int_{x_{j}}^{x_{j+1}}\left[\delta w^{\prime \prime}\left(E I_{y}\right) w^{\prime \prime}+\delta w^{\prime \prime}\left(E I_{z y}\right) v^{\prime \prime}+\delta w^{\prime}(T) w^{\prime}\right. \\
&+\delta w^{\prime}\left(\Omega^{2} e m\left(x+e_{1}\right) \cos \theta\right) \phi-\delta w\left(\omega^{2} m\right) w \\
&\left.-\delta w\left(\omega^{2} e m \cos \theta\right) \phi\right] d x \\
& W_{f, v}^{e}=\int_{x_{j}}^{x_{j+1}}\left[\delta v^{\prime \prime}\left(E I_{z}\right) v^{\prime \prime}+\delta v^{\prime \prime}\left(E I_{z y}\right) w^{\prime \prime}+\delta v^{\prime}(T) v^{\prime}\right. \\
&-\delta v^{\prime}\left(\Omega^{2} e m\left(x+e_{1}\right) \sin \theta\right) \phi-\delta v\left(\Omega^{2} m\right) v \\
&+\delta v\left(\Omega^{2} e m \sin \theta\right) \phi-\delta v\left(\omega^{2} m\right) v \\
&\left.+\delta v\left(\omega^{2} e m \sin \theta\right) \phi\right] d x \\
& W_{t, \phi}^{e}=\int_{x_{j}}^{x_{j+1}} {\left[\delta \phi^{\prime}(G J) \phi^{\prime}-\delta \phi\left(\Omega^{2} e m\left(x+e_{1}\right) \sin \theta\right) v^{\prime}\right.} \\
&+\delta \phi\left(\Omega^{2} e m\left(x+e_{1}\right) \cos \theta\right) w^{\prime}+\delta \phi\left(\Omega^{2} e m \sin \theta\right) v \\
&+\delta \phi\left(\Omega^{2} m\left(\kappa_{m 2}^{2}-\kappa_{m 1}^{2}\right) \cos 2 \theta\right) \phi-\delta \phi\left(\omega^{2} m \kappa_{m}^{2}\right) \phi \\
&\left.+\delta \phi\left(\omega^{2} e m \sin \theta\right) v-\delta \phi\left(\omega^{2} e m \cos \theta\right) w\right] d x
\end{aligned}
$$

Satisfaction of inter-element continuity conditions depends on the approximation functions selected for each displacement. The element nodal variables and nodal coordinates are shown in Figure 4.1:

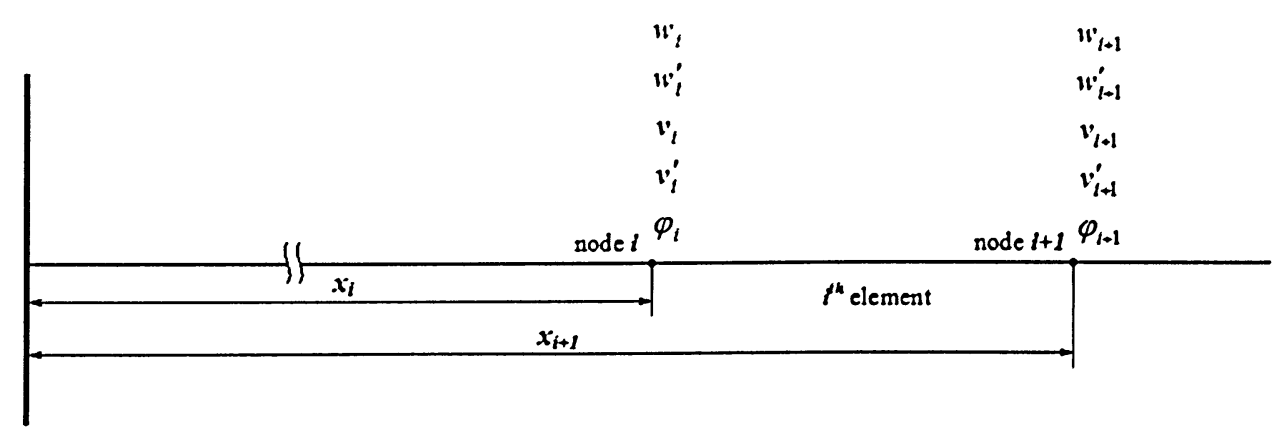

Figure 4.1: The element's nodal variables. 


\subsection{Approximation Functions}

The approximation functions are used to calculate the element displacements inside the element domain, and should satisfy the continuity conditions at the element boundary. For flexural displacements $w$ and $v$, the approximation functions should satisfy $C^{l}$ continuity, and for torsional displacement $\phi$, the function should satisfy $C^{0}$ continuity. "Hermite" cubic shape functions and linear shape functions have been developed for flexural and torsional displacements, respectively, such that they satisfy the inter-element continuity. These polynomial shape functions have been introduced in FEM text books $[32,33]$ as follow:

$$
\begin{gathered}
N_{B}=\frac{1}{4}<2-3 \xi+\xi^{3} \quad 1-\xi-\xi^{2}+\xi^{3} \quad 2+3 \xi-\xi^{3} \quad-1-\xi+\xi^{2}+\xi^{3}> \\
N_{T}=<\frac{1-\xi}{2} \quad \frac{1+\xi}{2}>
\end{gathered}
$$

where the relation between physical coordinate, $\boldsymbol{x}$, and element coordinate $\xi$, can be written as (see Figure 4.1):

$$
x(\xi)=<\frac{1-\xi}{2} \quad \frac{1+\xi}{2}>\left\{\begin{array}{c}
x_{j} \\
x_{j+1}
\end{array}\right\}
$$

and

$$
x(\xi)=\left(\frac{x_{j}+x_{j+1}}{2}\right)+\left(\frac{l}{2}\right) \xi
$$

so

$$
d x=J d \xi
$$

and

$$
J=\frac{l}{2}
$$


$J$ in equation (4.17) is called Jacobian and is taken into account where there is integration and differentiation with respect to $\xi$, and $l$ is the length of element. The transformation from the physical coordinate to the reference element coordinate is isoparametric for torsional displacement and sub-parametric for bending displacements (Figure 4.1).

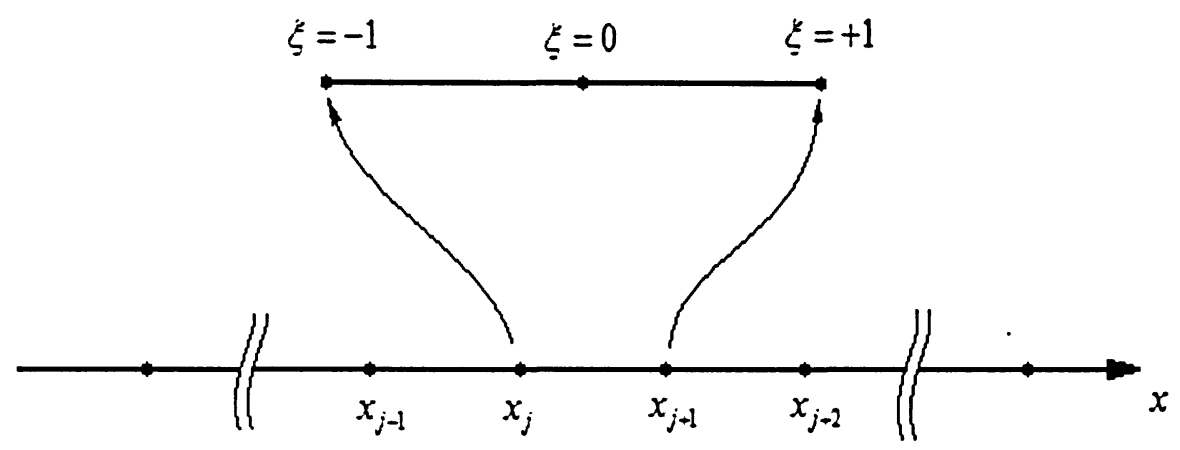

Figure 4.2: The transformation from physical coordinate to reference element coordinate.

Along the length of beam element, the displacements can be calculated by the $\boldsymbol{\xi}$ dependent shape functions as (i.e., nodal approximations):

$$
\begin{gathered}
w(\xi)=<N_{B}>_{w}\left\{\begin{array}{l}
w_{1} \\
w_{1}^{\prime} \\
w_{2} \\
w_{2}^{\prime}
\end{array}\right\}=<N_{B}>_{w}\{w\} \\
v(\xi)=<N_{B}>_{v}\left\{\begin{array}{l}
v_{1} \\
v_{1}^{\prime} \\
v_{2} \\
v_{2}^{\prime}
\end{array}\right\}=<N_{B}>_{v}\{v\}
\end{gathered}
$$




$$
\phi(\xi)=<N_{T}>\left\{\begin{array}{l}
\phi_{1} \\
\phi_{2}
\end{array}\right\}=<N_{T}>\{\phi\}
$$

where $\left\langle N_{B}\right\rangle_{w / v}$ and $\left\langle N_{T}\right\rangle$ are flexural and torsional shape functions respectively, and $\{w\},\{v\}$ and $\{\phi\}$ are nodal displacements.

\subsection{Derivation of System Eigenproblem}

Substituting virtual and element displacements by approximation functions (4.19) to (4.21) into (4.10), (4.11) and (4.12), leads to:

$$
\begin{aligned}
& W_{f, w}^{e}= \int_{-1}^{1}\left[\left(E I_{y}\right)<\delta w>\left\{N_{B}^{m}\right\}_{w}<N_{B}^{n}>_{w}\{w\}+\left(E I_{z y}\right)<\delta w>\left\{N_{B}^{n}\right\}_{w}<N_{B}^{n}>_{v}\{v\}\right. \\
&+(T)<\delta w>\left\{N_{B}^{\prime}\right\}_{w}<N_{B}^{\prime}>_{w}\{w\}+\left(\Omega^{2} e m\left(x+e_{1}\right) \cos \theta\right)<\delta w>\left\{N_{B}^{\prime}\right\}_{w}<N_{T}>\{\phi\} \\
&\left.-\left(\omega^{2} m\right)<\delta w>\left\{N_{B}\right\}_{w}<N_{B}>_{w}\{w\}-\left(\omega^{2} e m \cos \theta\right)<\delta w>\left\{N_{B}\right\}_{w}<N_{T}>\{\phi\}\right] J \mid d \xi \\
& W_{f, v}^{e}=\int_{-1}^{+1}\left[\left(E I_{z}\right)<\delta v>\left\{N_{B}^{\prime \prime}\right\}_{v}<N_{B}^{n}>_{v}\{v\}+\left(E I_{z y}\right)<\delta v>\left\{N_{B}^{\prime \prime}\right\}_{v}<N_{B}^{\prime \prime}>_{v}\{w\}\right. \\
&+(T)<\delta v>\left\{N_{B}^{\prime}\right\}_{v}<N_{B}^{\prime}>_{v}\{v\}+\left(\Omega^{2} e m \sin \theta\right)<\delta v>\left\{N_{B}\right\}_{v}<N_{T}>\{\phi\} \\
&-\left(\Omega^{2} e m\left(x+e_{1}\right) \sin \theta\right)<\delta v>\left\{N_{B}^{\prime}\right\}_{v}<N_{T}>\{\phi\}-\left(\omega^{2} m\right)<\delta v>\left\{N_{B}\right\}_{v}<N_{B}>_{v}\{v\} \\
&\left.+\left(\omega^{2} e m \sin \theta\right)<\delta v>\left\{N_{B}\right\}_{v}<N_{T}>\{\phi\}-\left(\Omega^{2} m\right)<\delta v>\left\{N_{B}\right\}_{v}<N_{B}>\{v\}\right]|J| d \xi
\end{aligned}
$$

$$
\begin{aligned}
W_{l, \phi}^{e}=\int_{-1}^{+1} & {\left[+(G J)<\delta \phi>\left\{N_{T}^{\prime}\right\}<N_{T}^{\prime}>\{\phi\}\right.} \\
& -\left(\Omega^{2} e m\left(x+e_{1}\right) \sin \theta\right)<\delta \phi>\left\{N_{T}\right\}<N_{B}^{\prime}>_{v}\left\{v^{\prime}\right\} \\
& +\left(\Omega^{2} e m\left(x+e_{1}\right) \cos \theta\right)<\delta \phi>\left\{N_{T}\right\}<N_{B}^{\prime}>_{w}\left\{w^{\prime}\right\} \\
& -\left(\Omega^{2} m\left(\kappa_{m 2}^{2}-\kappa_{m 1}^{2}\right) \cos 2 \theta\right)<\phi>\left\{N_{T}\right\}<N_{T}>\{\phi\} \\
& +\left(\omega^{2} e m \sin \theta\right)<\phi>\left\{N_{T}\right\}<N_{B}>_{v}\{v\} \\
& +\left(\Omega^{2} e m \sin \theta\right)<\delta \phi>\left\{N_{T}\right\}<N_{B}>_{v}\{v\} \\
& -\left(\omega^{2} m \kappa_{m}^{2}\right)<\phi>\left\{N_{T}\right\}<N_{T}>\{\phi\} \\
& \left.-\left(\omega^{2} e m \cos \theta\right)<\phi>\left\{N_{T}\right\}<N_{B}>_{w}\{w\}\right]|J| d \xi
\end{aligned}
$$


The products of shape functions in equations (4.22) to (4.24) produce different size matrices, i.e. $\left\{N_{B}^{\prime \prime}\right\}<N_{B}^{\prime \prime}>$ produces a $4 \times 4$ matrix, while $\left\{N_{B}\right\}<N_{T}>$ produces a $4 \times 2$ matrix. Similarly, the nodal displacements and virtual displacements are not of same size. In order to write all three equations in a unified format, the following nodal displacement vector and shape functions are introduced:

$$
\begin{aligned}
& \{u\}^{e}=<w_{1} \quad w_{1}^{\prime} \quad v_{1} \quad v_{1}^{\prime} \quad \phi_{1} \quad w_{2} \quad w_{2}^{\prime} \quad v_{2} \quad v_{2}^{\prime} \quad \phi_{2}>^{T}
\end{aligned}
$$

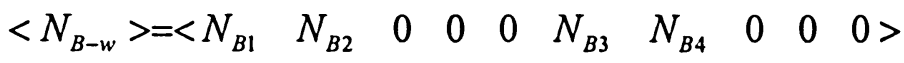

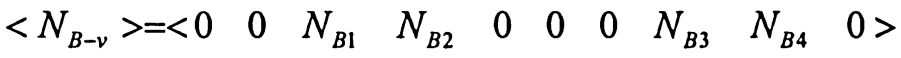

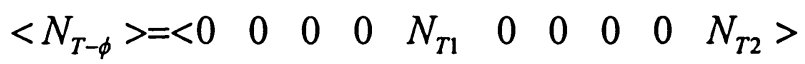

where $N_{B 1}, N_{B 2}, N_{B 3}$ and $N_{B 4}$ are the flexural shape functions and $N_{T 1}$ and $N_{T 2}$ are torsional shape functions. Using nodal parameters (4.25) to (4.28), the components of element virtual work can be written as:

$$
\begin{aligned}
& W_{f, w}^{e}=\int_{-1}^{+1}<\delta u>^{e}\left[\left(E I_{y}\right)\left\{N_{B-w}^{n}\right\}<N_{B-w}^{n}>+\left(E I_{z y}\right)\left\{N_{B-w}^{n}\right\}<N_{B-v}^{n}>\right. \\
& +(T)\left\{N_{B-w}^{\prime}\right\}<N_{B-w}^{\prime}>+\left(\Omega^{2} e m\left(x+e_{1}\right) \cos \theta\right)\left\{N_{B-w}^{\prime}\right\}<N_{T-\varphi}> \\
& \left.-\left(\omega^{2} m\right)\left\{N_{B-w}\right\}<N_{B-w}>-\left(\omega^{2} e m \cos \theta\right)\left\{N_{B-w}\right\}<N_{T-\phi}>\right]\{u\}^{e}|J| d \xi \\
& W_{f, v}^{e}=\int_{-1}^{+1}<\delta u>^{e}\left[\left(E I_{z}\right)\left\{N_{B-\nu}^{n}\right\}<N_{B-v}^{n}>+\left(E I_{z y}\right)\left\{N_{B-v}^{n}\right\}<N_{B-w}^{n}>\right. \\
& +\left(\Omega^{2} e m \sin \theta\right)\left\{N_{B-\nu}\right\}<N_{T-\phi}>-\left(\Omega^{2} e m\left(x+e_{1}\right) \sin \theta\right)\left\{N_{B-v}^{\prime}\right\}<N_{T-\phi}> \\
& -\left(\omega^{2} m\right)\left\{N_{B-\nu}\right\}<N_{B-\nu}>+\left(\omega^{2} e m \sin \theta\right)\left\{N_{B-\nu}\right\}<N_{T-\phi}> \\
& \left.+(T)\left\{N_{B-v}^{\prime}\right\}<N_{B-v}^{\prime}>-\left(\Omega^{2} m\right)\left\{N_{B-v}\right\}<N_{B-v}>\right]\{u\}^{e}|J| d \xi
\end{aligned}
$$




$$
\begin{aligned}
& W_{t, \phi}^{e}=\int_{-1}^{+1}<\delta u>^{e}\left[(G J)\left\{N_{T-\phi}^{\prime}\right\}<N_{T-\phi}^{\prime}>-\left(\omega^{2} m \kappa_{m}^{2}\right)\left\{N_{T-\phi}\right\}<N_{T-\phi}>\right. \\
&-\left(\Omega^{2} e m\left(x+e_{1}\right) \sin \theta\right)\left\{N_{T-\phi}\right\}<N_{B-\nu}^{\prime}> \\
&+\left(\Omega^{2} e m\left(x+e_{1}\right) \cos \theta\right)\left\{N_{T-\phi}\right\}<N_{B-w}^{\prime}> \\
&+\left(\Omega^{2} e m \sin \theta\right)\left\{N_{T-\phi}\right\}<N_{B-\nu}> \\
&+\left(\omega^{2} e m \sin \theta\right)\left\{N_{T-\phi}\right\}<N_{B-\nu}> \\
&+\left(\Omega^{2} m\left(\kappa_{m 2}^{2}-\kappa_{m 1}^{2}\right) \cos 2 \theta\right)\left\{N_{T-\phi}\right\}<N_{T-\phi}> \\
&\left.-\left(\omega^{2} e m \cos \theta\right)\left\{N_{T-\phi}\right\}<N_{B-w}>\right]\{u\}^{e}|J| d \xi
\end{aligned}
$$

In order to obtain the unified governing equation of the element, equations (4.29) to (4.31) are added together to form the total element virtual work representing the discretized form of principle of virtual work (4.5):

$$
W_{\mathrm{int}}^{e}=W_{f, w}^{e}+W_{f, v}^{e}+W_{t, \phi}^{e}
$$

Leading to the following matrix form:

$$
W_{\mathrm{int}}^{e}=<\delta u>^{e}\left(-\omega^{2}[M]^{e}+[K]^{e}\right)\{u\}^{e}
$$

where $[M]^{e}$ and $[K]^{e}$ are the element mass and stiffness matrices, respectively, and are calculated from following equations:

$$
\begin{gathered}
{[M]^{e}=\int_{-1}^{+1}\left[(m)\left\{N_{B-w}\right\}<N_{B-w}>+(e m \cos \theta)\left\{N_{B-w}\right\}<N_{T-\phi}>\right.} \\
+(m)\left\{N_{B-v}\right\}<N_{B-v}>-(e m \sin \theta)\left\{N_{B-v}\right\}<N_{T-\phi}> \\
+\left(m \kappa_{m}^{2}\right)\left\{N_{T-\phi}\right\}<N_{T-\phi}>-(e m \sin \theta)\left\{N_{T-\phi}\right\}<N_{B-v}> \\
\left.+(e m \cos \theta)\left\{N_{T-\phi}\right\}<N_{B-w}>\right]|J| d \xi \\
{[K]^{e}=\left[K_{1}\right]^{e}+(T)^{e}\left[K_{2}\right]^{e}+\left(x+e_{1}\right)^{e}\left[K_{3}\right]^{e}}
\end{gathered}
$$




$$
\begin{aligned}
& {\left[K_{1}\right]^{e}=} \int_{-1}^{+1}\left[\left(E I_{y}\right)\left\{N_{B-w}^{n}\right\}<N_{B-w}^{n}>+\left(E I_{z y}\right)\left\{N_{B-w}^{n}\right\}<N_{B-v}^{n}>\right. \\
&+\left(E I_{z}\right)\left\{N_{B-v}^{n}\right\}<N_{B-v}^{n}>+\left(E I_{z y}\right)\left\{N_{B-v}^{n}\right\}<N_{B-w}^{n}> \\
&+(G J)\left\{N_{T-\phi}^{\prime}\right\}<N_{T-\phi}^{\prime}>+\left(\Omega^{2} e m \sin \theta\right)\left\{N_{B-v}\right\}<N_{T-\phi}> \\
&+\left(\Omega^{2} e m \sin \theta\right)\left\{N_{T-\phi}\right\}<N_{B-v}>-\left(\Omega^{2} m\right)\left\{N_{B-v}\right\}<N_{B-v}> \\
&\left.+\left(\Omega^{2} m\left(\kappa_{m 2}^{2}-\kappa_{m 1}^{2}\right) \cos 2 \theta\right)\left\{N_{T-\phi}\right\}<N_{T-\phi}>\right]|J| d \xi \\
& {\left[K_{3}\right]^{e}=\int_{-1}^{+1}\left[\left(\Omega^{2} e m \cos \theta\right)\left\{N_{B-w}^{\prime}\right\}<N_{T-\phi}>-\left(\Omega^{2} e m \sin \theta\right)\left\{N_{B-v}^{\prime}\right\}<N_{T-\phi}>\right.} \\
&+\left.\left(\Omega^{2} e m \sin \theta\right)\left\{N_{T-\phi}\right\}<N_{B-v}^{\prime}>-\left(\Omega^{2} e m \cos \theta\right)\left\{N_{T-\phi}\right\}<N_{B-w}^{\prime}>\right]|J| d \xi
\end{aligned}
$$

The system eigenproblem is then obtained by assembling the elements equations (using the well-known FEM assembly algorithm and connectivity table [32,33]):

$$
W_{\mathrm{int}}=\sum_{1}^{N E} W_{\mathrm{int}}^{e}=0
$$

or

$$
W_{\text {int }}=<\delta u>\left([K]-\omega^{2}[M]\right)\{u\}=0
$$

where

$$
[K]=\sum_{1}^{N E}[K]^{e}
$$

and

$$
[M]=\sum_{1}^{N E}[M]^{e}
$$

Since the virtual displacement $\langle\boldsymbol{\delta} \boldsymbol{u}>$ is arbitrary, then one can write: 


$$
\left([K]-\omega^{2}[M]\right)\{u\}=0
$$

The equation (4.43) is a linear eigenvalue problem in which the natural frequencies are eigenvalues and the vectors of nodal variables, mode shapes, are the eigenvectors. Equation (4.43) can be solved using any numerical method, and in this thesis MATLAB ${ }^{\circledR}$ and MAPLE $^{\odot}$ programs have been used to calculate the natural frequencies and mode shapes.

\subsection{Numerical Results}

To verify the formulation introduced in this chapter, the free vibrations of a certain number of illustrative beam configurations, exhibiting different couplings, were investigated and their natural frequencies and mode shapes were evaluated.

\subsubsection{Bending Vibrations of Uncoupled Beam}

As the first example, the out-of-plane flexural vibration of a beam was studied. The analytical solution for this beam is available (see Appendix A) and the natural frequencies and mode shapes can be found in Vibrations text books [34]. The FEM results can then be compared with the exact analytical solution, and the convergence of the FEM method can therefore be evaluated.

The beam parameters for the following example are considered as:

$$
E I_{\boldsymbol{y}}=1 ; \boldsymbol{m}=1 ; \boldsymbol{L}=1
$$

and the pre-twist angle of beam is zero. The differential equation of motion (2.1) for single uncoupled out-of-plane vibrations of beam reduces to: 


$$
\left(E I_{y} w^{n}\right)^{n}-\omega^{2} m w=0
$$

By implementing the Galerkin-type weighted residual formulation, the element discretized form of equation (4.44) can be written as:

$$
W_{f, w}^{e}=\int_{-1}^{+1}\left\{\delta w^{\prime \prime} E I_{y} w^{n}-\delta w \omega^{2} m w\right\} J d \xi
$$

The cubic Hermite shape functions were used and the FEM results along with the exact values obtained by analytical solution (see Appendix A) for the first five natural frequencies are shown in table 4.1.

Table 4.1: The first five natural frequencies of out-of-plane vibration of a cantilever beam

\begin{tabular}{cccccc}
\hline $\begin{array}{c}\text { Natural } \\
\text { Frequency } \\
(\text { rad/s) }\end{array}$ & $\begin{array}{c}\text { Exact } \\
\text { analytical } \\
\text { method [34] }\end{array}$ & $\begin{array}{c}\text { FEM } \\
\text { With 5 elements }\end{array}$ & $\begin{array}{c}\text { Error } \\
(\%) \\
\text { for } n= \\
5\end{array}$ & With 20 elements & $\begin{array}{c}\text { FEM } \\
\text { for } n=20\end{array}$ \\
\hline$\omega_{1}$ & 3.51602 & 3.51601 & 0.000 & 3.51603 & 0.000 \\
$\omega_{2}$ & 22.0345 & 22.0455 & 0.050 & 22.0345 & 0.000 \\
$\omega_{3}$ & 61.6972 & 61.9188 & 0.359 & 61.6982 & 0.002 \\
$\omega_{4}$ & 120.9019 & 122.3196 & 1.173 & 120.9094 & 0.006 \\
$\omega_{5}$ & 199.8595 & 203.0202 & 1.574 & 199.8933 & 0.010 \\
\hline
\end{tabular}

The mode shapes corresponding to the first three natural frequencies were calculated by the FEM method and are shown in Figure 4.2. 


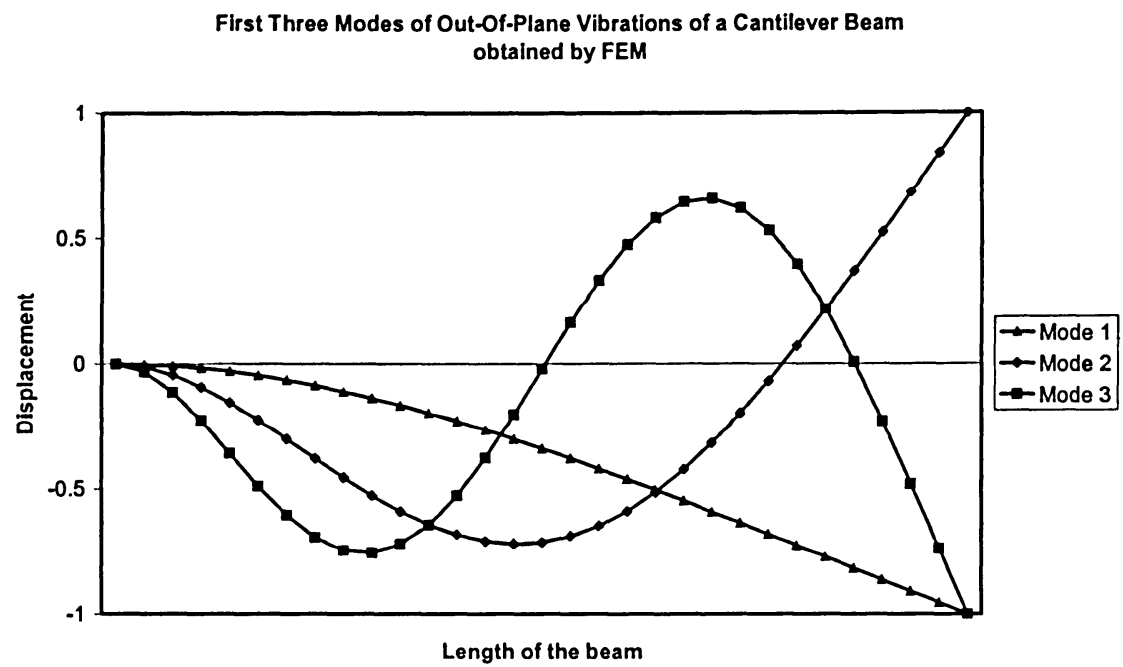

Figure 4.3: The first three mode of out-of-plane vibrations

of a cantilever beam based on FEM method.

\subsubsection{Coupled Bending-Torsion Vibrations}

For the case of coupled bending-torsion, the governing differential equations of motion (2.18) and (2.19) and the shear force and moments, equations (2.20) to (2.22), are coupled due to the rotating speed $\mathbf{\Omega}$ and eccentricity $\boldsymbol{e}$ effects. The mass and stiffness matrices, in this case, both contain coupling terms. Following a procedure similar to the pure bending case, the FEM final element equation changes to:

$$
W_{\mathrm{int}}^{e}=<\delta u>^{e}\left([K]^{e}-\omega^{2}[M]^{e}\right)\{u\}^{e}
$$

where:

$$
\begin{aligned}
{[M]^{e}=} & \int_{-1}^{+1}\left[(m)\left\{N_{B-w}\right\}<N_{B-w}>+(e m \cos \theta)\left\{N_{B-w}\right\}<N_{T-\phi}>\right. \\
& \left.+\left(m \kappa_{m}^{2}\right)\left\{N_{T-\phi}\right\}<N_{T-\phi}>+(e m \cos \theta)\left\{N_{T-\phi}\right\}<N_{B-w}>\right]|J| d \xi
\end{aligned}
$$


and

$$
[K]^{e}=\left[K_{1}\right]^{e}+(T)^{e}\left[K_{2}\right]^{e}+\left(x+e_{1}\right)^{e}\left[K_{3}\right]^{e}
$$

with

$$
\begin{gathered}
{\left[K_{1}\right]^{e}=\int_{-1}^{+1}\left[\left(E I_{y}\right)\left\{N_{B-w}^{\prime \prime}\right\}<N_{B-w}^{\prime \prime}>+(G J)\left\{N_{T-\phi}^{\prime}\right\}<N_{T-\phi}^{\prime}>\right.} \\
\left.+\left(\Omega^{2} m\left(\kappa_{m 2}^{2}-\kappa_{m 1}^{2}\right) \cos 2 \theta\right)\left\{N_{T-\phi}\right\}<N_{T-\phi}>\right]|J| d \xi \\
{\left[K_{2}\right]^{e}=\int_{-1}^{+1}\left[\left\{N_{B-w}^{\prime}\right\}<N_{B-w}^{\prime}>\right]|J| d \xi} \\
{\left[K_{3}\right]^{e}=\int_{-1}^{+1}\left[\left(\Omega^{2} e m \cos \theta\right)\left\{N_{B-w}^{\prime}\right\}<N_{T-\phi}>-\left(\Omega^{2} e m \cos \theta\right)\left\{N_{T-\phi}\right\}<N_{B-w}^{\prime}>\right]|J| d \xi}
\end{gathered}
$$

\subsubsection{Non-rotating Beam}

Aircraft wings and thin-walled open section beams fall in this category, since, in these cases, the equations governing bending and torsion displacements are coupled due to the eccentricity between the mass and elastic axes.

As the first example, the natural frequencies of a typical cantilever uniform aircraft wing were calculated. The beam specifications are:

$$
\begin{aligned}
& \boldsymbol{L}=6.0 \mathrm{~m} \\
& \boldsymbol{e}=0.18 \mathrm{~m} \\
& \boldsymbol{m}=35.75 \mathrm{~kg} / \mathrm{m} \\
& \boldsymbol{E I}=9.75 \mathrm{MN} \cdot \mathrm{m}^{2} \\
& \boldsymbol{G J}=0.988 \mathrm{MN} \cdot \mathrm{m}^{2} \\
& \boldsymbol{m} . \boldsymbol{k}_{\boldsymbol{m}}{ }^{2}=8.65 \mathrm{~kg} . \mathrm{m}^{2}
\end{aligned}
$$


and the FEM results were compared with the exact solution obtained from the DSM method [22] (Table 4.2):

Table 4.2: The first six natural frequencies of coupled bending-torsion vibrations of a cantilever beam

\begin{tabular}{cccc}
\hline $\begin{array}{c}\text { Natural Frequency } \\
(\mathrm{rad} / \mathrm{s})\end{array}$ & $\begin{array}{c}\text { Exact } \\
\text { Method (DSM) }\end{array}$ & $\begin{array}{c}\text { FEM } \\
n=30\end{array}$ & $\begin{array}{c}\text { Error of FEM } \\
(\%)\end{array}$ \\
\hline$\omega_{1}$ & 49.62 & 49.62 & 0.01 \\
$\omega_{2}$ & 97.04 & 97.05 & 0.01 \\
$\omega_{3}$ & 248.87 & 249.07 & 0.08 \\
$\omega_{4}$ & 355.59 & 355.78 & 0.05 \\
$\omega_{5}$ & 451.46 & 452.58 & 0.25 \\
$\omega_{6}$ & 610.32 & 613.59 & 0.54 \\
\hline
\end{tabular}

As the second example, an aircraft wing, modeled by three uniform beams with different cross section parameters, was studied to show the flexibility and applicability of the FEM method. The beam is shown in Figure 4.3, and the beam parameters are given in Table 4.3.

Table 4.3: The mechanical and geometrical parameters of the three-beam wing

\begin{tabular}{|c|c|c|c|}
\hline & Section I & Section II & Section III \\
\hline$L=6.0 \mathrm{~m}$ & $l_{1}=2 \mathrm{~m}$ & $l_{2}=2 m$ & $l_{3}=2 \mathrm{~m}$ \\
\hline$m=35.75 \mathrm{~kg} / \mathrm{m}$ & $m_{1}=m$ & $m_{2}=(2 / 3) * m$ & $m_{3}=(1 / 3) * m$ \\
\hline$m . k_{m}^{2}=8.65 \mathrm{~kg} . \mathrm{m}$ & $\left(m \cdot k_{m}^{2}\right)_{l}=m \cdot k_{m}^{2}$ & $\left(m \cdot k_{m}^{2}\right)_{2}=(2 / 3) m \cdot k_{m}^{2}$ & $\left(m \cdot k_{m}{ }^{2}\right)_{3}=(1 / 3) m \cdot k_{m}{ }^{2}$ \\
\hline$E I=9.75 M N \cdot m^{2}$ & $E I_{1}=E I$ & $E I_{2}=(2 / 3) * E I$ & $E I_{3}=(1 / 3) \star E I$ \\
\hline $\boldsymbol{G J}=0.988 M N \cdot \mathrm{m}^{2}$ & $G J_{1}=G J$ & $G J_{2}=(2 / 3) * G J$ & $G J_{3}=(1 / 3) * G J$ \\
\hline$e=0.18 \mathrm{~m}$ & $e=0.18 m$ & $e=0.18 \mathrm{~m}$ & $e=0.18 \mathrm{~m}$ \\
\hline
\end{tabular}




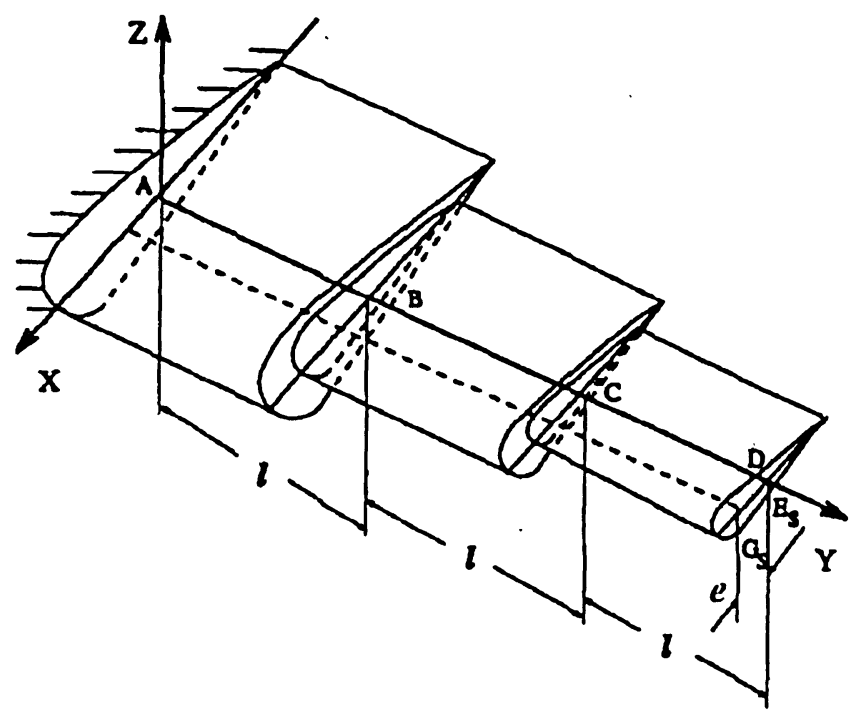

Figure 4.4: The three-beam airplane wing with coordinate system and geometry.

The coupled natural frequencies obtained from the FEM and the exact DSM method results [22] are presented in Table 4.4. The convergence test results are also shown in Figure 4.4.

Table 4.4: The first five natural frequencies of the three-beam aircraft wing

\begin{tabular}{cccc}
\hline $\begin{array}{c}\text { Natural Frequency } \\
(\mathrm{rad} / \mathrm{s})\end{array}$ & $\begin{array}{c}\text { Exact Method } \\
(\mathrm{DSM})\end{array}$ & $\begin{array}{c}\text { FEM } \\
n=15\end{array}$ & $\begin{array}{c}\text { Error of FEM } \\
(\%)\end{array}$ \\
\hline$\omega_{1}$ & 74.43 & 74.43 & 0.00 \\
$\omega_{2}$ & 128.57 & 128.58 & 0.00 \\
$\omega_{3}$ & 253.40 & 253.56 & 0.06 \\
$\omega_{4}$ & 376.59 & 376.96 & 0.10 \\
$\omega_{5}$ & 431.29 & 431.97 & 0.16 \\
\hline
\end{tabular}




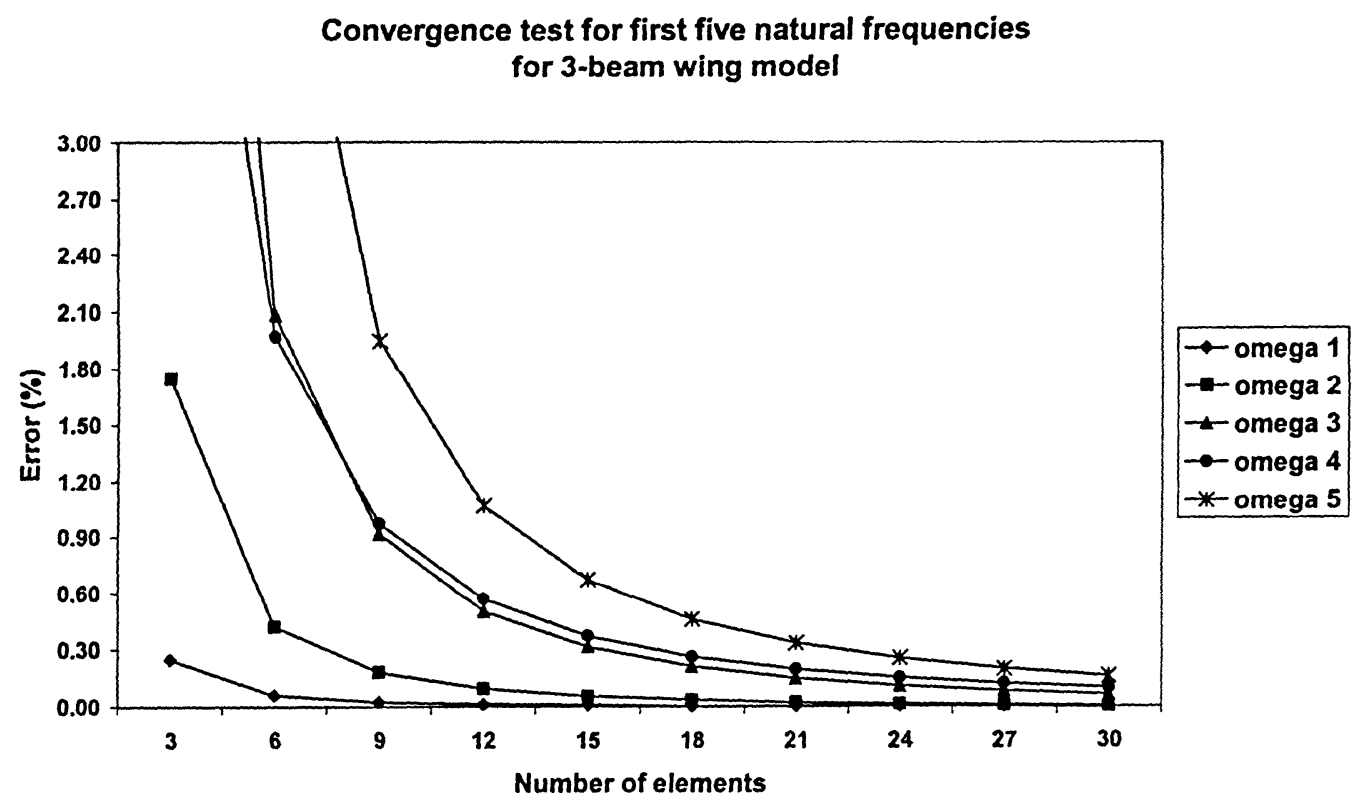

Figure 4.5: The FEM convergence results for three-beam wing.

As it can be seen, the FEM method exhibits satisfactory convergence rates, where the error for the first natural frequency obtained by a six-element model is $0.06 \%$, and for the first five frequencies a model made of 30 elements ends up with errors less than $0.2 \%$.

\subsubsection{Axially Loaded Beam}

The analysis of an axially loaded beam can be considered as the first step in the modeling of rotating systems such as helicopter, propeller and turbine blades, where the element centrifugal force is replaced by a constant axial load. The differential equations of motion governing coupled bending-torsion vibrations of an axially loaded beam have been introduced in [23]:

$$
\begin{gathered}
E I_{y} w^{\prime \prime \prime}-\left(T w^{\prime}\right)^{\prime}+T e \phi^{n}-\omega^{2} m(w-e \phi)=0 \\
-\left(G J+T \kappa_{m}^{2}\right) \phi^{n}+T e w^{n}-\omega^{2} m\left(\kappa_{m}^{2} \phi-e w\right)=0
\end{gathered}
$$


where the force boundary conditions at free end, in this case, are:

$$
\begin{gathered}
V_{z}=-E I_{y} w^{\prime \prime}+T\left(w^{\prime}-e \phi^{\prime}\right)=0 \\
M_{x}=\left(G J+T \kappa_{m}{ }^{2}\right) \phi^{\prime}-T e w^{\prime}=0 \\
M_{y}=E I_{y} w^{\prime \prime}=0
\end{gathered}
$$

In order to investigate the vibrational behaviour of axially loaded beams, a cantilever beam with semi-circular cross section (Figure 4.5) and the following properties was studied $[20,23]$ :

$$
\begin{aligned}
& \boldsymbol{L}=0.82 \mathrm{~m} \\
& \boldsymbol{e}=0.0155 \mathrm{~m} \\
& \boldsymbol{m}=0.835 \mathrm{~kg} / \mathrm{m} \\
& \boldsymbol{E} \boldsymbol{I}=6380.14 \mathrm{~N} \cdot \mathrm{m}^{2} \\
& \boldsymbol{G} \boldsymbol{J}=43.46 \mathrm{~N} . \mathrm{m}^{2} \\
& \boldsymbol{m} \cdot \boldsymbol{k}_{\boldsymbol{m}}{ }^{2}=0.000501 \mathrm{~kg} \cdot \mathrm{m}
\end{aligned}
$$




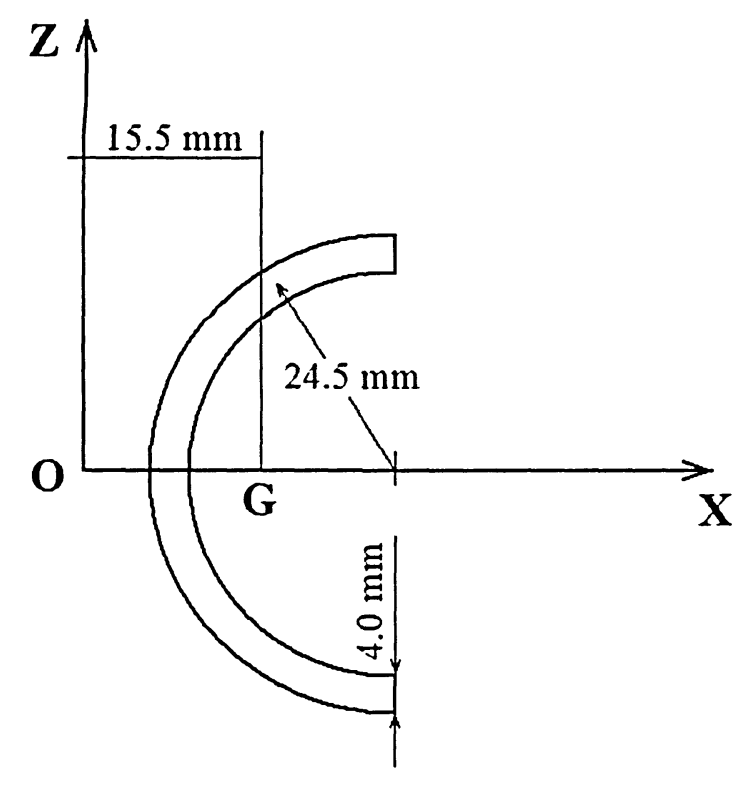

Figure 4.6: The bending-torsion coupled beam cross-sectional geometry.

The free vibrations of beam in two different situations were studied. First, the axial force was considered to be zero, $\boldsymbol{T}=\mathbf{0}$, and the first five natural frequencies of the beam were evaluated (Table 4.5):

Table 4.5: The bending-torsion natural frequencies of the cantilever beam (no axial load $(T=0)$ )

\begin{tabular}{cccc}
\hline $\begin{array}{c}\text { Natural Frequency } \\
(\mathrm{Hz})\end{array}$ & $\begin{array}{c}\text { Reference } \\
\text { Frequency } \\
{[20]}\end{array}$ & $\begin{array}{c}\text { FEM with } \\
20 \text { elements }\end{array}$ & $\begin{array}{c}\text { Error of FEM } \\
(\%)\end{array}$ \\
\hline$\omega_{1}$ & 62.60 & 62.61 & 0.02 \\
$\omega_{2}$ & 130.18 & 130.21 & 0.02 \\
$\omega_{3}$ & 261.15 & 261.66 & 0.20 \\
$\omega_{4}$ & 421.36 & 423.59 & 0.53 \\
$\omega_{5}$ & 612.09 & 618.52 & 1.05 \\
\hline
\end{tabular}


If the axial force is applied to the beam, then the natural frequencies will change. Including a compressive force $T=-1790(N)$, the natural frequencies have been sought and shown in Table 4.6.

Table 4.6: The bending-torsion natural frequencies of the axially loaded cantilever beam $(T=-1790 N)$

\begin{tabular}{cccc}
\hline $\begin{array}{c}\text { Natural Frequency } \\
(\mathrm{Hz})\end{array}$ & $\begin{array}{c}\text { Reference } \\
\text { Frequency } \\
{[20]}\end{array}$ & $\begin{array}{c}\text { FEM with } \\
20 \text { elements }\end{array}$ & $\begin{array}{c}\text { Error of FEM } \\
(\%)\end{array}$ \\
\hline$\omega_{1}$ & 60.23 & 60.24 & 0.02 \\
$\omega_{2}$ & 128.42 & 128.45 & 0.02 \\
$\omega_{3}$ & 257.96 & 258.46 & 0.19 \\
$\omega_{4}$ & 415.54 & 417.74 & 0.53 \\
$\omega_{5}$ & 604.60 & 611.12 & 1.08 \\
\hline
\end{tabular}

One can see that the natural frequencies for a beam with compressive axial force are smaller than those of same beam without axial load. This can be explained by the fact that the compressive axial force decreases the flexural rigidity of the beam, while tensile axial force increases the so-called geometric stiffness of the system [23].

\subsubsection{Non-rotating Pre-twisted Beam}

For pre-twisted beam the coupling between flap-wise bending and torsion displacements, equations (2.18) and (2.19), occurs because of eccentricity, $e$, and pretwist angle, $\theta$, appear in coupling terms. As an example, the FEM analysis of a beam with following characteristics was studied:

$$
\begin{aligned}
& \boldsymbol{L}=40.0 \text { in } \\
& \boldsymbol{e}=0.4 \text { in } \\
& \boldsymbol{\theta}=0 \text { degree }
\end{aligned}
$$




$$
\begin{aligned}
& \boldsymbol{m}=0.0015 \mathrm{slug} / \mathrm{in} \\
& \boldsymbol{k}_{\boldsymbol{m} \mathbf{I}}{ }^{2}=0.18 \mathrm{in}^{2} \\
& \boldsymbol{k}_{\boldsymbol{m} 2}{ }^{2}=0.71 \mathrm{in}^{2} \\
& E I=25000 \mathrm{lb} \cdot \mathrm{in}^{2} \\
& G J=9000 \mathrm{lb} \cdot \mathrm{in}^{2}
\end{aligned}
$$

The FEM results for the first five natural frequencies were calculated and compared to the reference values obtained from the Integrating Matrix Method (IMM) [2] (see Table 4.7).

Table 4.7: The bending-torsion natural frequencies of the pre-twisted clamped-free beam

\begin{tabular}{cccc}
\hline $\begin{array}{c}\text { Natural } \\
\text { Frequency } \\
\text { (rad/s) }\end{array}$ & $\begin{array}{c}\text { IMM } \\
\text { Method } \\
\text { (15 stations) }\end{array}$ & $\begin{array}{c}\text { FEM element } \\
\text { With } \\
30 \text { elements }\end{array}$ & $\begin{array}{c}\text { Error of FEM } \\
\text { method } \\
(\%)\end{array}$ \\
\hline$\omega_{1}$ & 31.05 & 31.059 & 0.029 \\
$\omega_{2}$ & 193.74 & 193.748 & 0.004 \\
$\omega_{3}$ & 390.87 & 390.923 & 0.014 \\
$\omega_{4}$ & 539.54 & 539.598 & 0.011 \\
$\omega_{5}$ & 1043.94 & 1044.306 & 0.035 \\
\hline
\end{tabular}

All examples presented in this section confirmed the applicability and good convergence of the FEM formulation developed for coupled bending-torsion beam vibrations.

\subsubsection{Coupled Bending-Bending Vibrations}

For the coupled flap-lag vibrations, the equations of motion (2.1) and (2.2) reduce to (2.12) and (2.13), where the natural (force) boundary conditions at free end can be written as equations (2.14) to (2.17). 
As already stated, asymmetric geometry leads to the coupling terms in the equations. The rotating speed, $\Omega$, appears in both centrifugal force, $T$, and centripetal term, $-\mathbf{\Omega}^{2} \boldsymbol{m} \boldsymbol{v}$. The centrifugal force increases the system stiffness and the centripetal force, due to its negative sign, decreases the rigidity of the beam for in-plane vibrations $[16,21]$. The resulting element mass and stiffness matrices produced by FEM approach can then be written as:

$$
[M]^{e}=\int_{-1}^{+1}\left[(m)\left\{N_{B-w}\right\}<N_{B-w}>+(m)\left\{N_{B-v}\right\}<N_{B-v}>\right]|J| d \xi
$$

and

$$
[K]^{e}=\left[K_{1}\right]^{e}+(T)^{e}\left[K_{2}\right]^{e}
$$

where

$$
\begin{aligned}
{\left[K_{1}\right]^{e}=} & \int_{-1}^{+1}\left[\left(E I_{y}\right)\left\{N_{B-w}^{\prime \prime}\right\}<N_{B-w}^{\prime \prime}>+\left(E I_{z y}\right)\left\{N_{B-w}^{\prime \prime}\right\}<N_{B-v}^{\prime \prime}>\right. \\
& +\left(E I_{z}\right)\left\{N_{B-v}^{\prime \prime}\right\}<N_{B-v}^{\prime \prime}>+\left(E I_{z y}\right)\left\{N_{B-v}^{\prime \prime}\right\}<N_{B-w}^{\prime \prime}> \\
& \left.-\left(\Omega^{2} m\right)\left\{N_{B-v}\right\}<N_{B-v}>\right]|J| d \xi
\end{aligned}
$$

and

$$
\left[K_{2}\right]^{e}=\int_{-1}^{+1}\left[\left\{N_{B-w}^{\prime}\right\}<N_{B-w}^{\prime}>+\left\{N_{B-v}^{\prime}\right\}<N_{B-\nu}^{\prime}>\right]|J| d \xi
$$

The rotating beam analyzed in this section is a scaled model of propeller blade WADC S-5 [2,16,27], which is 24 " long with a hub radius (offset) of 6 ". The mechanical and geometrical properties of the blade are given in table 4.8 . 
Table 4.8: The properties of the scaled propeller blade model WADC S-5, with hub radius $e_{1}=6$ (in) and blade length $L=18$ (in)

\begin{tabular}{|c|c|c|c|c|c|}
\hline $\begin{array}{l}\text { Section } \\
\text { number }\end{array}$ & $\begin{array}{l}x, \\
\text { in }\end{array}$ & $\underset{l b . s^{2} / i n^{2}}{m}$ & $\underset{\mid b . i n^{2}}{\left.E\right|_{\xi,}}$ & $\begin{array}{c}E I_{n,} \\
I b . i n^{2}\end{array}$ & $\begin{array}{c}\theta, \\
d e g\end{array}$ \\
\hline 1 & 0.0 & $1.026 \times 10^{-3}$ & $0.200 \times 10^{6}$ & $63 \times 10^{6}$ & 30.5 \\
\hline 2 & 2.0 & 0.696 & 0.110 & 49 & 25.2 \\
\hline 3 & 4.0 & 0.660 & 0.083 & 46 & 20.1 \\
\hline 4 & 6.0 & 0.608 & 0.058 & 44 & 14.8 \\
\hline 5 & 8.0 & 0.564 & 0.042 & 43 & 9.6 \\
\hline 6 & 10.0 & 0.535 & 0.031 & 43 & 4.7 \\
\hline 7 & 12.0 & 0.520 & 0.027 & 44 & 0.0 \\
\hline 8 & 14.0 & 0.506 & 0.026 & 47 & -4.2 \\
\hline 9 & 16.0 & 0.498 & 0.025 & 51 & -7.5 \\
\hline 10 & 18.0 & 0.498 & 0.024 & 56 & -10.0 \\
\hline
\end{tabular}

The flexural rigidities in global coordinates at each point along the length of the beam can be obtained by following equations [16] (see Figure 2.1).

$$
\begin{aligned}
& E I_{z}=E I_{\xi} \cos ^{2} \theta+E I_{\eta} \sin ^{2} \theta \\
& E I_{y}=E I_{\eta} \cos ^{2} \theta+E I_{\xi} \sin ^{2} \theta \\
& E I_{z y}=\left(E I_{\xi}-E I_{\eta}\right) \sin \theta \cos \theta
\end{aligned}
$$

where the $E I_{\xi}$ and $E I_{\eta}$ flexural rigidities are evaluated with respect to the element principal coordinates whereas $\boldsymbol{E} \boldsymbol{I}_{\boldsymbol{y}}$ and $\boldsymbol{E} \boldsymbol{I}_{\boldsymbol{z}}$ are those calculated with respect to the global coordinates; $\theta$ is the pre-twist angle assumed to be constant along each element. The natural frequencies obtained from FEM method are shown in Table 4.9. The first three natural frequencies for different angular velocities were obtained and compared with the experimental values $[2,5,16,27]$. It can be seen that comparing with other numerical approaches [2] and [5], the frequencies obtained from the FEM are close enough to the experimental results. This verifies the validity of FEM formulations and its good convergence for coupled bending-bending vibrations. The mode shapes for three different angular speeds of blade were investigated and shown in Figures 4.6 to 4.8. 
Table 4.9: The natural frequencies $(\mathrm{Hz})$ of the first three modes of vibrations of propeller blade WADC S-5

\begin{tabular}{|c|c|c|c|c|c|}
\hline $\begin{array}{l}\text { Blade rotary } \\
\text { speed (rpm) }\end{array}$ & $\begin{array}{c}\begin{array}{c}\text { Mode } \\
\text { numbe } \\
r \\
\end{array} \\
\end{array}$ & $\begin{array}{l}\text { FEM } \\
n=9\end{array}$ & $\begin{array}{c}\text { Result of } \\
\text { Reference } \\
\text { [2] }\end{array}$ & $\begin{array}{c}\text { Result of } \\
\text { Reference } \\
\text { [5] }\end{array}$ & $\begin{array}{c}\text { Experime } \\
n t \\
{[27]} \\
\end{array}$ \\
\hline \multirow{3}{*}{1567} & 1 & 40.32 & 40.96 & 39.89 & 40.08 \\
\hline & 2 & 108.49 & 109.22 & 107.40 & \\
\hline & 3 & 279.27 & 279.79 & 276.32 & \\
\hline \multirow{3}{*}{1589} & 1 & 40.70 & 41.35 & 40.26 & \\
\hline & 2 & 109.01 & 109.77 & 107.93 & 107.53 \\
\hline & 3 & 279.92 & 280.47 & 276.97 & \\
\hline \multirow{3}{*}{2609} & 1 & 58.99 & 60.07 & 58.05 & 58.73 \\
\hline & 2 & 137.18 & 139.52 & 135.99 & \\
\hline & 3 & 316.82 & 309.40 & 313.98 & \\
\hline \multirow{3}{*}{2614} & 1 & 59.08 & 60.16 & 58.14 & \\
\hline & 2 & 137.33 & 139.68 & 136.14 & 137.02 \\
\hline & 3 & 317.03 & 319.62 & 314.19 & \\
\hline \multirow{3}{*}{3583} & 1 & 76.87 & 78.34 & 75.30 & 76.52 \\
\hline & 2 & 168.08 & & 166.25 & 170.60 \\
\hline & 3 & 361.38 & & 357.70 & \\
\hline \multirow{3}{*}{4486} & 1 & 93.48 & & 91.42 & 93.07 \\
\hline & 2 & 197.91 & & & \\
\hline & 3 & 407.24 & & & \\
\hline \multirow{3}{*}{4537} & 1 & 94.42 & & & \\
\hline & 2 & 199.61 & & 196.41 & 202.53 \\
\hline & 3 & 409.91 & & & \\
\hline \multirow{3}{*}{5884} & 1 & 119.17 & & 116.07 & 117.50 \\
\hline & 2 & 244.05 & & & \\
\hline & 3 & 481.05 & & & \\
\hline
\end{tabular}



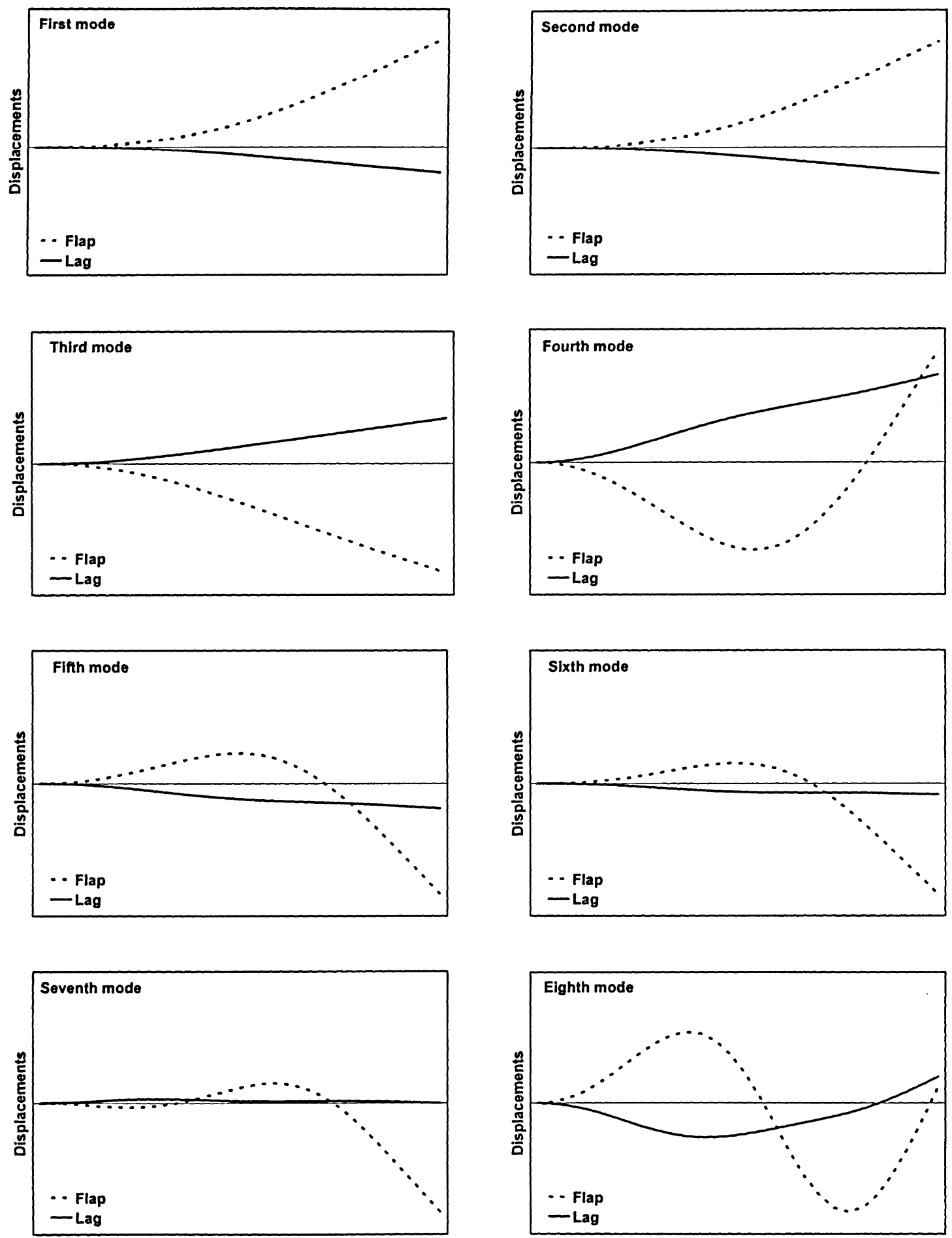

Figure 4.7: The first eight modes of the $W A D C S-5$ propeller blade for $\Omega=0$. 

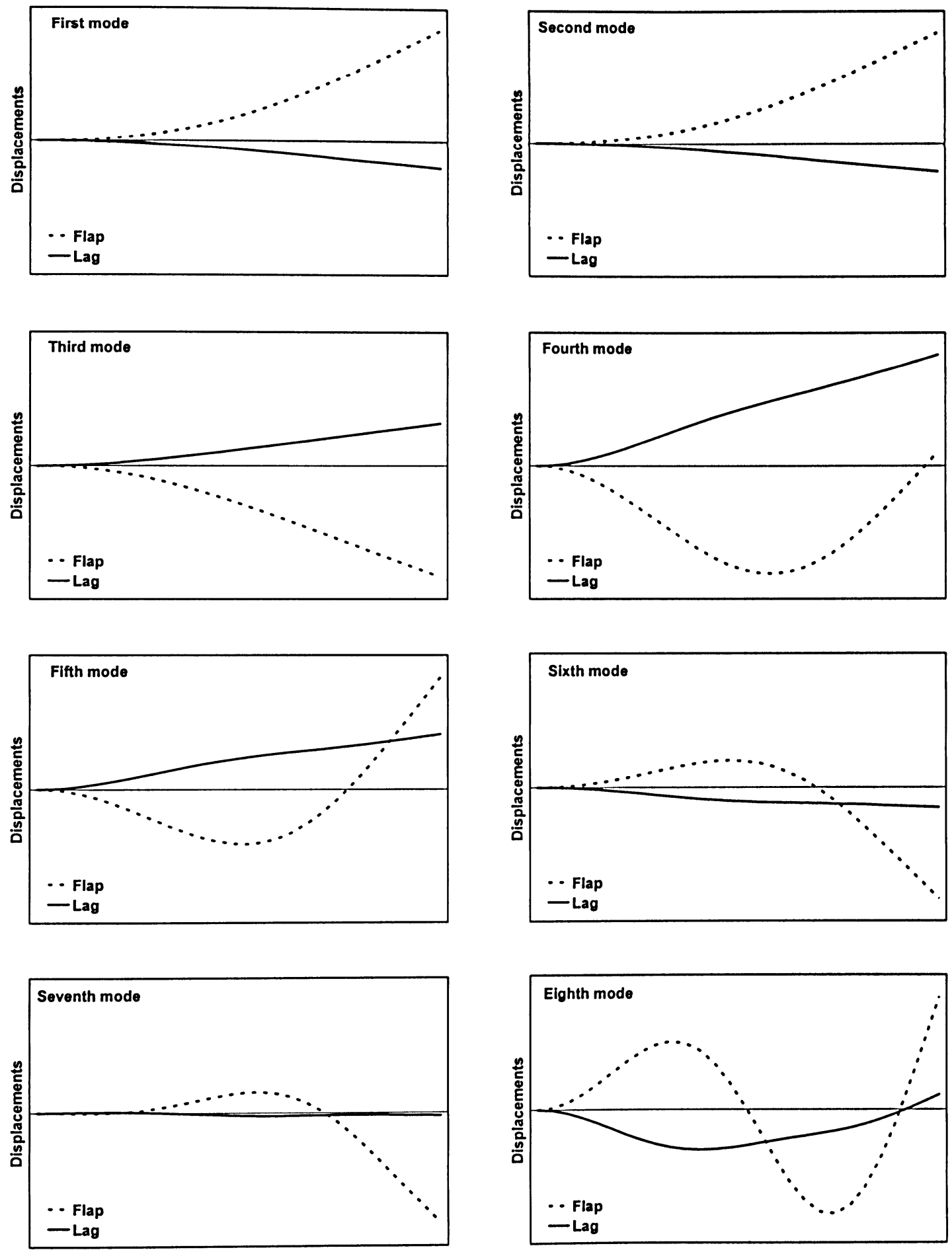

Figure 4.8: The first eight mode shapes of the $W A D C S-5$ propeller blade for $\Omega=1567 \mathrm{rpm}$. 

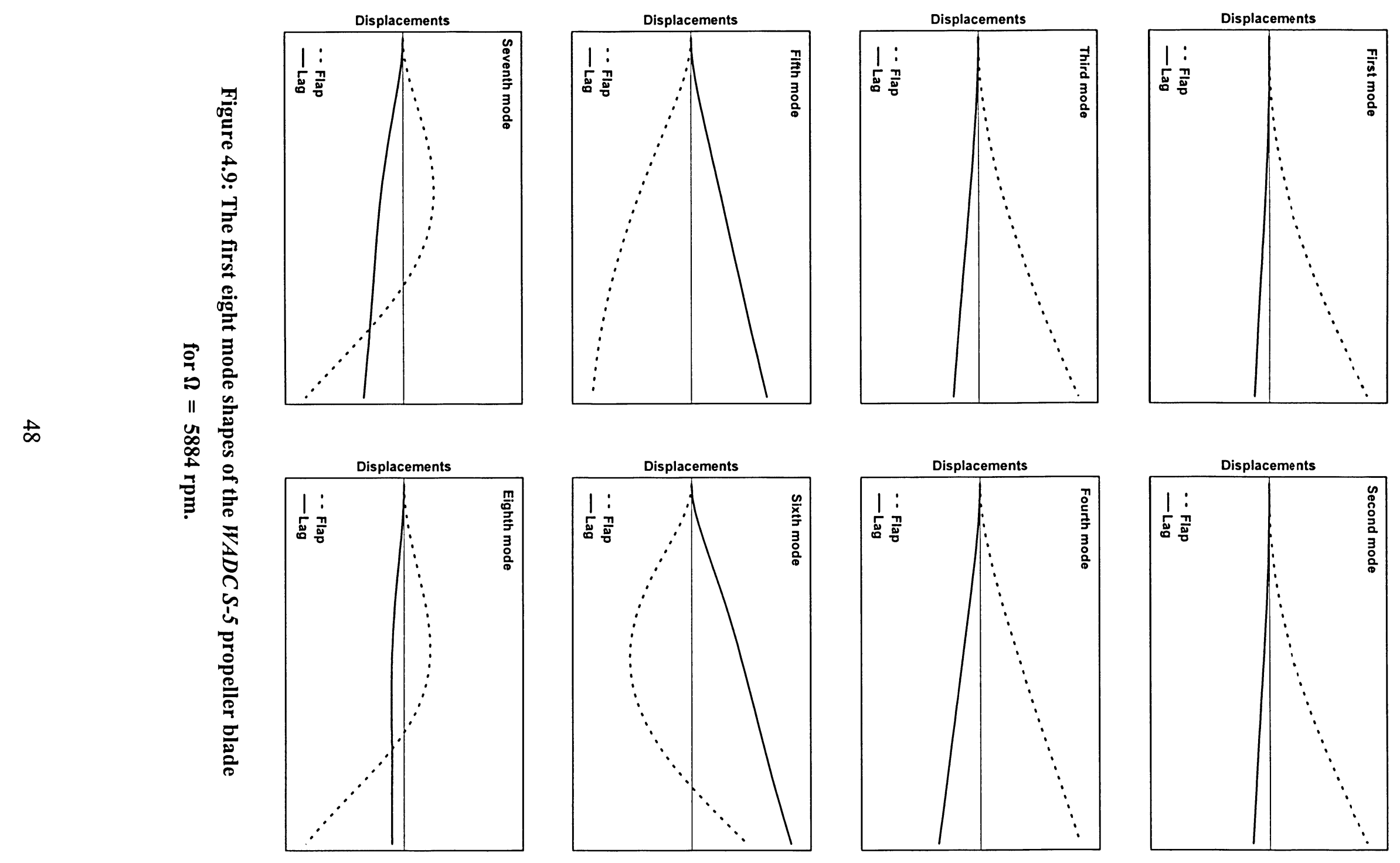
One can see that by increasing the angular velocity modes exhibit less deformations since the centrifugal force increases the blade stiffness. Comparing flap mode shapes for two cases, $\mathbf{\Omega}=1567$ (rpm) and $\mathbf{\Omega}=5884$ (rpm), shows that increasing the angular velocity of the blade leads to different mode shapes in higher frequencies (see for example the $8^{\text {th }}$ mode shapes).

\subsubsection{Coupled Bending-Bending-Torsion Vibrations}

In previous sections, the coupling between out-of-plane (flap) bending and torsion displacements and also the coupling between flap and in-plane (lag) displacements for rotating and non-rotating beam structures were studied. In this section, the free vibration analysis of the triply coupled bending-bending-torsion beams is investigated.

\subsubsection{Vibrations of Non-rotating Uncoupled Beam}

If none of the parameters $\Omega, e, \theta$ appears in the equations of motion, for a symmetric uniform beam, the equations are uncoupled and reduce to the well-known forms of pure bending vibration and pure torsion vibration of a beam (see Appendix A).

To find dimensionless natural frequencies, the beam parameters $E I_{y}, E I_{z}, G J, m$ and $\kappa_{m}$ are considered to be unit. The FEM results for such a beam are presented in table 4.10 .

Table 4.10: The uncoupled bending, bending and torsion natural frequencies of the clamped-free beam

\begin{tabular}{cccccccccc}
\hline $\begin{array}{c}\text { Natural Frequency } \\
\text { (rad/s) }\end{array}$ & $\omega_{1}$ & $\omega_{2,3}$ & $\omega_{4}$ & $\omega_{5}$ & $\omega_{6}$ & $\omega_{7}$ & $\omega_{8}$ & $\omega_{9}$ & $\omega_{10,11}$ \\
\hline $\begin{array}{c}\text { FEM element } \\
\text { With 20 elements }\end{array}$ & 1.571 & 3.516 & 4.723 & 7.905 & 11.135 & 14.433 & 17.820 & 21.315 & 22.035 \\
\hline
\end{tabular}


It can be seen that the natural frequencies evaluated from uncoupled bendingbending-torsion equations are equal to the one calculated by separate equations. It means that $\omega_{2,3}$ and $\omega_{10,11}$ are repeated natural frequencies corresponding to the first and second frequencies of the pure lateral vibrations of a cantilever beam, and $\omega_{1}$ and $\omega_{4}$ are the first two frequencies corresponding to the torsional vibrations of the beam.

It is also to be mentioned that since a mesh of only 20 elements has been used, then the third and higher frequencies of torsion vibrations are not accurate enough. Investigation in error shows that for $\omega_{5}=7.905$ the error is $0.65 \%$, for $\omega_{6}=11.135$ the error is $1.27 \%$ and the error increases up to repeated frequencies $\omega_{10,11}=22.035$, for which the error decreases to $0.005 \%$. This means the first two frequencies of each uncoupled vibrations are obtained accurately by only 20 elements.

\subsubsection{Vibrations of Non-rotating Coupled Beam}

A good aircraft wing-beam model exhibits the triply coupled flap-lag-torsion Vibrational behaviour. To verify the validity of the presented FEM method for triply coupled vibrations, a beam with the following properties was investigated $[2,16,28,29]$ :

$$
\begin{aligned}
& L=40.0 \mathrm{in} \\
& e=1.414 \mathrm{in} \\
& \theta=45 \mathrm{deg} \\
& \boldsymbol{m}=0.0015 \mathrm{slug} / \mathrm{in} \\
& \boldsymbol{k}_{\boldsymbol{m} \boldsymbol{I}}=\boldsymbol{k}_{\boldsymbol{m} 2}=1.0 \mathrm{in} \\
& E I_{\eta}=25000 \mathrm{lb} \cdot \mathrm{in}^{2} \\
& E I_{\xi}=75000 \mathrm{lb} \cdot \mathrm{in}^{2} \\
& G J=9000 \mathrm{lb} \cdot \mathrm{in}^{2}
\end{aligned}
$$


The flexural rigidities $E I_{z}, E I_{y}$ and $E I_{\mathfrak{z}}$ are evaluated from equations (4.56), (4.57) and (4.58). The FEM results for natural frequencies of the coupled beam are shown in table 4.11. The convergence rates of the FEM method for the first four frequencies were also investigated and are shown in Figure 4.9.

Table 4.11: The coupled bending-bending-torsion natural frequencies of the pre-twisted cantilever beam

\begin{tabular}{cccccc}
\hline $\begin{array}{c}\text { Natural Frequency } \\
(\mathrm{rad} / \mathrm{s})\end{array}$ & TMM [2] & $\begin{array}{c}\text { FEM } \\
n=5\end{array}$ & $\begin{array}{c}\text { Error (\%) } \\
\text { for } n=5\end{array}$ & $\begin{array}{c}\text { FEM } \\
n=10\end{array}$ & $\begin{array}{c}\text { Error (\%) } \\
\text { for } n=10\end{array}$ \\
\hline$\omega_{1}$ & 30.8295 & 30.8320 & 0.008 & 30.8300 & 0.002 \\
$\omega_{2}$ & 53.8277 & 53.8285 & 0.001 & 53.8278 & 0.000 \\
$\omega_{3}$ & 184.6175 & 185.1379 & 0.282 & 184.7376 & 0.065 \\
$\omega_{4}$ & 337.3333 & 337.5015 & 0.050 & 337.3440 & 0.003 \\
$\omega_{5}$ & 484.3373 & 493.1382 & 1.817 & 486.4762 & 0.442 \\
\hline
\end{tabular}

Convergence test of first four frequencies for non-rotating triply coupled beam

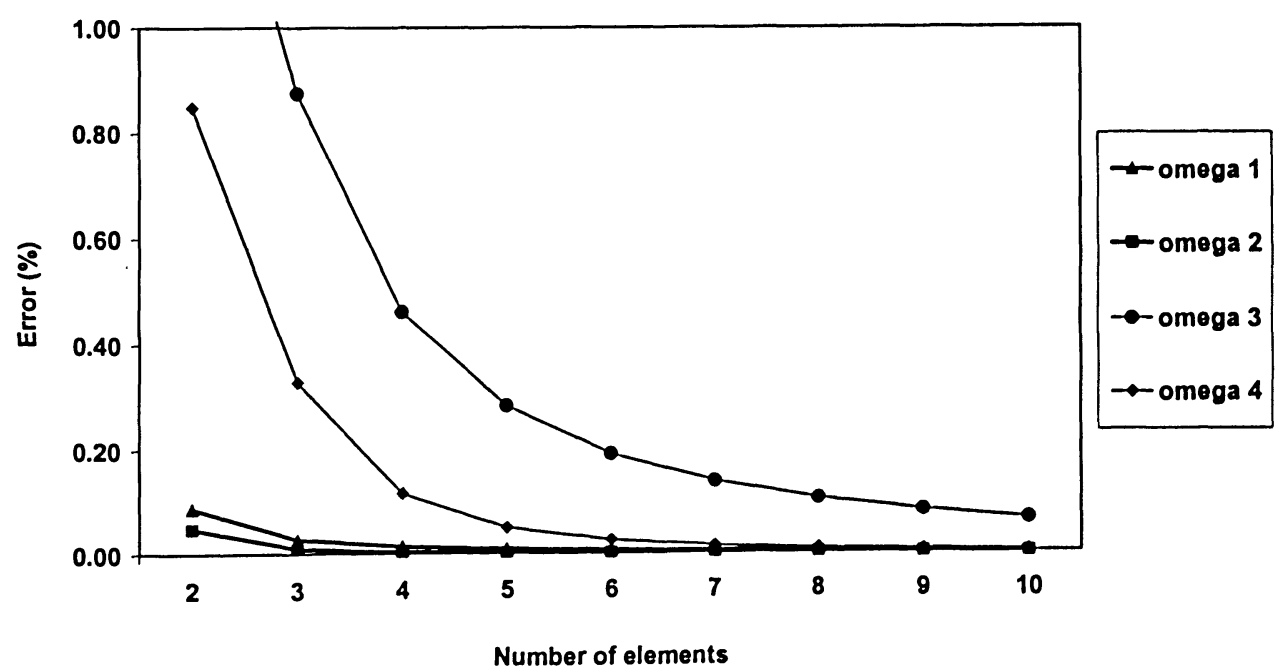

Figure 4.10: The FEM convergence results for non-rotating triply coupled beam. 
One can see the FEM convergence rates are quite satisfactory where for first three natural frequencies as a five-element model results in an error less than $0.1 \%$.

\subsubsection{Vibrations of Rotating Triply Coupled Beam}

Triply coupled rotating beam can be used in low frequency calculation of some helicopter blades, propeller blades and also compressor/turbine blades. The coupled bending-bending-torsion vibrations of a uniform rotating beam with a pre-twist angle are governed by equations (2.1), (2.2) and (2.3). In order to investigate the dynamic behaviour of such beams, a helicopter blade reported in literature [6] was investigated. The blade in this section is considered to be cantilever, while in the real model the blade is connected to rotating hub with some load paths [6]. The FEM results are shown in Tables 4.12 and 4.13 for non-rotating $(\boldsymbol{\Omega}=0)$ and rotating $(\boldsymbol{\Omega}=360 \mathrm{rpm})$, where the properties of beam are:

$$
\begin{aligned}
& L=208 \text { in } \\
& e=-0.6 \text { in } \\
& e_{1}=52 \text { in } \\
& \theta=15.026 \mathrm{deg} \\
& \boldsymbol{m}=0.0015 \mathrm{lb} \cdot \mathrm{sec}^{2} / \mathrm{in}^{2} \\
& \boldsymbol{m} . \boldsymbol{k}_{\boldsymbol{m} \boldsymbol{l}}{ }^{2}=0.89545 \times 10^{-3} \quad \mathrm{lb}_{\mathrm{sec}}{ }^{2} \\
& m . k_{m 2}{ }^{2}=0.04 \text { lb.sec }{ }^{2} \\
& E I_{\eta}=0.2977 \times 10^{8} l b . i n^{2} \\
& E I_{\xi}=10^{9} \quad l b . i n^{2} \\
& \boldsymbol{G J}=0.2 \times 10^{8} l b . \mathrm{in}^{2}
\end{aligned}
$$




\begin{tabular}{|c|c|c|c|}
\hline $\begin{array}{l}\text { Frequency } \\
\text { (rad/s) }\end{array}$ & $\begin{array}{l}\text { Triple load path } \\
\text { TMM method [6] }\end{array}$ & $\begin{array}{c}F E M \\
n=40\end{array}$ & $\begin{array}{l}\text { Error (\%) } \\
\text { for } n=5\end{array}$ \\
\hline$\omega_{1}$ & 11.4 & 11.4 & 0.17 \\
\hline$\omega_{2}$ & 65.8 & 66.4 & 0.79 \\
\hline$\omega_{3}$ & 70.4 & 71.7 & 1.94 \\
\hline
\end{tabular}

Table 4.13: The bending-bending-torsion natural frequencies

\begin{tabular}{cccc} 
of the pre-twisted cantilever rotating blade $\Omega=360 \mathrm{rpm}$ \\
\hline $\begin{array}{c}\text { Frequency } \\
(\mathrm{rad} / \mathrm{s})\end{array}$ & $\begin{array}{c}\text { Triple load path } \\
\text { TMM method [6] }\end{array}$ & $\begin{array}{c}\text { FEM } \\
n=40\end{array}$ & $\begin{array}{c}\text { Error }(\%) \\
\text { for } n=5\end{array}$ \\
\hline$\omega_{1}$ & 45.1 & 46.7 & 3.52 \\
$\omega_{2}$ & 72.3 & 73.7 & 1.91 \\
$\omega_{3}$ & 125.0 & 133.1 & 6.47 \\
\hline
\end{tabular}

One can see that the difference between triple load path and cantilevered configurations for the first few frequencies of non-rotating case is negligible, while adding rotating speed to the blade causes slightly different dynamic behaviour. Higher frequencies exhibit larger differences between cantilevered blade and triple load path blade configurations.

In order to study the effect of rotating speed on vibration frequencies and modes, the variation of the first 10 frequencies of the helicopter blade [6] (with cantilever boundary conditions) versus blade rotating speed is shown in Figure 4.10. 


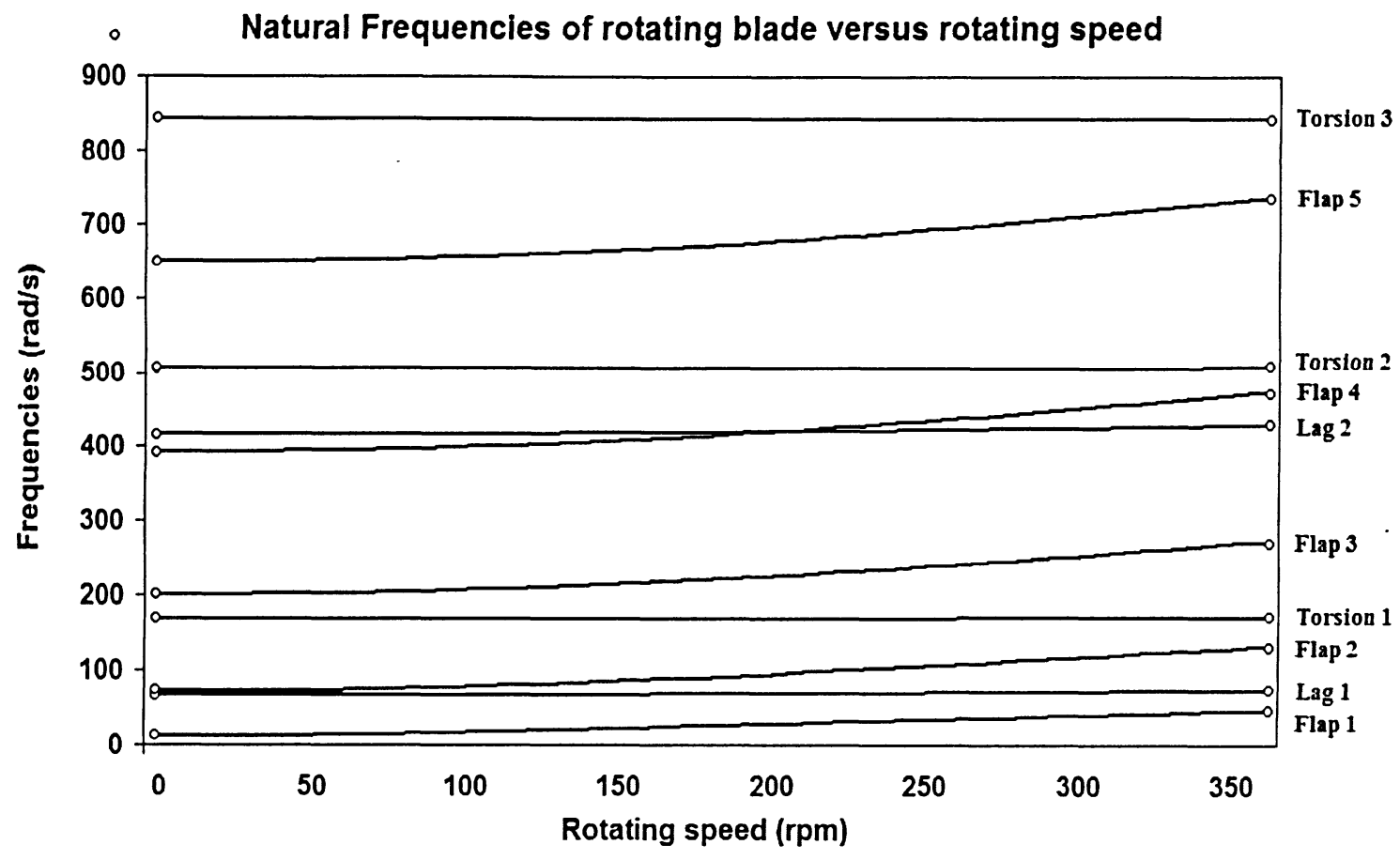

Figure 4.11: The effect of rotary speed on frequencies and modes of triply coupled rotating blade.

Figure 4.10 shows that, as expected, the rotating speed of beam can change the blade's natural frequencies. One can see that due to the centripetal effect, the stiffening effect of rotating speed for lag vibrations decreases. The flap frequencies increase as rotating speed increases, but the increments in lag frequencies are less pronounced. A closer look at "Flap 4" and "Lag 2" modes reveals that by increasing the rotating speed flap frequency passes the lag frequency. Besides, the torsion frequencies are almost constant. The vibrational behaviour of the cantilever blade shown in Figure 4.10 is very similar to real triple load path blade model [16].

All examples of this chapter verified the applicability of FEM method as it has already been used in last decades for analysis of many flexible structures including beams. The convergence rates of the FEM methodology introduced in this chapter are very good, and for preliminary design purposes and to get the few first frequencies and modes, a model of only 10 to 30 elements can be exploited. 
In next chapter, the DFE formulation based on Galerkin-type WRM will be discussed and the Dynamic Trigonometric Shape Functions (DTSF's) will be employed to solve the governing differential equations of motion of dually and triply coupled vibrations of beams and rotating blades. 


\section{CHAPTER 5: DYNAMIC FINITE ELEMENT METHOD}

\subsection{Introduction}

The Dynamic Finite Element (DFE) method is an intermediate formulation between exact DSM and classic FEM methods. The main difference between the classical FEM method and the DFE is "the definition of approximation functions". In the traditional FEM (Chapter 4) the cubic polynomial shape functions for flexural displacements and linear shape function for torsional displacement are used, where theses shape functions are the solutions of static deformation of a beam in elastic area. In the $\mathrm{DFE}$, the solutions of the differential equations governing the uncoupled vibrations of the beam are chosen as the basis functions of approximation space. The dynamic interpolation (shape) functions of approximation are then obtained based on the standard FEM approach.

\subsection{Frequency Dependent Approximation Functions}

In general, the DFE and FEM methods follow the same formulation that means the DFE approach starts with Galerkin weak formulation and integral form of equations. Then the same integration by parts are applied to the equations to satisfy the natural (force) boundary conditions which results in equations (4.10) to (4.12). The major difference between two methods is the basis functions from which the shape functions are calculated. As already mentioned, the basis functions in the DFE method are dynamic (frequency dependent) trigonometric shape functions (DTSF's). The basis functions are extracted from differential equations governing uncoupled vibrations of the beam. $\langle\boldsymbol{P}(\xi)\rangle_{f}$ and $\langle\boldsymbol{P}(\xi)\rangle_{t}$, the basis functions for flexural and torsional displacements, respectively, then can be defined as: 


$$
\begin{gathered}
<P(\xi)>_{f}=<\cos (\alpha \xi) ; \frac{\sin (\alpha \xi)}{\alpha} ; \frac{\cosh (\beta \xi)-\cos (\alpha \xi)}{\alpha^{2}+\beta^{2}} ; \frac{\sinh (\beta \xi)-\sin (\alpha \xi)}{\alpha^{3}+\beta^{3}}> \\
<P(\xi)>_{1}=<\cos (\tau \xi) \quad \frac{\sin (\tau \xi)}{\tau}>
\end{gathered}
$$

where $\alpha, \beta$ (separately calculated for $v$ and $w$ ) and $\tau$ can be obtained by solving the uncoupled equations of beam for bending and torsion vibrations (see Appendix A):

$$
\begin{gathered}
\alpha=\sqrt{\frac{\left|-B-\sqrt{B^{2}-4 A C}\right|}{2 A}} ; \beta=\sqrt{\frac{-B+\sqrt{B^{2}-4 A C}}{2 A}} \\
\tau=\omega l_{e} \sqrt{\frac{m \kappa_{m}{ }^{2}}{G J}} \\
A=\frac{E I}{l_{e}^{3}} ; B=-\frac{T}{l_{e}} ; C=-m l_{e} \omega^{2}
\end{gathered}
$$

The basis functions have been manipulated to reduce to Hermite and linear basis functions for bending and torsion, respectively, as $\alpha, \beta, \tau \rightarrow 0$. The Hermite basis functions have been widely used in finite elements for many years, for two main reasons, they satisfy completeness and continuity. Completeness is satisfied by including the lowest order admissible term. Continuity condition is met such that the shape functions are continuous across all inter-element boundaries. With these conditions satisfied the DFE with its Hermite based trigonometric basis functions is guaranteed to converge monotonically to the exact solution. The classic basis functions of approximation space for standard "Hermite" beam element (flexural displacements) are $1, \xi, \xi^{2}, \xi^{3}$ and the linear basis functions (torsional displacements) are $1, \xi$.

The so-called non-nodal approximation of displacements can then be written in terms of basis functions: 


$$
\begin{gathered}
w(\xi)=<P(\xi)>_{f-w}\left\{a_{w}\right\} \\
v(\xi)=<P(\xi)>_{f-v}\left\{a_{v}\right\} \\
\varphi(\xi)=<P(\xi)>_{t}\left\{a_{t}\right\}
\end{gathered}
$$

where the constant vectors $\left\{a_{w}\right\},\left\{a_{v}\right\}$ and $\left\{a_{t}\right\}$ can be calculated using the nodal displacements:

$$
\begin{gathered}
\left\{w_{n}\right\}=\left[P_{n}\right]_{f-w}\left\{a_{w}\right\} \\
\left\{v_{n}\right\}=\left[P_{n}\right]_{f, v}\left\{a_{v}\right\} \\
\left\{\varphi_{n}\right\}=\left[P_{n}\right]_{t}\left\{a_{t}\right\}
\end{gathered}
$$

where $\left\{\boldsymbol{w}_{n}\right\},\left\{\boldsymbol{v}_{n}\right\}$ and $\left\{\boldsymbol{\phi}_{\boldsymbol{n}}\right\}$ are the nodal displacements, and $\left[\boldsymbol{P}_{\boldsymbol{n}}\right]_{f-w / v}$ and $\left[\boldsymbol{P}_{\boldsymbol{n}}\right]_{t}$ are:

$$
\left[P_{n}\right]_{f-w / v}=\left[\begin{array}{cccc}
1 & 0 & 0 & 0 \\
0 & 1 & 0 & \frac{\beta_{w / v}-\alpha_{w / v}}{\alpha_{w / v}^{3}+\beta_{w / v}} \\
\cos \left(\alpha_{w / v}\right) & \frac{\sin \left(\alpha_{w / v}\right)}{\alpha_{w / v}} & \frac{\cosh \left(\beta_{w / v}\right)-\cos \left(\alpha_{w / v}\right)}{\alpha_{w / v}{ }^{2}+\beta_{\beta^{2}}{ }^{2}} & \frac{\sinh \left(\beta_{w / v}\right)-\sin \left(\alpha_{w / v}\right)}{\alpha_{w / v}{ }^{3}+\beta_{w / v}{ }^{3}} \\
-\alpha_{w / v} \sin \left(\alpha_{w / v}\right) & \cos \left(\alpha_{w / v}\right) & \frac{\beta_{w / v} \sinh \left(\beta_{w / v}\right)+\alpha_{w / v} \sin \left(\alpha_{w / v}\right)}{\alpha_{w / v}{ }^{2}+\beta_{w / v}{ }^{2}} & \frac{\beta_{w / v} \cosh \left(\beta_{w / v}\right)-\alpha_{w / v} \cos \left(\alpha_{w / v}\right)}{\alpha_{w / v}{ }^{3}+\beta_{w / v}}
\end{array}\right]
$$

and

$$
\left[P_{n}\right]_{t}=\left[\begin{array}{cc}
1 & 0 \\
-\frac{\cos (\tau) \tau}{\sin (\tau)} & \frac{\tau}{\sin (\tau)}
\end{array}\right]
$$


By determining the coefficient vectors $\left\{a_{w}\right\},\left\{a_{v}\right\}$ and $\left\{a_{t}\right\}$ in terms of nodal displacements, the element displacements can be written in terms of shape functions and nodal displacements (i.e., nodal approximations):

$$
\begin{gathered}
w(\xi)=<P(\xi)>_{f, w}\left[P_{n}\right]_{f, w}^{-1}\left\{w_{n}\right\}=<N_{B, w}(\xi)>\left\{w_{n}\right\} \\
v(\xi)=<P(\xi)>_{f, v}\left[P_{n}\right]_{f, v}^{-1}\left\{v_{n}\right\}=<N_{B, v}(\xi)>\left\{v_{n}\right\} \\
\varphi(\xi)=<P(\xi)>_{t}\left[P_{n}\right]_{t}^{-1}\left\{\varphi_{n}\right\}=<N_{T}(\xi)>\left\{\varphi_{n}\right\}
\end{gathered}
$$

where the bending and torsion shape functions are:

$$
\begin{aligned}
<N_{B}(\xi)>_{1}= & \frac{\alpha \beta}{D_{f}}\{-\cos (\alpha \xi)+\cos (\alpha(1-\xi)) \cosh (\beta)+\cos (\alpha) \cosh (\beta(1-\xi)) \\
- & \left.\cosh (\beta \xi)-\frac{\beta}{\alpha} \sin (\alpha(1-\xi)) \sinh (\beta)+\frac{\alpha}{\beta} \sin (\alpha) \sinh (\beta(1-\xi))\right\} \\
<N_{B}(\xi)>_{2}= & \frac{1}{D_{f}}\{\beta[\cosh (\beta(1-\xi)) \sin (\alpha)-\sin (\alpha(1-\xi)) \cosh (\beta)-\sin (\alpha \xi)] \\
& +\alpha[\cos (\alpha(1-\xi)) \sinh (\beta)-\cos (\alpha) \sinh (\beta(1-\xi))-\sinh (\beta \xi)]\} \\
<N_{B}(\xi)>_{3}= & \frac{\alpha \beta}{D_{f}}\{-\cos (\alpha(1-\xi))+\cos (\alpha \xi) \cosh (\beta)-\cosh (\beta(1-\xi)) \\
& \left.+\cos (\alpha) \cosh (\beta \xi)-\frac{\beta}{\alpha} \sin (\alpha \xi) \sinh (\beta)+\frac{\alpha}{\beta} \sin (\alpha) \sinh (\beta \xi)\right\} \\
<N_{B}(\xi)>_{4} & =\frac{1}{D_{f}}\{\beta[-\cosh (\beta(\xi) \sin (\alpha)-\sin (\alpha(1-\xi))+\sin (\alpha \xi) \cosh (\beta)] \\
& +\alpha[-\cos (\alpha \xi) \sinh (\beta)-\sinh (\beta(1-\xi))+\cos (\alpha) \sinh (\beta \xi)]\}
\end{aligned}
$$

and 


$$
\begin{aligned}
<N_{\tau}(\xi)>_{1} & =\frac{1}{D_{t}}\{\sin (\tau) \cos (\tau \xi)-\cos (\tau) \sin (\tau \xi)\} \\
& <N_{T}(\xi)>_{2}=\frac{1}{D_{t}} \sin (\tau \xi)
\end{aligned}
$$

The parameters $\boldsymbol{D}_{\boldsymbol{f}}$ and $\boldsymbol{D}_{\boldsymbol{t}}$ are:

$$
\begin{gathered}
D_{f}=\alpha \beta\left\{2[\cos (\alpha) \cosh (\beta)-1]+\frac{\alpha^{2}-\beta^{2}}{\alpha \beta} \sin (\alpha) \sinh (\beta)\right\} \\
D_{t}=\sin (\tau)
\end{gathered}
$$

where $\alpha$ and $\beta$ in equations (5.17) to (5.24) can be replaced by $\alpha_{w}$ and $\beta_{w}$ or $\alpha_{v}$ and $\beta_{v}$ to obtain the shape functions of out-of-plane or in-plane displacements, respectively.

The shape functions (5.17) to (5.22) are called Dynamic (frequency dependent) Trigonometric Shape Functions (DTSF). The bending shape functions have been shown in Figure 5.1 for the first four natural frequencies of a non-rotating beam with pure flap vibrations. 

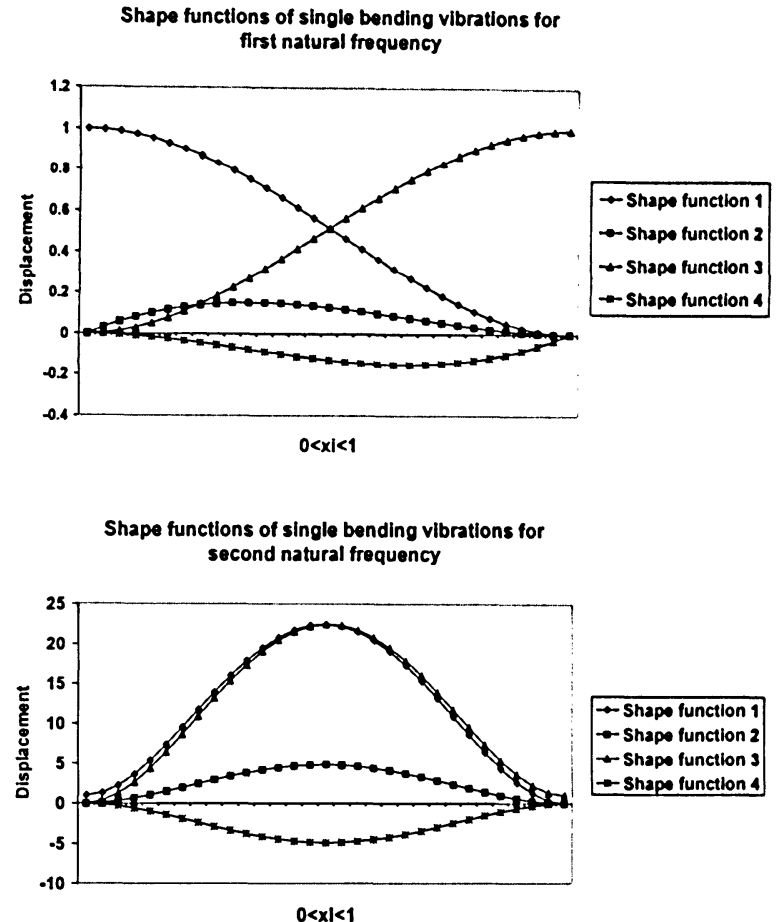

Shape functions of single bending vibrations for third natural frequency

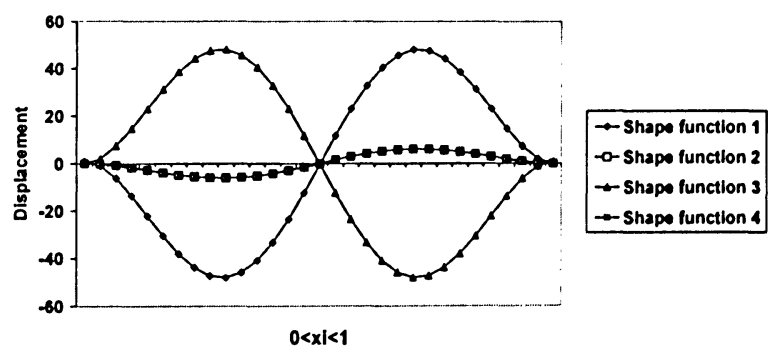

Shape functions of single bending vibrations for fourth natural frequency

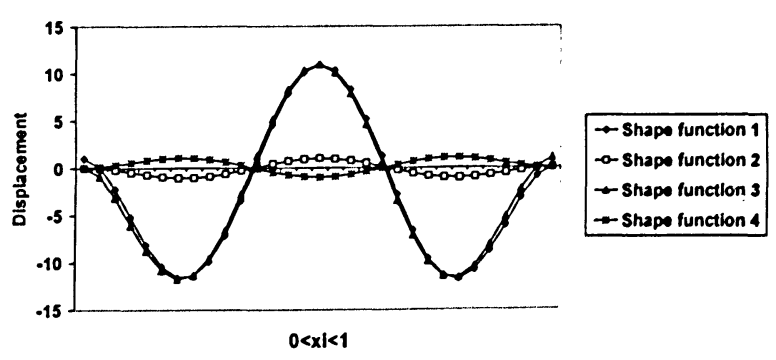

Figure 5.1: The beam bending shape functions calculated at its first four natural frequencies. 


\subsection{Derivation of System Eigenproblem}

Implementing the Galerkin-type Weighted Residual Method (WRM) on the governing differential equations (2.1) to (2.3) and applying the natural boundary conditions, the element virtual works (after discretization and performing appropriate integration by parts) is written in following form:

$$
\begin{aligned}
& W_{f, w}^{e}=\int_{0}^{+1} \underbrace{\left\{w^{n \prime \prime}\left(\frac{E I_{y}}{l^{3}}\right) w-\delta w^{\prime \prime}\left(\frac{T}{l}\right) w-\delta w\left(\omega^{2} m l\right) w\right\}}_{\left(^{*}\right)} d \xi \\
& +\int_{0}^{+1}\left\{\delta w^{\prime \prime}\left(\frac{E I_{z y}}{l^{3}}\right) v^{\prime \prime}+\delta w^{\prime}\left(\Omega^{2} e m\left(x+e_{1}\right) \cos \theta\right) \phi\right. \\
& \left.-\delta w\left(\omega^{2} e m l \cos \theta\right) \phi\right\} d \xi \\
& +\left[\delta w^{\prime \prime}\left(\frac{E I_{y}}{l^{3}}\right) w^{\prime}-\delta w^{\prime \prime \prime}\left(\frac{E I_{y}}{l^{3}}\right) w+\delta w^{\prime}\left(\frac{T}{l}\right) w\right]_{0}^{1} \\
& W_{f, v}^{e}=\int_{0}^{+1}\{\underbrace{\delta v^{\prime \prime \prime}\left(\frac{E I_{z}}{l^{3}}\right) v-\delta v^{\prime \prime}\left(\frac{T}{l}\right) v-\delta v\left(\omega^{2} m l\right) v}_{(\cdot \cdots)}\} d \xi \\
& +\int_{0}^{+1}\left\{\delta v^{\prime \prime}\left(\frac{E I_{z y}}{l^{3}}\right) w^{\prime \prime}-\delta v^{\prime}\left(\Omega^{2} e m\left(x+e_{1}\right) \sin \theta\right) \phi\right. \\
& \left.\left.+\delta v\left[\left(\Omega^{2}+\omega^{2}\right) e m l \sin \theta\right)\right] \phi-\delta v\left(\Omega^{2} m l\right) v\right\} d \xi \\
& +\left[\delta v^{\prime \prime}\left(\frac{E I_{z}}{l^{3}}\right) v^{\prime}-\delta v^{\prime \prime \prime}\left(\frac{E I_{z}}{l^{3}}\right) v+\delta v^{\prime}\left(\frac{T}{l}\right) v\right]_{0}^{1} \\
& W_{t, \phi}^{e}=\int_{0}^{+1} \underbrace{\left\{-\delta \phi^{\prime \prime}\left(\frac{G J}{l}\right) \phi-\delta \phi\left(\omega^{2} m l \kappa_{m}^{2}\right) \phi\right\}}_{(\because)} d \xi \\
& +\int_{0}^{+1}\left\{\delta \phi\left(\Omega^{2} e m\left(x+e_{1}\right) \cos \theta\right) w^{\prime}-\delta \phi\left(\Omega^{2} e m\left(x+e_{1}\right) \sin \theta\right) v^{\prime}\right. \\
& \left.+\delta \phi\left[\left(\Omega^{2}+\omega^{2}\right) e m l \sin \theta\right)\right] v-\delta \phi\left(\omega^{2} e m l \cos \theta\right) w \\
& \left.+\delta \phi\left(\Omega^{2} m l\left(\kappa_{m 2}^{2}-\kappa_{m 1}^{2}\right) \cos 2 \theta\right) \phi\right\} d \xi \\
& +\left[\delta \phi^{\prime}\left(\frac{G J}{l}\right) \phi\right]_{0}^{1}
\end{aligned}
$$


where $0 \leq \xi \leq 1$. The approximations for $w, \delta w, v, \delta v, \phi$ and $\delta \phi$ are made such that the integral terms $\left({ }^{*}\right),\left({ }^{* *}\right)$ and $\left({ }^{* * *}\right)$ in the above equations $(5.25)$ to $(5.27)$ vanish, or:

$$
\begin{gathered}
{\left[\delta w^{\prime \prime \prime}\left(\frac{E I_{y}}{l^{3}}\right)-\delta w^{\prime \prime}\left(\frac{T}{l}\right)-\delta i v\left(\omega^{2} m l\right)\right] w=0} \\
{\left[\delta v^{\prime \prime \prime}\left(\frac{E I_{z}}{l^{3}}\right)-\delta v^{\prime \prime}\left(\frac{T}{l}\right)-\delta v\left(\omega^{2} m l\right)\right] v=0} \\
{\left[\delta \phi^{\prime \prime}\left(\frac{G J}{l}\right)+\delta \phi\left(\omega^{2} m l \kappa_{m}^{2}\right)\right] \phi=0}
\end{gathered}
$$

The equations (5.28) to (5.30) can be used to determine the parameters $\alpha_{w}, \beta_{w}, \alpha_{v}$, $\beta_{v}$ and $\tau$ following the approaches introduced in Appendix A. The components of element virtual work then reduce to the following forms:

$$
\begin{aligned}
& W_{f, w}^{e}= \int_{0}^{+1}\left\{\delta w^{\prime \prime}\left(\frac{E I_{z y}}{l^{3}}\right) v^{\prime \prime}+\delta w^{\prime}\left(\Omega^{2} e m\left(x+e_{1}\right) \cos \theta\right) \phi\right. \\
&\left.-\delta w\left(\omega^{2} e m l \cos \theta\right) \phi\right\} d \xi \\
&+\left[\delta w^{\prime \prime}\left(\frac{E I_{y}}{l^{3}}\right) w^{\prime}-\delta w^{\prime \prime \prime}\left(\frac{E I_{y}}{l^{3}}\right) w+\delta w^{\prime}\left(\frac{T}{l}\right) w\right]_{0}^{1} \\
& W_{f, v}^{e}=\int_{0}^{+1}\left\{\delta v^{\prime \prime}\left(\frac{E I_{z y}}{l^{3}}\right) w^{\prime \prime}-\delta v^{\prime}\left(\Omega^{2} e m\left(x+e_{1}\right) \sin \theta\right) \phi\right. \\
&+\left.\left.\delta v\left[\left(\Omega^{2}+\omega^{2}\right) e m l \sin \theta\right)\right] \phi-\delta v\left(\Omega^{2} m l\right) v\right\} d \xi \\
&+\left[\delta v^{\prime \prime}\left(\frac{E I_{z}}{l^{3}}\right) v^{\prime}-\delta v^{\prime \prime \prime}\left(\frac{E I_{z}}{l^{3}}\right) v+\delta v^{\prime}\left(\frac{T}{l}\right) v\right]_{0}^{1} \\
& W_{t, \phi}^{e}=\int_{0}^{+1}\left\{\delta \phi\left(\Omega^{2} e m\left(x+e_{1}\right) \cos \theta\right) w^{\prime}-\delta \phi\left(\Omega^{2} e m\left(x+e_{1}\right) \sin \theta\right) v^{\prime}\right. \\
&+\left.\delta \phi\left[\left(\Omega^{2}+\omega^{2}\right) e m l \sin \theta\right)\right] v-\delta \phi\left(\omega^{2} e m l \cos \theta\right) w \\
&+\left.\delta \phi\left(\Omega^{2} m l\left(\kappa_{m 2}^{2}-\kappa_{m 1}^{2}\right) \cos 2 \theta\right) \phi\right\} d \xi \\
&+ {\left[\delta \phi^{\prime}\left(\frac{G J}{l}\right) \phi\right]_{0}^{1} }
\end{aligned}
$$


and the element virtual work can be written as:

$$
W_{\mathrm{int}}^{e}=<\delta u>^{e}[K(\omega)]^{e}\{u\}^{e}
$$

where the element nonlinear dynamic (frequency dependent) stiffness matrix, $[K(\omega)]^{e}$, can be written as:

$$
[K(\omega)]^{e}=[K(\omega)]_{\text {Uncoupled }}^{e}+[K(\omega)]_{\text {Coupled }}^{e}
$$

The coupled and uncoupled element stiffness matrices are extracted from equations (5.31), (5.32) and (5.33) considering the nodal parameters of equations (4.21) to (4.24):

$$
\begin{aligned}
& {[K(\omega)]_{\text {Uncoupled }}^{e}=} \\
& {[\overbrace{c_{w}\left\{N_{B-w}^{m}\right\}-c_{T}\left\{N_{B-w}^{\prime}\right\} ;-c_{w}\left\{N_{B-w}^{n}\right\} ; c_{v}\left\{N_{B-v}^{m}\right\}-c_{T}\left\{N_{B-v}^{\prime}\right\} ;-c_{v}\left\{N_{B-v}^{n}\right\} ;-c_{\varphi}\left\{N_{T-\varphi}^{\prime}\right\}}^{\xi=0} ;} \\
& \overbrace{c_{T}\left\{N_{B-w}^{\prime}\right\}-c_{w}\left\{N_{B-w}^{m}\right\} ; c_{w}\left\{N_{B-w}^{n}\right\} ; c_{T}\left\{N_{B-v}^{\prime}\right\}-c_{v}\left\{N_{B-v}^{m}\right\} ; c_{v}\left\{N_{B-v}^{n}\right\} ; c_{\varphi}\left\{N_{T-\varphi}^{\prime}\right\}}^{\xi=1}] \\
& +\int_{0}^{+1}\left[\left(\Omega^{2} m l\left(\kappa_{m 2}^{2}-\kappa_{m 1}^{2}\right) \cos 2 \theta\right)\left\{N_{T-\varphi}\right\}<N_{T-\phi}>+\left(-\Omega^{2} m l\right)\left\{N_{B-\nu}\right\}<N_{B-\nu}>\right] d \xi \\
& {[K(\omega)]_{\text {Coupled }}^{e}=\int_{0}^{+1}\left[\left(\frac{E I_{z y}}{l^{3}}\right)\left(\left\{N_{B-w}^{n}\right\}<N_{B-v}^{n}>+\left\{N_{B-v}^{n}\right\}<N_{B-w}^{n}>\right)\right.} \\
& -\left(\omega^{2} e m l \cos \theta\right)\left(\left\{N_{B-w}\right\}<N_{T-\varphi}>+\left\{N_{T-\varphi}\right\}<N_{B-w}>\right) \\
& +\left(\Omega^{2} e m\left(x+e_{1}\right) \cos \theta\right)\left(\left\{N_{B-w}^{\prime}\right\}<N_{T-\varphi}>+\left\{N_{T-\varphi}\right\}<N_{B-w}^{\prime}>\right) \\
& \left.+\left[\left(\Omega^{2}+\omega^{2}\right) e m l \sin \theta\right)\right]\left(\left\{N_{B-\nu}\right\}<N_{T-\varphi}>+\left\{N_{T-\phi}\right\}<N_{B-v}>\right) \\
& \left.-\left(\Omega^{2} e m\left(x+e_{1}\right) \sin \theta\right)\left(\left\{N_{B-\nu}^{\prime}\right\}<N_{T-\varphi}>-\left\{N_{T-\phi}\right\}<N_{B-\nu}^{\prime}>\right)\right] d \xi
\end{aligned}
$$

where: 


$$
c_{w}=\frac{E I_{y}}{l^{3}} ; c_{v}=\frac{E I_{z}}{l^{3}} ; c_{\varphi}=\frac{G J}{l} ; c_{T}=\frac{T}{l}
$$

The system equation then can be obtained by the standard assembly process:

$$
W_{\mathrm{int}}=\sum_{1}^{N E} W_{\mathrm{int}}^{e}=0
$$

or

$$
W_{\text {int }}=<\delta u>[K(\omega)]\{u\}=0
$$

where:

$$
[K(\omega)]=\sum_{1}^{N E}[K(\omega)]^{e}
$$

The virtual displacements vector $\langle\delta \boldsymbol{u}>$ in equation (5.40) is arbitrary, then the rest of equation should vanish:

$$
[K(\omega)]\{u\}=0
$$

The stiffness matrix is frequency dependent and the equation (5.42) represents a nonlinear eigenproblem. The frequencies of this eigenproblem are calculated by WittrickWilliams root counting algorithm $[14,15,26]$ introduced in Chapter 3.

\subsection{Refined Dynamic Finite Element (RDFE)}

The parameters $E I_{y}, E I_{z}, T, m, G J$ and $m \kappa_{m}{ }^{2}$ in equations (5.28) to (5.30) are assumed to be constant inside each element domain. If the beam specifications vary along the length of the beam, this assumption is not valid and the equations (5.28) to (5.30) 
cannot result exactly the assumed basis functions. For small number of elements, where the variation of parameters are considerably large, vanishing $(*),(* *)$ and $\left({ }^{* * *}\right)$ in equations (5.25), (5.26) and (5.27) results some errors. In order to avoid this error a compensating term can be defined for each variable parameter to be added to the equations, called deviator term. The variable parameters can be written as:

$$
\begin{gathered}
E I(\xi)=E I+E I_{D E V} \\
T(\xi)=T+T_{D E V} \\
m(\xi)=m+m_{D E V} \\
G J(\xi)=G J+G J_{D E V} \\
m \kappa_{m}^{2}(\xi)=m \kappa_{m}^{2}+\left(m \kappa_{m}^{2}\right)_{D E V}
\end{gathered}
$$

where $E I, T, \boldsymbol{m}, \boldsymbol{G J}$ and $\boldsymbol{m} \boldsymbol{\kappa}_{\boldsymbol{m}}{ }^{2}$ are average values in each element, and the parameters with subscript " $\boldsymbol{D E} V$ " are the deviation of average values from exact values [21]. The uncoupled stiffness matrix then can be rearranged as:

$$
\begin{aligned}
& {[K(\omega)]_{\text {Uncoupled }}^{e}=} \\
& \overbrace{\left[c_{w}\left\{N_{B-w}^{m}\right\}-c_{T}\left\{N_{B-w}^{\prime}\right\} ;-c_{w}\left\{N_{B-w}^{n}\right\} ; c_{v}\left\{N_{B-\nu}^{m}\right\}-c_{T}\left\{N_{B-\nu}^{\prime}\right\} ;-c_{\nu}\left\{N_{B-\nu}^{n}\right\} ;-c_{\varphi}\left\{N_{T-\phi}^{\prime}\right\}\right.}^{\xi=0} ; \\
& \overbrace{c_{T}\left\{N_{B-w}^{\prime}\right\}-c_{w}\left\{N_{B-w}^{m}\right\} ; c_{w}\left\{N_{B-w}^{n}\right\} ; c_{T}\left\{N_{B-v}^{\prime}\right\}-c_{v}\left\{N_{B-v}^{m}\right\} ; c_{v}\left\{N_{B-v}^{\prime}\right\} ; c_{\varphi}\left\{N_{T-\varphi}^{\prime}\right\}}^{\xi=1}] \\
& +\int_{0}^{+1}\left[\left(\Omega^{2} m l\left(\kappa_{m 2}^{2}-\kappa_{m l}^{2}\right) \cos 2 \theta\right)\left\{N_{T-\phi}\right\}<N_{T-\varphi}>+\left(-\Omega^{2} m l\right)\left\{N_{B-v}\right\}<N_{B-v}>\right] d \xi \\
& +D E V
\end{aligned}
$$

where: 


$$
\begin{aligned}
D E V= & \int^{+1}\left[\left(\frac{E I_{y}(\xi)-E I_{y}}{l^{3}}\right)\left\{N_{B-w}^{\prime \prime}\right\}<N_{B-w}^{*}>+\left(\frac{E I_{z}(\xi)-E I_{z}}{l^{3}}\right)\left\{N_{B-v}^{*}\right\}<N_{B-v}^{\prime \prime}>\right. \\
& -\left(\frac{T(\xi)-T}{l}\right)\left(\left\{N_{B-w}^{\prime}\right\}<N_{B-w}^{\prime}>+\left\{N_{B-v}^{\prime}\right\}<N_{B-v}^{\prime}>\right) \\
& -l \omega^{2}[m(\xi)-m]\left(\left\{N_{B-w}\right\}<N_{B-w}>+\left\{N_{B-v}\right\}<N_{B-v}>\right) \\
& \left.+\left(\frac{G J(\xi)-G J}{l}\right)\left\{N_{T-\varphi}^{\prime}\right\}<N_{T-\varphi}^{\prime}>-l \omega^{2}\left[m \kappa_{m}^{2}(\xi)-m \kappa_{m}^{2}\right]\left\{N_{T-\phi}\right\}<N_{T-\varphi}>\right] d \xi
\end{aligned}
$$

Adding $D E V$ matrix drastically increases the accuracy of the model and provides

faster convergence. It can be seen from equation (5.45) if any parameter in beam specification is constant, the corresponding term will not appear in DEV matrix. For uniform rotating beam all geometrical deviator terms vanish and the deviator matrix reduces to following form in which the effect of variable axial force, centrifugal force, is taken into account:

$$
D E V=\int_{0}^{+1}\left[-\frac{T(\xi)-T}{l}\left(\left\{N_{B-w}^{\prime}\right\}<N_{B-w}^{\prime}>+\left\{N_{B-v}^{\prime}\right\}<N_{B-v}^{\prime}>\right)\right] d \xi
$$

As it will be discussed later, without considering the deviator matrix, the convergence rate of DFE and FEM are nearly the same, but by "refining" the DFE element equations, the DFE convergence rates are effectively improved. The methodology presented here is called Refined DFE and will be used later in numerical examples where variable parameters and rotating speed are to be considered.

\subsection{Numerical Results}

The DFE method has already been applied to many bending-torsion coupled vibration problems including axially loaded or materially coupled composite beams [21-25]. In this thesis, some illustrative numerical examples are discussed to verify the DFE formulation introduced in this chapter. The natural frequencies, mode shapes and 
the convergence rate of DFE is investigated and compared with the existing results in literature and those presented in Chapter 4.

\subsubsection{Bending Vibrations of Uncoupled Beam}

As the first step, the natural frequencies and mode shapes of lateral vibrations of a non-rotating uniform beam is investigated. To implement the DFE, the integration by parts is applied to (4.41) and final equation will be in following form:

$$
W_{f, w}^{c}=\left[\delta w^{\prime \prime}\left(E I_{y}\right) w^{\prime}-\delta w^{m}\left(E I_{y}\right) w\right]_{x_{j}}^{x_{j+1}}
$$

and the uncoupled stiffness matrix, equation (5.36), reduces to the following form:

$$
[K(\omega)]_{\text {Uncoupled }}^{e}=[\overbrace{c_{w}\left\{N_{B-w}^{m}\right\},-c_{w}\left\{N_{B-w}^{n}\right\}}^{\xi=0}, \overbrace{-c_{w}\left\{N_{B-w}^{m}\right\}, c_{w}\left\{N_{B-,}^{n},\right.}^{\xi=1}]
$$

Using the Wittrick-Williams (W-W) root counting algorithm $[14,15,26]$, the natural frequencies can be evaluated from a one-element model. The first five frequencies of a uniform cantilever beam are shown in Table 5.1. The beam parameters are considered to be unit, $E I_{\boldsymbol{y}}=\boldsymbol{m}=\boldsymbol{L}=1$. As already stated, the DFE formulation for uncoupled bending is equivalent to the exact DSM method, due to the fact that the basis functions are the solutions of uncoupled bending vibrations. That is the reason why using a one-element mesh results in the exact frequencies. 
Table 5.1: The first five natural frequencies of out-of-plane vibration of a cantilever beam calculated by DFE

\begin{tabular}{cccc}
\hline $\begin{array}{c}\text { Natural } \\
\text { Frequency } \\
\text { (rad/s) }\end{array}$ & $\begin{array}{c}\text { Exact } \\
\text { analytical value } \\
{[34]}\end{array}$ & $\begin{array}{c}\text { DFE } \\
\text { With 1 elements }\end{array}$ & Error(\%) \\
\hline$\omega_{1}$ & 3.51602 & 3.51602 & 0.000 \\
$\omega_{2}$ & 22.0345 & 22.0345 & 0.000 \\
$\omega_{3}$ & 61.6972 & 61.6972 & 0.000 \\
$\omega_{4}$ & 120.9019 & 120.9019 & 0.000 \\
$\omega_{5}$ & 199.8595 & 199.8593 & 0.000 \\
\hline
\end{tabular}

The first three mode of vibration were also calculated by the DFE method and are plotted in Figure 5.2.

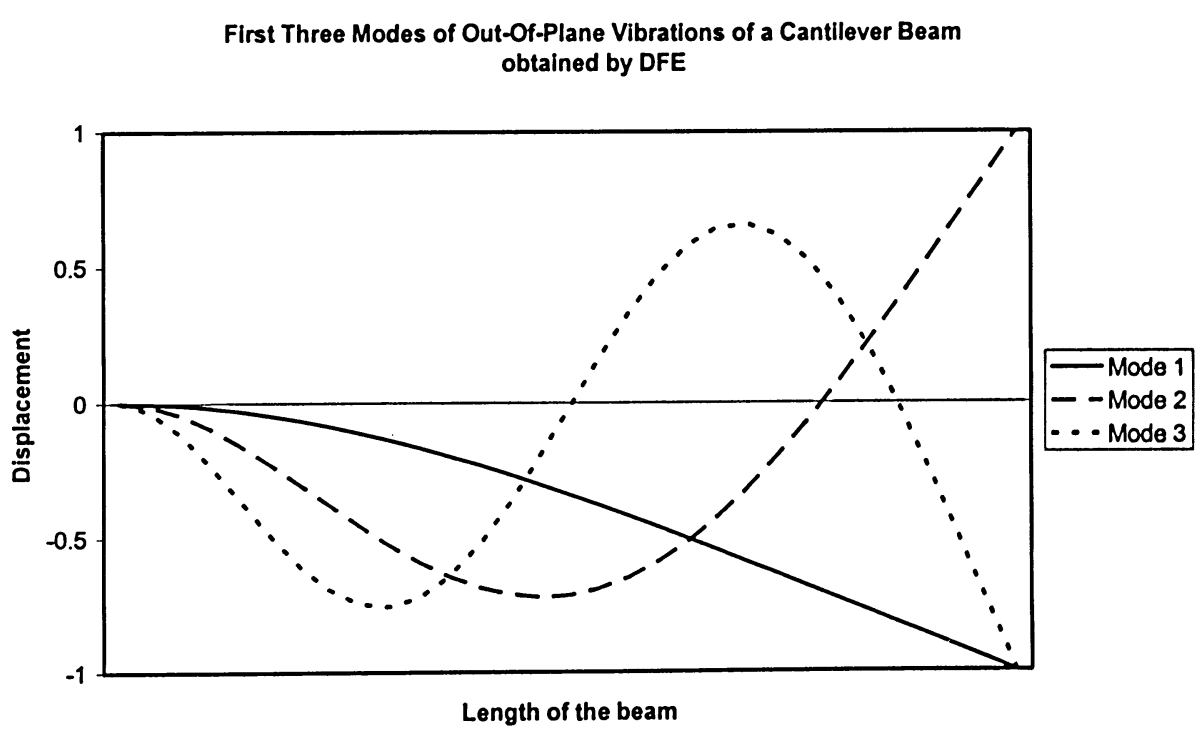

Figure 5.2: The first three out-of-plane modes of vibrations of a cantilever beam based on DFE method

From Table 5.1 and Figure 5.2, one can see that the natural frequencies and mode shapes confirm the validity and complete agreement of the DFE method and exact results for pure bending vibrations of a cantilever beam. 


\subsubsection{Coupled Bending-Torsion Vibrations}

The DFE analysis of coupled bending-torsion vibrations of beams for uniform beams as well as non-uniform composite beams, with or without axial forces has been well established [21-25]. In this section, some illustrative examples are studied to confirm the correctness of the presented DFE formulation.

\subsubsection{Non-rotating Beam:}

As the first coupled bending-torsion case, the dynamic behaviour of untwisted uniform coupled beams is studied. The equations of motion, in this case, can be extracted from (2.18) and (2.19). The element stiffness matrix, equations (5.35), is then written as:

$$
[K(\omega)]^{e}=[K(\omega)]_{\text {Uncoupled }}^{e}+[K(\omega)]_{\text {Coupled }}^{e}
$$

where

$$
[K(\omega)]_{\text {Uncoupled }}^{e}=[\overbrace{c_{w}\left\{N_{B-w}^{m}\right\} ;-c_{w}\left\{N_{B-w}^{n}\right\} ;-c_{\varphi}\left\{N_{T-\phi}^{\prime}\right\}}^{\xi=0} ; \overbrace{-c_{w}\left\{N_{B-w}^{m}\right\} ; c_{w}\left\{N_{B-w}^{n}\right\} ; c_{\varphi}\left\{N_{T-\varphi}^{\prime}\right\}}^{\xi=1}]
$$

and

$$
[K(\omega)]_{\text {Coupled }}^{e}=\int_{0}^{+1}\left[-\left(\omega^{2} e m l\right)\left(\left\{N_{B-w}\right\}<N_{T-\varphi}>+\left\{N_{T-\varphi}\right\}<N_{B-w}>\right)\right] d \xi
$$

Here, $\left\{N_{B-w}\right\}$ and $\left\{N_{T-\varphi}\right\}$ are the bending and torsional shape functions. A uniform cantilever wing [22] with following mechanical properties is considered: 


$$
\begin{aligned}
& L=6.0 \mathrm{~m} \\
& e=0.18 m \\
& \boldsymbol{m}=35.75 \mathrm{~kg} / \mathrm{m} \\
& E I=9.75 M N . m^{2} \\
& \boldsymbol{G} \boldsymbol{J}=0.988 M N . m^{2} \\
& \text { m. }_{\boldsymbol{m}_{m}}{ }^{2}=8.65 \mathrm{~kg} . \mathrm{m}
\end{aligned}
$$

The natural frequencies obtained from the DFE and FEM formulations along with those obtained from the exact DSM method [22] are presented in Table 5.2.

Table 5.2: The first six natural frequencies of coupled bending-torsion vibrations of a cantilever beam

\begin{tabular}{cccccc}
\hline $\begin{array}{c}\text { Natural } \\
\text { Frequency } \\
\text { (rad/s) }\end{array}$ & $\begin{array}{c}\text { Exact } \\
\text { Method } \\
(D S M)\end{array}$ & $\begin{array}{c}\text { DFE method } \\
\text { With } \\
5 \text { element }\end{array}$ & $\begin{array}{c}\text { Error of } \\
\text { DFE } \\
\text { method } \\
(\%)\end{array}$ & $\begin{array}{c}\text { FEM } \\
\text { element } \\
\text { With 30 } \\
\text { elements }\end{array}$ & $\begin{array}{c}\text { Error of FEM } \\
\text { 30-element } \\
(\%)\end{array}$ \\
\hline$\omega_{1}$ & 49.62 & 49.62 & 0.00 & 49.62 & 0.01 \\
$\omega_{2}$ & 97.04 & 97.05 & 0.01 & 97.05 & 0.01 \\
$\omega_{3}$ & 248.87 & 249.00 & 0.05 & 249.07 & 0.08 \\
$\omega_{4}$ & 355.59 & 357.54 & 0.55 & 355.78 & 0.05 \\
$\omega_{5}$ & 451.46 & 452.57 & 0.24 & 452.58 & 0.25 \\
$\omega_{6}$ & 610.32 & 610.63 & 0.05 & 613.59 & 0.54 \\
\hline
\end{tabular}

As it can be seen (Table 5.2), the comparison between higher modes obtained from the FEM and DFE (for example the fifth and sixth frequencies) shows the DFE's higher convergence rates.

A three-beam piecewise uniform wing, introduced in Table 4.3 and Figure 4.3, was also studied. The vibration analysis shows that DFE has better convergence rate than FEM, as a 6-element DFE model leads to the same results as a 15-element FEM model (Table 5.3). 
Table 5.3: The first five natural frequencies

of the three-beam aircraft wing obtained by DFE

\begin{tabular}{cccccc}
\hline $\begin{array}{c}\text { Frequency } \\
(\mathrm{rad} / \mathrm{s})\end{array}$ & $\begin{array}{c}\text { Exact Method } \\
(D S M)\end{array}$ & $\begin{array}{c}\text { DFE } \\
n=6\end{array}$ & $\begin{array}{c}\text { Error of DFE } \\
(\%)\end{array}$ & $\begin{array}{c}\text { FEM } \\
n=15\end{array}$ & $\begin{array}{c}\text { Error of FEM } \\
(\%)\end{array}$ \\
\hline$\omega_{1}$ & 74.43 & 74.44 & 0.02 & 74.43 & 0.01 \\
$\omega_{2}$ & 128.57 & 128.59 & 0.01 & 128.58 & 0.06 \\
$\omega_{3}$ & 253.40 & 253.44 & 0.01 & 253.56 & 0.32 \\
$\omega_{4}$ & 376.59 & 378.36 & 0.47 & 376.96 & 0.37 \\
$\omega_{5}$ & 431.29 & 433.42 & 0.49 & 431.97 & 0.67 \\
\hline
\end{tabular}

As another example, the DFE method was also implemented to the beam of following properties $[2,16]$ :

$$
\begin{aligned}
& \boldsymbol{L}=40.0 \mathrm{in} \\
& \boldsymbol{e}=0.4 \mathrm{in} \\
& \boldsymbol{m}=0.0015 \mathrm{slug} / \mathrm{in} \\
& \boldsymbol{k}_{\boldsymbol{m} I^{2}}=0.18 \mathrm{in}^{2} \\
& \boldsymbol{k}_{\boldsymbol{m} 2}{ }^{2}=0.71 \mathrm{in}^{2} \\
& E I=25000 \mathrm{lb}_{\mathrm{in}}{ }^{2} \\
& G J=9000 \mathrm{lb}_{\mathrm{in}}{ }^{2}
\end{aligned}
$$

The first five natural frequencies are calculated by DFE method and have been compared with Integrating Matrix Method [16] and the FEM (Chapter 4) as the reference values (see Table 5.4). 
Table 5.4: The bending-torsion coupled natural frequencies of the clamped-free beam

\begin{tabular}{cccccc}
\hline $\begin{array}{c}\text { Natural } \\
\text { Frequency } \\
\text { (rad/s) }\end{array}$ & $\begin{array}{c}\text { IMM } \\
\text { Method } \\
\text { (15 stations) }\end{array}$ & $\begin{array}{c}\text { DFE method } \\
\text { With } \\
5 \text { element }\end{array}$ & $\begin{array}{c}\text { Error of DFE } \\
\text { method } \\
\text { (\%) }\end{array}$ & $\begin{array}{c}\text { FEM element } \\
\text { With } \\
30 \text { elements }\end{array}$ & $\begin{array}{c}\text { Error of FEM } \\
\text { method } \\
(\%)\end{array}$ \\
\hline$\omega_{1}$ & 31.05 & 31.06 & 0.02 & 31.059 & 0.03 \\
$\omega_{2}$ & 193.74 & 193.79 & 0.02 & 193.748 & 0.00 \\
$\omega_{3}$ & 390.87 & 391.15 & 0.07 & 390.923 & 0.01 \\
$\omega_{4}$ & 539.54 & 540.34 & 0.15 & 539.598 & 0.01 \\
$\omega_{5}$ & 1043.94 & 1048.97 & 0.48 & 1044.306 & 0.04 \\
\hline
\end{tabular}

The DFE convergence rates for the first five natural frequencies are shown in Figure 5.3. Figure 5.4 presents the comparison between the convergence rates of DFE and FEM for the third and fifth frequencies to highlight the difference between DFE and FEM in higher modes.

Convergence test of DFE for bending-torsion vibrations

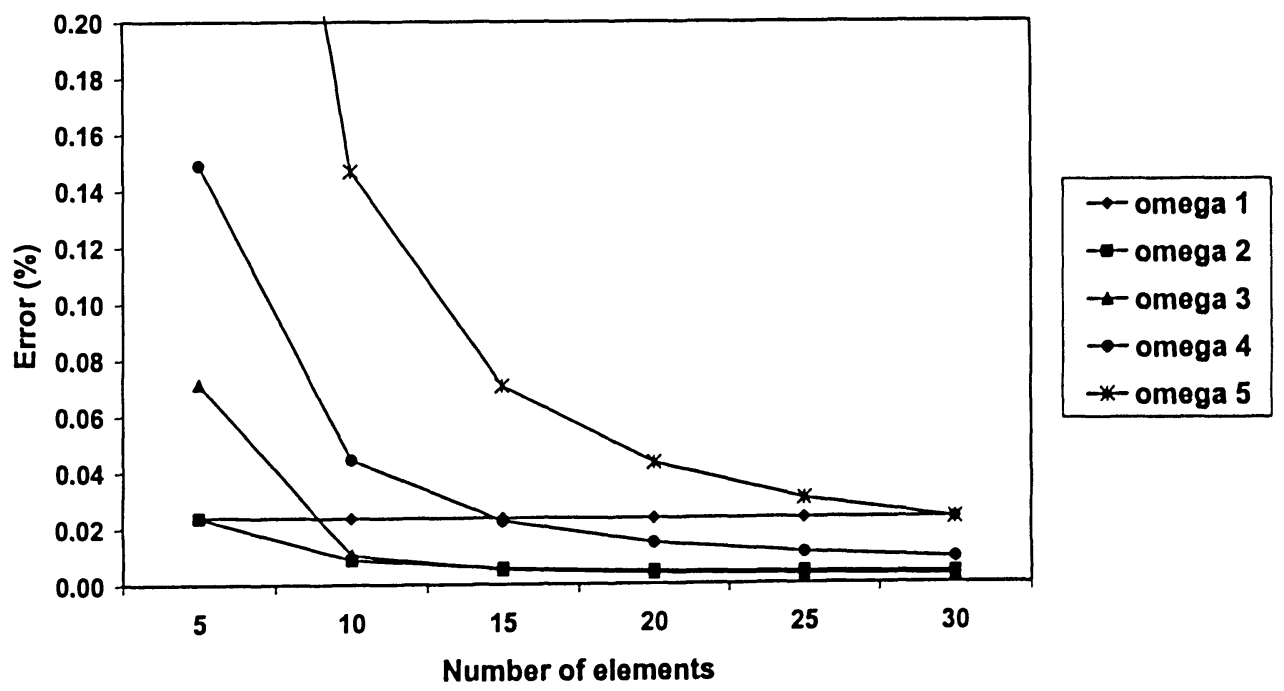

Figure 5.3: The DFE method convergence results for coupled bending-torsion vibrations. 


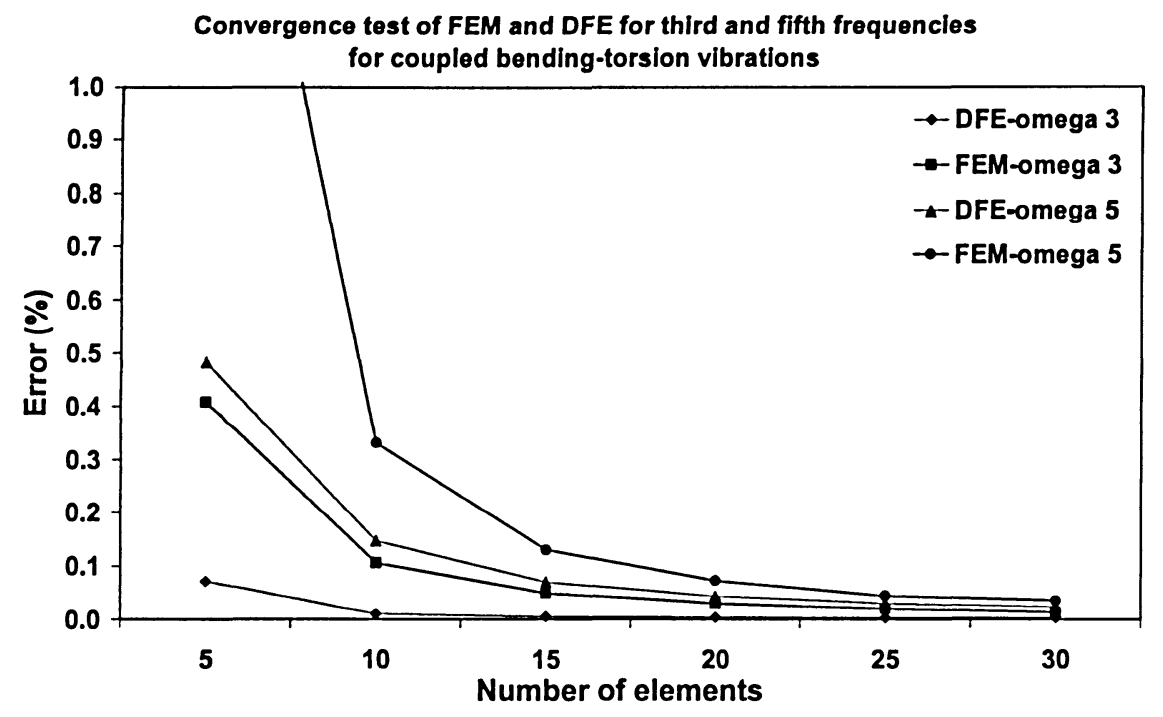

Figure 5.4: The comparison between the DFE and FEM convergence for coupled bending-torsion free vibrations.

The mode shapes of flap and torsion vibrations of the beam have been calculated and shown in Figures 5.5 and 5.6.

\section{Mode shapes of out-of-plane vibrations of bending-torsion coupled beam}

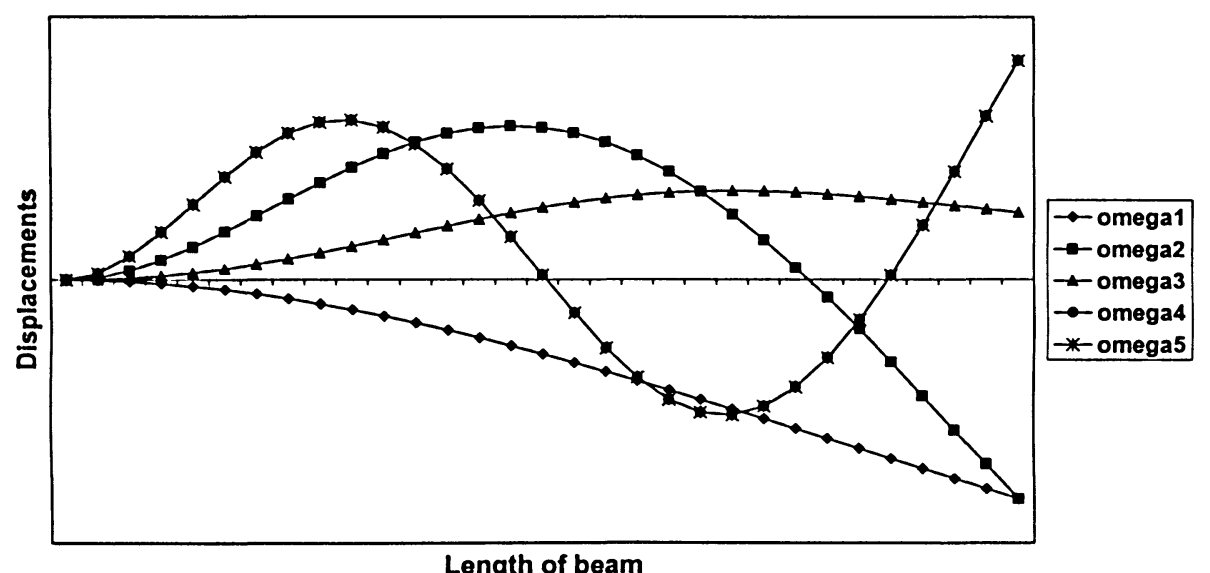

Figure 5.5: The DFE results for the first five modes of coupled bending-torsion vibrations for a cantilever beam; flap (bending) displacements. 


\section{First five modes of torsional vibrations}

of bending-torsion coupled beam

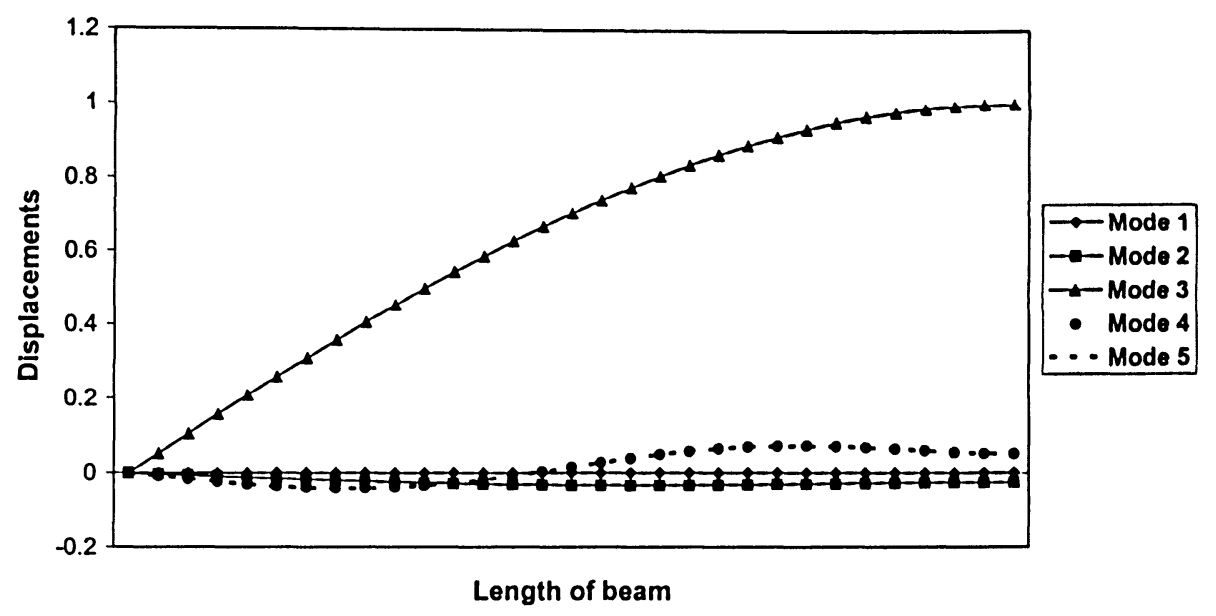

Figure 5.6: The DFE results for the first five modes of coupled bending-torsion vibrations for a cantilever beam; torsional displacements.

The examples of this section show that the results obtained by the DFE are in complete agreement with the results of the FEM (Chapter 4) and those of literature. Also the mode shapes calculated by the DFE exhibit the expected behaviour of the coupled bending-torsion vibrations of the non-rotating beams.

\subsubsection{Axially Loaded Coupled Beam:}

Considering the equations of motion introduced in expressions (4.48) and (4.49) [23], the element stiffness matrix for an axially loaded coupled bending-torsion beam can be written in following form:

$$
[K(\omega)]^{e}=[K(\omega)]_{\text {Uncoupled }}^{e}+[K(\omega)]_{\text {Coupled }}^{e}
$$

where 


$$
\begin{aligned}
& {[K(\omega)]_{\text {Uncoupled }}^{e}=} \\
& \overbrace{\left[c_{w}\left\{N_{B-w}^{m}\right\}-c_{T}\left\{N_{B-w}^{\prime}\right\} ;-c_{w}\left\{N_{B-w}^{\prime \prime}\right\} ;-c_{\varphi}\left\{N_{T-\varphi}^{\prime}\right\}\right.}^{\xi=0} ; \overbrace{c_{T}\left\{N_{B-w}^{\prime}\right\}-c_{w}\left\{N_{B-w}^{m}\right\} ; c_{w}\left\{N_{B-w}^{\prime \prime}\right\} ; c_{\varphi}\left\{N_{T-\varphi}^{\prime}\right\}}^{\xi=1}]
\end{aligned}
$$

and

$$
\begin{aligned}
& {[K(\omega)]_{\text {Coupled }}^{e}=\int_{0}^{+1} } {\left[-\left(\frac{T e}{l}\right)\left(\left\{N_{B-w}^{\prime}\right\}<N_{T-\varphi}^{\prime}>+\left\{N_{T-\varphi}^{\prime}\right\}<N_{B-w}^{\prime}>\right)\right.} \\
&\left.+\left(\omega^{2} e m l\right)\left(\left\{N_{B-w}\right\}<N_{T-\varphi}>+\left\{N_{T-\varphi}\right\}<N_{B-w}>\right)\right] d \xi
\end{aligned}
$$

The same semi-circular beam configuration as presented in Figure 4.6 was then selected to investigate the DFE application to free vibration analysis of axially loaded beams. The beam properties are $[20,23]$ :

$$
\begin{aligned}
& \boldsymbol{L}=0.82 \mathrm{~m} \\
& \boldsymbol{e}=0.0155 \mathrm{~m} \\
& \boldsymbol{m}=0.835 \mathrm{~kg} / \mathrm{m} \\
& \boldsymbol{E} \boldsymbol{I}=6380.14 \mathrm{~N} \cdot \mathrm{m}^{2} \\
& \boldsymbol{G J}=43.46 \mathrm{~N} \cdot \mathrm{m}^{2} \\
& \boldsymbol{m} \cdot \boldsymbol{k}_{\boldsymbol{m}}{ }^{2}=0.000501 \mathrm{~kg} \cdot \mathrm{m}
\end{aligned}
$$

For two cases of $T=0$ and $T=-1790(N)$, the natural frequencies of beam were calculated, and the comparisons between DFE, FEM (Chapter 4) and reference values [20] are presented in Tables 5.5 and 5.6. 
Table 5.5: The bending-torsion natural frequencies

of the cantilever beam (no axial load $(T=0)$ )

\begin{tabular}{cccccc}
\hline $\begin{array}{c}\text { Natural } \\
\text { Frequency } \\
(\text { rad/s) }\end{array}$ & $\begin{array}{c}\text { Reference } \\
\text { Frequency } \\
{[20]}\end{array}$ & $\begin{array}{c}\text { DFE with } \\
6 \text { elements }\end{array}$ & $\begin{array}{c}\text { Error of DFE } \\
(\%)\end{array}$ & $\begin{array}{c}\text { FEM with } \\
\text { 20 elements }\end{array}$ & $\begin{array}{c}\text { Error of FEM } \\
(\%)\end{array}$ \\
\hline$\omega_{1}$ & 62.60 & 62.60 & 0.00 & 62.61 & 0.02 \\
$\omega_{2}$ & 130.18 & 130.75 & 0.44 & 130.21 & 0.02 \\
$\omega_{3}$ & 261.15 & 261.56 & 0.16 & 261.66 & 0.20 \\
$\omega_{4}$ & 421.36 & 423.25 & 0.45 & 423.59 & 0.53 \\
$\omega_{5}$ & 612.09 & 616.03 & 0.64 & 618.52 & 1.05 \\
\hline
\end{tabular}

Table 5.6: The bending-torsion natural frequencies of the axially loaded cantilever beam $(T=-1790 \mathrm{~N})$

\begin{tabular}{cccccc}
\hline $\begin{array}{c}\text { Natural } \\
\text { Frequency } \\
(\mathrm{rad} / \mathrm{s})\end{array}$ & $\begin{array}{c}\text { Reference } \\
\text { Frequency } \\
{[20]}\end{array}$ & $\begin{array}{c}\text { DFE with } \\
6 \text { elements }\end{array}$ & $\begin{array}{c}\text { Error of DFE } \\
(\%)\end{array}$ & $\begin{array}{c}\text { FEM with } \\
\text { 20 elements }\end{array}$ & $\begin{array}{c}\text { Error of FEM } \\
(\%)\end{array}$ \\
\hline$\omega_{1}$ & 60.23 & 60.25 & 0.04 & 60.24 & 0.02 \\
$\omega_{2}$ & 128.42 & 128.74 & 0.25 & 128.45 & 0.02 \\
$\omega_{3}$ & 257.96 & 258.48 & 0.20 & 258.46 & 0.19 \\
$\omega_{4}$ & 415.54 & 416.79 & 0.30 & 417.74 & 0.53 \\
$\omega_{5}$ & 604.60 & 607.87 & 0.54 & 611.12 & 1.08 \\
\hline
\end{tabular}

The DFE convergence results for the beam subjected to a compressive axial load of $1790(N)$ are shown in Figure 5.7. One can see that for the first four frequencies the error for a 4 -element model is less than $0.8 \%$ (the error for the first frequency obtained by this model is less than $0.1 \%$ ). Therefore, using 4 to 6 elements, one could accurately evaluate the beam's first few frequencies. 


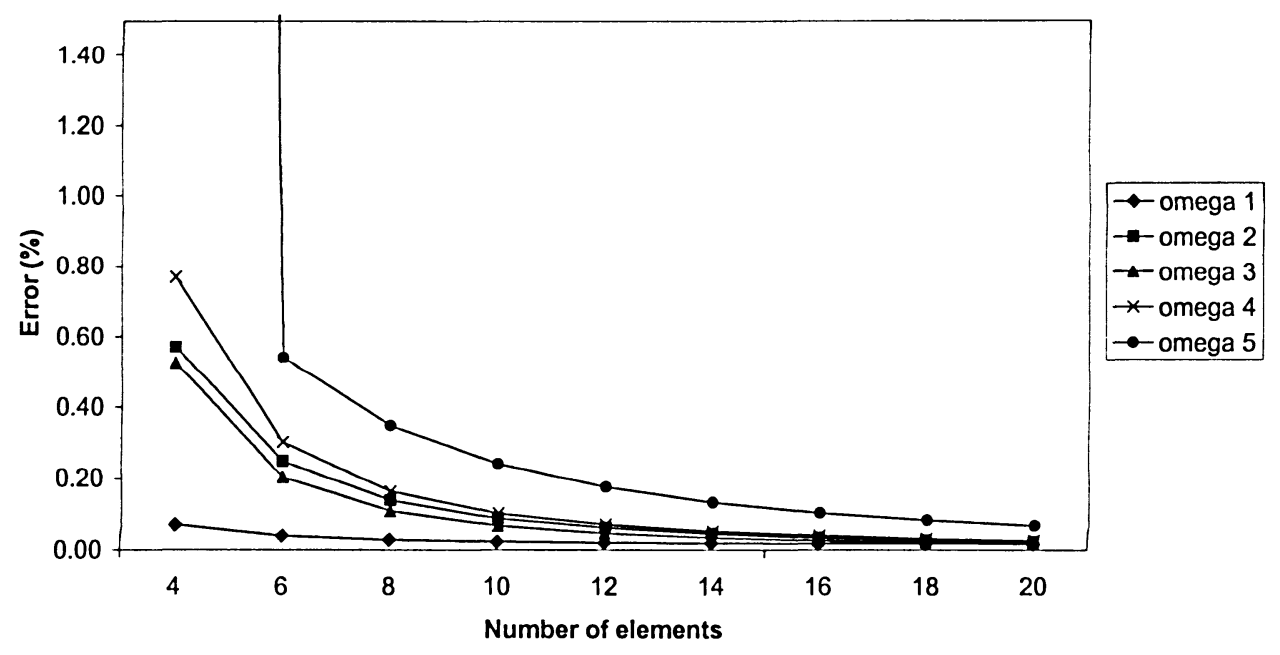

Figure 5.7: The DFE convergence results for axially loaded cantilever coupled beam $(T=-1790 \mathrm{~N})$.

\subsubsection{Coupled Bending-Bending Vibrations}

The DFE formulation was then applied to the free vibration analysis of coupled bending-bending rotating beams. The element stiffness matrix, equation (5.35), reduces to:

$$
[K(\omega)]^{e}=[K(\omega)]_{\text {Uncoupled }}^{e}+[K(\omega)]_{\text {Coupled }}^{e}
$$

where

$$
\begin{aligned}
& {[K(\omega)]_{\text {Uncoupled }}^{e}=} \\
& {[\overbrace{c_{w}\left\{N_{B-w}^{m}\right\}-c_{T}\left\{N_{B-w}^{\prime}\right\} ;-c_{w}\left\{N_{B-w}^{n}\right\} ; c_{v}\left\{N_{B-v}^{m}\right\}-c_{T}\left\{N_{B-v}^{\prime}\right\} ;-c_{v}\left\{N_{B-v}^{n}\right\}}^{\xi=0} ;} \\
& \overbrace{c_{T}\left\{N_{B-w}^{\prime}\right\}-c_{w}\left\{N_{B-w}^{m}\right\} ; c_{w}\left\{N_{B-w}^{n}\right\} ; c_{T}\left\{N_{B-v}^{\prime}\right\}-c_{v}\left\{N_{B-v}^{m}\right\} ; c_{v}\left\{N_{B-v}^{n}\right]}^{\xi=1} \\
& +\int_{0}^{+1}\left[\left(-\Omega^{2} m l\right)\left\{N_{B-v}\right\}<N_{B-v}>\right] d \xi
\end{aligned}
$$


and

$$
[K(\omega)]_{\text {Coupled }}^{e}=\int_{0}^{+1}\left[\left(\frac{E I_{z y}}{l^{3}}\right)\left(\left\{N_{B-w}^{n}\right\}<N_{B-v}^{n}>+\left\{N_{B-v}^{n}\right\}<N_{B-w}^{n}>\right)\right] d \xi
$$

where the vectors of nodal variables $\{\boldsymbol{u}\}$ and shape functions $\left\langle N_{\boldsymbol{B - \boldsymbol { w }}}>\right.$ and $\left\langle N_{\boldsymbol{B - v} \boldsymbol{v}}\right\rangle$ are written as follow:

$$
\begin{gathered}
\{u\}=<\begin{array}{lllllllll}
w_{1} & w_{1}^{\prime} & v_{1} & v_{1}^{\prime} & w_{2} & w_{2}^{\prime} & v_{2} & v_{2}^{\prime}>^{T} \\
<N_{B-w}> & =<N_{B 1} \quad N_{B 2} & 0 & 0 & N_{B 3} & N_{B 4} & 0 & 0
\end{array}> \\
<N_{B-v}>=<\begin{array}{llllllllll}
0 & 0 & N_{B 1} & N_{B 2} & 0 & 0 & N_{B 3} & N_{B 4}>
\end{array}
\end{gathered}
$$

In next sections coupled bending-bending beams with constant axial load and centrifugal forces will be investigated to validate the DFE for this category of structures.

\subsubsection{Axially Loaded Beam:}

The uncoupled bending-bending vibrations of a cantilever beam with symmetric cross section under constant axial load was first studied. The beam geometric and mechanical properties are as follow:

$$
\begin{aligned}
& L=7.0 \mathrm{~m} \\
& \boldsymbol{m}=4.5 \mathrm{~kg} / \mathrm{m} \\
& T=1000 \mathrm{~N} \\
& E I_{\xi}=3000 \mathrm{~N} . \mathrm{m}^{2} \\
& E I_{\eta}=3000 \mathrm{~N} . \mathrm{m}^{2}
\end{aligned}
$$


Since the flexural rigidities in both directions are the same, it is expected to obtain repeated frequencies for in-plane and out-of-plane free vibrations. Table 5.7 shows the first six natural frequencies of the Beam. As already mentioned, the DFE for uncoupled beam is an exact method and the error for DFE with one element is zero. One can see that the FEM errors for the first few natural frequencies are also within the acceptable range and the FEM follows the general pattern such that the error increases for higher frequencies.

Table 5.7: The uncoupled in- and out-of-plane bending natural frequencies of the cantilever beam

\begin{tabular}{cccccc}
\hline $\begin{array}{c}\text { Natural } \\
\text { Frequency } \\
\text { (rad/s) }\end{array}$ & $\begin{array}{c}\text { Exact } \\
\text { value }\end{array}$ & $\begin{array}{c}\text { DFE with } \\
\text { 1 element }\end{array}$ & $\begin{array}{c}\text { Error of DFE } \\
(\%)\end{array}$ & $\begin{array}{c}\text { FEM with } \\
\text { 10 elements }\end{array}$ & $\begin{array}{c}\text { Error of FEM } \\
(\%)\end{array}$ \\
\hline$\omega_{1}$ & 4.4821 & 4.4821 & 0.000 & 4.4822 & 0.001 \\
$\omega_{2}$ & 4.4821 & 4.4821 & 0.000 & 4.4822 & 0.001 \\
$\omega_{3}$ & 16.5835 & 16.5835 & 0.000 & 16.5843 & 0.005 \\
$\omega_{4}$ & 16.5835 & 16.5835 & 0.000 & 16.5843 & 0.005 \\
$\omega_{5}$ & 37.4918 & 37.4918 & 0.000 & 37.5011 & 0.025 \\
$\omega_{6}$ & 37.4918 & 37.4918 & 0.000 & 37.5011 & 0.025 \\
\hline
\end{tabular}

If a pre-twist angle $\theta$ is added to the system (i.e., rotation of principle axes around longitudinal axes $x$ ) then the beam will experience the coupled bending-bending vibrations. The pre-twist angle causes geometric coupling between two bending displacements by producing the coupling term $\boldsymbol{E} \boldsymbol{I}_{\boldsymbol{z} \boldsymbol{y}}$. The frequencies for the per-twisted beam, in this case, are the same as the uncoupled beam due to the fact that rotation of the coordinate system doesn't change the nature of the system. The natural frequencies in coupled case also confirm the DFE's higher convergence rates comparing to FEM, where the DFE errors with only one element for the first six frequencies for this example are zero.

Even though constant force can be used to estimate the effect of centrifugal force, as it was used for coupled bending-torsion beam, but there is a principal difference between bending-torsion coupled vibrations and bending-bending coupled vibrations. In 
the latter case, the centripetal term decreases the rigidity of the beam in $y$-direction, which cannot be taken into account when the axial force is simulated by constant force. In Figure 5.8 the effect of a constant axial force on the first six natural frequencies of the beam are shown.

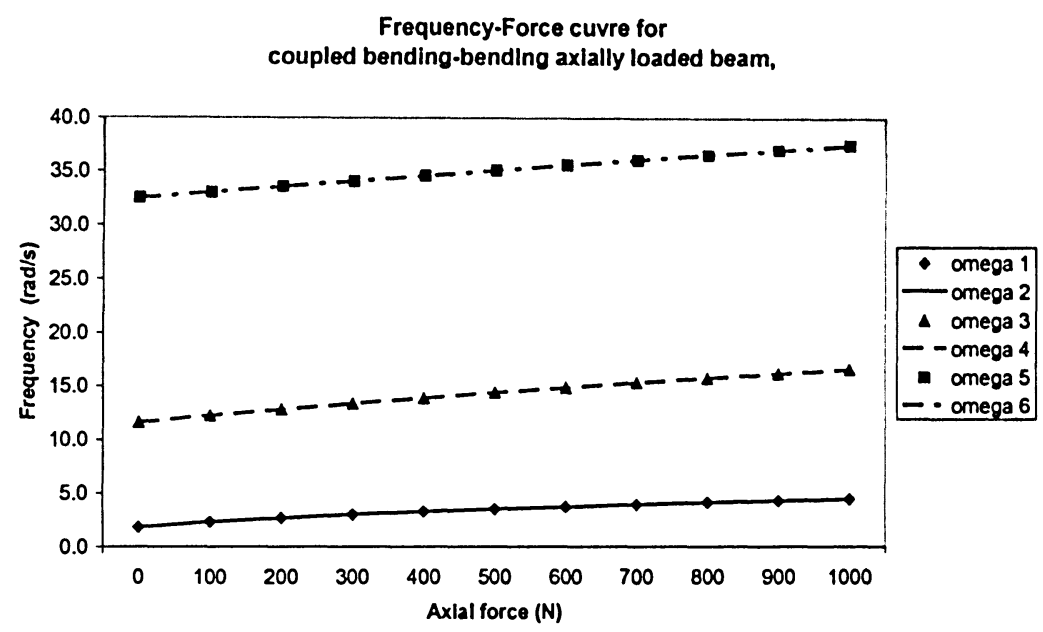

Figure 5.8: The effect of axial force on natural frequencies of clamped-free beam bending-bending vibrations.

One can see (Figure 5.8) that by constant axial force the flap frequencies and lag frequencies increase with the same rate (for this special case they are exactly the same). It will be shown later that when the effect of centripetal force is taken into account, the increment rate of flap and lag frequencies are not the same.

\subsubsection{Coupled Bending-Bending Rotating Beams:}

For rotating beams, the equations (5.55) to (5.57) are used to build the system's frequency dependent (nonlinear) stiffness matrix. In this case the centrifugal and centripetal terms both contribute in stiffness matrix, when the former increases and the latter decreases the stiffness of the system. 
Using the same beam configuration as discussed in the previous section, and incorporating a constant pre-twist angle $\theta=30^{\circ}$, the beam natural frequencies for rotating speed $\mathbf{\Omega}=5(\mathrm{rad} / \mathrm{s})$ were calculated and shown in Table 5.8:

Table 5.8: The coupled bending-bending natural frequencies of a rotating cantilever beam, $\Omega=5(\mathrm{rad} / \mathrm{s})$

\begin{tabular}{cccc}
\hline $\begin{array}{c}\text { Natural Frequency } \\
\text { (rad/s) }\end{array}$ & $\begin{array}{c}\text { Reference } \\
\text { FEM, } n=500\end{array}$ & $\begin{array}{c}\text { DFE with } \\
5 \text { element }\end{array}$ & $\begin{array}{c}\text { Error of DFE } \\
\text { (\%) }\end{array}$ \\
\hline$\omega_{1}$ & 2.619 & 2.608 & 0.422 \\
$\omega_{2}$ & 5.645 & 5.640 & 0.079 \\
$\omega_{3}$ & 16.494 & 16.479 & 0.094 \\
$\omega_{4}$ & 17.235 & 17.221 & 0.085 \\
$\omega_{5}$ & 38.409 & 38.396 & 0.035 \\
$\omega_{6}$ & 38.733 & 38.720 & 0.035 \\
\hline
\end{tabular}

Table 5.8 shows excellent convergence of DFE in comparison with FEM, where the error for $5^{\text {th }}$ and $6^{\text {th }}$ frequencies is less than $0.04 \%$. The error percentage versus number of elements for DFE method are presented in Figure 5.9:

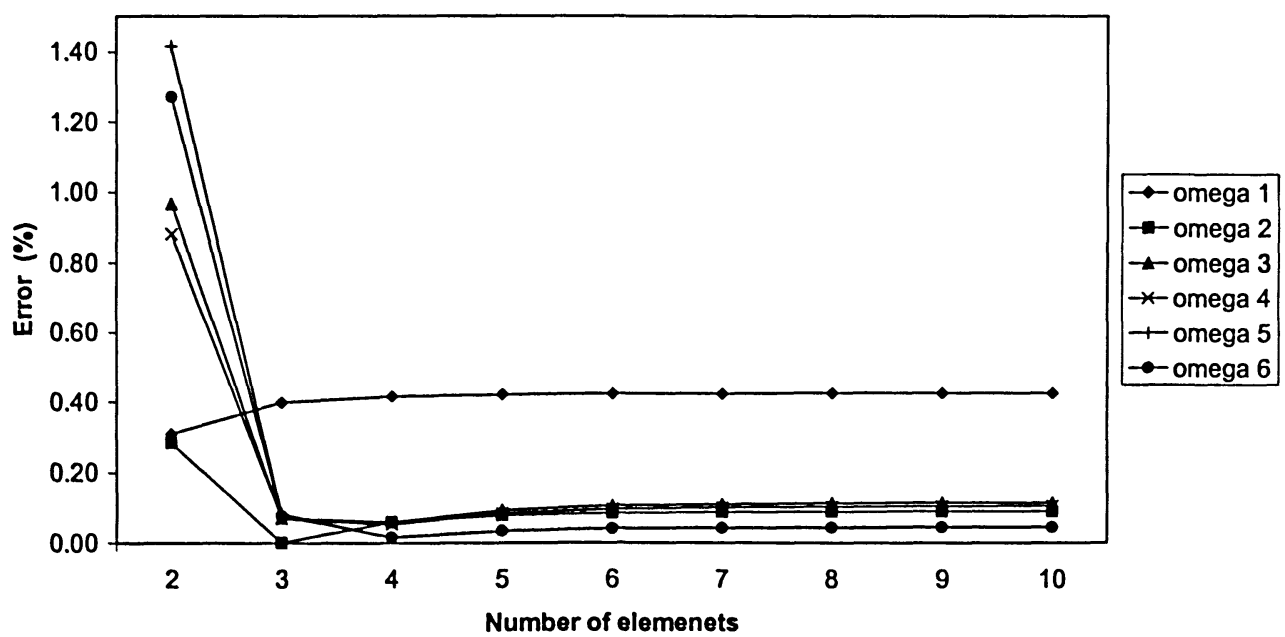


Figure 5.9: The DFE convergence results for bending-bending

vibrations of a rotating beam.

The first three flap and lag natural frequencies of the rotating beam are shown in Figure 5.10.

The effect of rotary speed on natural frequencies for coupled bending-bending rotating beam

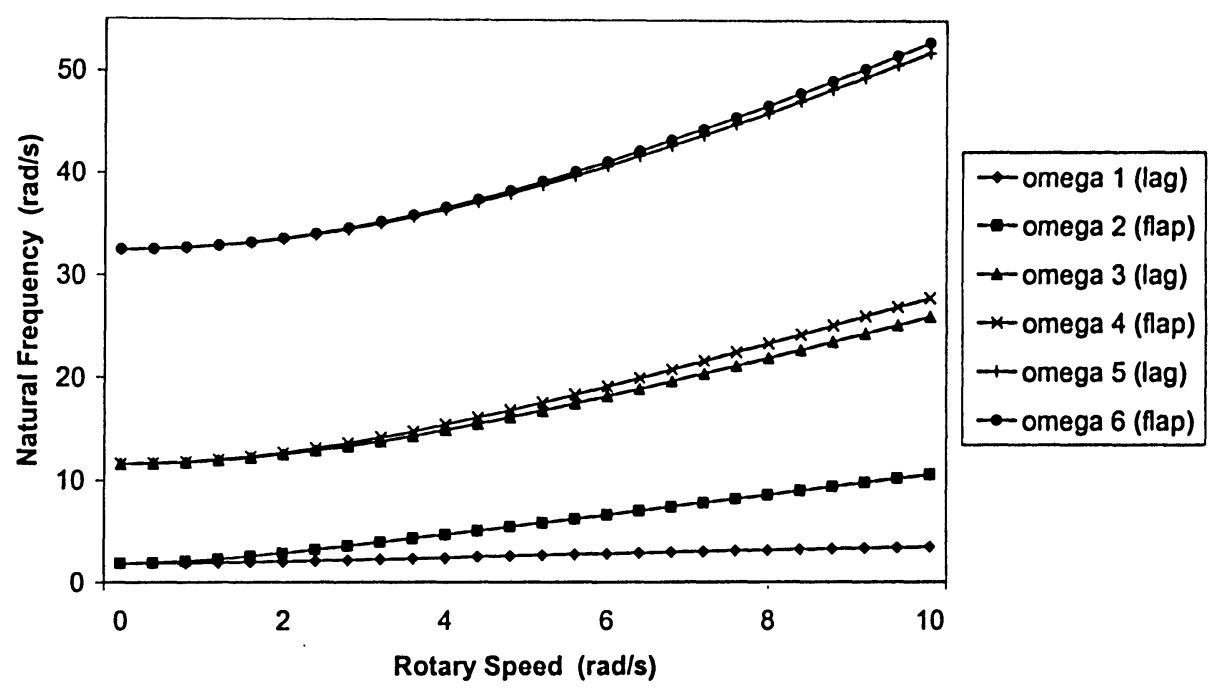

Figure 5.10: The effect of rotating speed on natural frequencies for clamped-free beam bending-bending vibrations.

Figure 5.10 shows that the growth of lag frequencies is slower than flap frequencies due to the centripetal effect on the in-plane vibrations which plays a softening role and decreases the stiffness of beam in lag direction. On the other hand, the difference between flap and lag frequencies decreases for higher modes. Also one can see that higher frequencies grow faster than lower frequencies.

In order to investigate the natural modes of coupled bending-bending rotating beam, a uniform beam with following properties is considered in which the flexural rigidities in two directions are different: 


$$
\begin{aligned}
& \boldsymbol{L}=7.0 \mathrm{~m} \\
& \boldsymbol{m}=4.5 \mathrm{~kg} / \mathrm{m} \\
& \boldsymbol{\theta}=30 \text { degree } \\
& \boldsymbol{E} \boldsymbol{I}_{\eta}=3000 \mathrm{~N} . \mathrm{m}^{2} \\
& \boldsymbol{E} \boldsymbol{I}_{\xi}=7500 \mathrm{~N} . \mathrm{m}^{2} \\
& \mathbf{\Omega}=5 \mathrm{rad} / \mathrm{s}
\end{aligned}
$$

The first five natural frequencies were calculated and compared with FEM method. Reference frequencies have been obtained from a 600-element FEM model. The results are summarized in Table 5.9.

Table 5.9: The coupled bending-bending natural frequencies

$$
\text { of the rotating beam }
$$

\begin{tabular}{cccc}
\hline $\begin{array}{c}\text { Natural Frequency } \\
\text { (rad/s) }\end{array}$ & $\begin{array}{c}\text { Reference } \\
\text { frequency } \\
\text { FEM, } n=600\end{array}$ & $\begin{array}{c}\text { DFE with } \\
\text { 3 element }\end{array}$ & $\begin{array}{c}\text { Error of DFE } \\
\text { (\%) }\end{array}$ \\
\hline$\omega_{1}$ & 3.307 & 3.301 & 0.202 \\
$\omega_{2}$ & 5.795 & 5.796 & 0.013 \\
$\omega_{3}$ & 17.032 & 17.038 & 0.032 \\
$\omega_{4}$ & 21.940 & 21.952 & 0.058 \\
$\omega_{5}$ & 38.648 & 38.748 & 0.257 \\
\hline
\end{tabular}

Table 5.9 shows the excellent convergence rate for the DFE, where using only three elements results in an error less than $0.3 \%$. The mode shapes corresponding to the first eight frequencies are shown in Figure 5.11. 

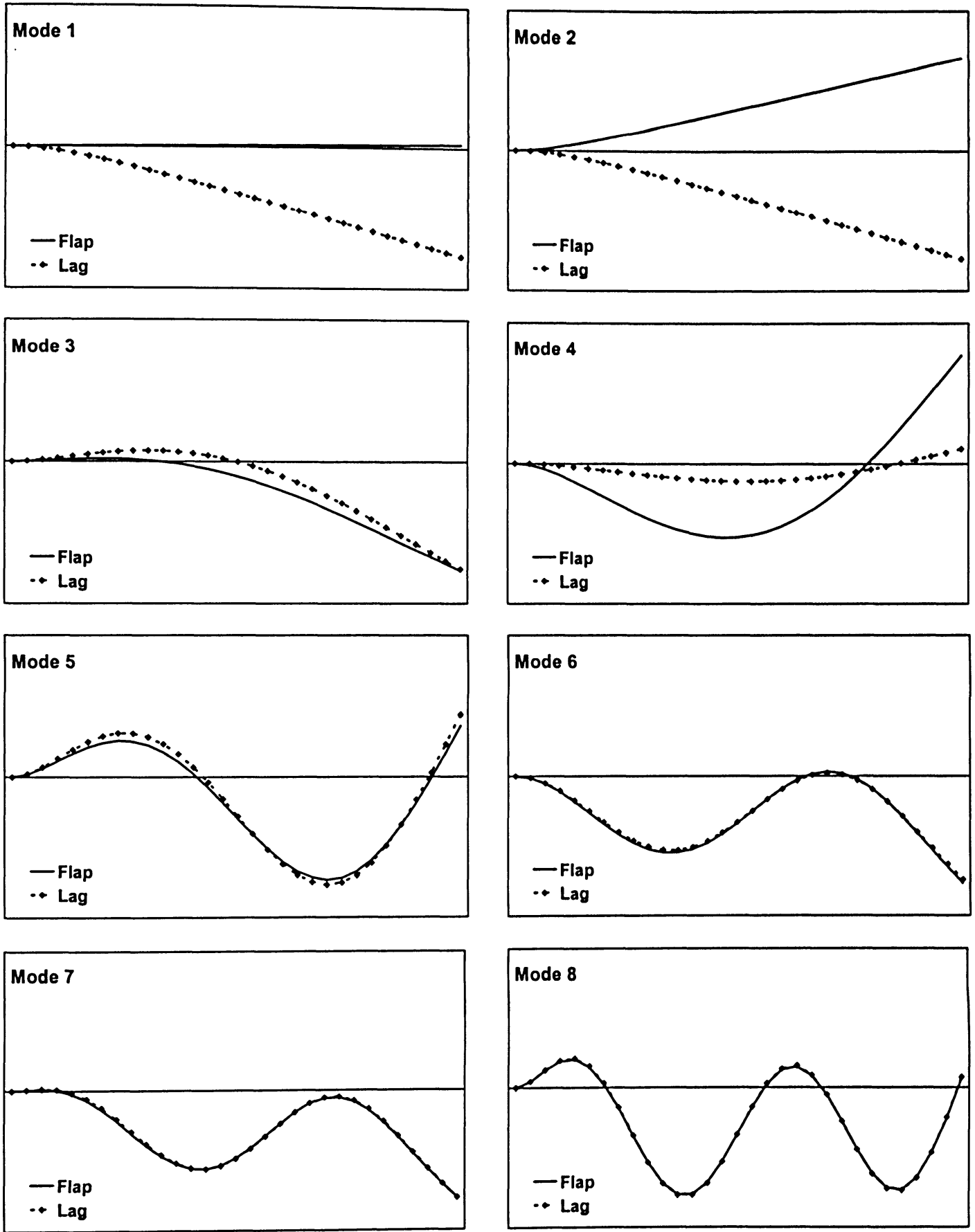

Figure 5.11: The first eighth mode shapes of bending-bending vibrations of a clamped-free rotating beam.

The mode shapes (Figure 5.11) exhibit the expected behaviour, where for higher modes the number of nodes (zero displacement point on beam) increases. 


\subsubsection{Rotating Blade with Variable Cross Section:}

In order to demonstrate the applicability of the DFE approach to complex problems, the rotating coupled bending-bending blade with variable cross section, introduced in section 4.5.3, was then studied. The first three natural frequencies at different rotating speed $\mathbf{\Omega}$ were calculated and are shown in Table 5.10 .

Table 5.10: The first three natural frequencies $(\mathrm{Hz})$ of vibrations of propeller blade WADC S-5 (DFE method)

\begin{tabular}{|c|c|c|c|c|c|c|}
\hline $\begin{array}{c}\text { Blade rotary } \\
\text { speed } \\
\text { (rpm) }\end{array}$ & $\begin{array}{c}\text { Mode } \\
\text { number }\end{array}$ & $\begin{array}{c}D F E \\
n=9\end{array}$ & $\begin{array}{l}F E M \\
n=9\end{array}$ & $\begin{array}{c}\text { Result of } \\
\text { Reference } \\
\text { [2] }\end{array}$ & $\begin{array}{c}\text { Result of } \\
\text { Reference } \\
\text { [5] }\end{array}$ & $\begin{array}{c}\text { Experiment } \\
\text { [27] }\end{array}$ \\
\hline & 1 & 40.82 & 40.32 & 40.96 & 39.89 & 40.08 \\
\hline \multirow[t]{3}{*}{1567} & 2 & 109.54 & 108.49 & 109.22 & 107.40 & \\
\hline & 3 & 280.30 & 279.27 & 279.79 & 276.32 & \\
\hline & 1 & 41.22 & 40.70 & 41.35 & 40.26 & \\
\hline \multirow[t]{3}{*}{1589} & 2 & 110.10 & 109.01 & 109.77 & 107.93 & 107.53 \\
\hline & 3 & 280.98 & 279.92 & 280.47 & 276.97 & \\
\hline & 1 & 59.74 & 58.99 & 60.07 & 58.05 & 58.73 \\
\hline \multirow[t]{3}{*}{2609} & 2 & 139.47 & 137.18 & 139.52 & 135.99 & \\
\hline & 3 & 319.42 & 316.82 & 309.40 & 313.98 & \\
\hline & 1 & 59.84 & 59.08 & 60.16 & 58.14 & \\
\hline \multirow[t]{3}{*}{2614} & 2 & 139.63 & 137.33 & 139.68 & 136.14 & 137.02 \\
\hline & 3 & 319.63 & 317.03 & 319.62 & 314.19 & \\
\hline & 1 & 77.78 & 76.87 & 78.34 & 75.30 & 76.52 \\
\hline \multirow[t]{3}{*}{3583} & 2 & 171.49 & 168.08 & & 166.25 & 170.60 \\
\hline & 3 & 365.76 & 361.38 & & 357.70 & \\
\hline & 1 & 94.53 & 93.48 & & 91.42 & 93.07 \\
\hline \multirow[t]{3}{*}{4486} & 2 & 202.27 & 197.91 & & & \\
\hline & 3 & 413.43 & 407.24 & & & \\
\hline & 1 & 95.48 & 94.42 & & & \\
\hline \multirow[t]{3}{*}{4537} & 2 & 204.02 & 199.61 & & 196.41 & 202.53 \\
\hline & 3 & 416.20 & 409.91 & & & \\
\hline & 1 & 120.41 & 119.17 & & 116.07 & 117.50 \\
\hline \multirow[t]{2}{*}{5884} & 2 & 249.67 & 244.05 & & & \\
\hline & 3 & 490.10 & 481.05 & & & \\
\hline
\end{tabular}


The DFE results are in good agreement with those of Transfer Matrix Method (TTM) [2], where the error for angular speed of 1567 (rpm) and 1589 (rpm) is less than $0.2 \%$. This example shows the applicability of DFE method for complex geometries where the exact method cannot be used.

Reviewing all examples of this section, one can see that the DFE method can be advantageously used to calculate the natural frequencies and mode shapes of vibrations of bending-bending coupled beam with different levels of complexity.

\subsubsection{Coupled Bending-Bending-Torsion Vibrations}

Bending-bending-torsion coupled beams can be used in simulation of the turbine or propeller blades or aircraft wings. For short beams, the shear deformations and rotary inertia must be considered to provide a more accurate model, and in some cases it is necessary to consider the thermal effect where the temperature is considerably higher than ambient temperature [19]. For propeller blades and aircraft wings the geometry of beam allows using Euler-Bernoulli assumptions and the results are accurate enough for vibration analysis of the first few modes of vibrations.

The coupling between differential equations governing the flap-wise bending, cord-wise bending and torsion occurs because of the beam's cross-sectional geometry, due to the distance between mass and elastic (shear) centers, $e$, and asymmetry of the cross section.

In this section, by a progressive approach, the free vibrations of triply coupled bending-bending-torsion beams for non-rotating and rotating cases are discussed.

\subsubsection{Uncoupled Beam Vibrations}

As already stated, for uncoupled equations of motion, the DFE method results in the exact solutions. The uncoupled equations of motion for lag (in-plane) bending, flap (out-of-plane) bending and torsion vibrations of a beam can be written as: 


$$
\begin{gathered}
\left(E I_{y} w^{\prime \prime}\right)^{n}-\omega^{2} m w=0 \\
\left(E I_{z} v^{\prime \prime}\right)^{\prime \prime}-\omega^{2} m v=0 \\
-\left(G J \phi^{\prime}\right)^{\prime}-\omega^{2} m \kappa_{m}^{2} \phi=0
\end{gathered}
$$

By implementing the DFE method to the equations (5.61), (5.62) and (5.63), the exact uncoupled in- and out-of-plane bending and torsion natural frequencies as discussed in Chapter 4, were obtained. These results are also in agreement with FEM (see section 4.5.4.1 and Table 4.10).

\subsubsection{Vibrations of Non-rotating Coupled Beam}

For a non-rotating beam, the eccentricity $e$ and the asymmetry produce the coupling terms, and the element stiffness matrix expressions (5.35) to (5.37) reduces to the following form:

$$
[K(\omega)]^{e}=[K(\omega)]_{\text {Uncoupled }}^{e}+[K(\omega)]_{\text {Coupled }}^{e}
$$

Where

$$
\begin{aligned}
& {[K(\omega)]_{\text {Uncoupled }}^{e}=} \\
& {[\overbrace{c_{w}\left\{N_{B-w}^{m}\right\} ;-c_{w}\left\{N_{B-w}^{n}\right\} ; c_{\nu}\left\{N_{B-\nu}^{m}\right\} ;-c_{v}\left\{N_{B-v}^{n}\right\} ;-c_{\varphi}\left\{N_{T-\phi}^{\prime}\right\}}^{\xi=0} ;} \\
& \overbrace{-c_{w}\left\{N_{B-w}^{m}\right\} ; c_{w}\left\{N_{B-w}^{n}\right\} ;-c_{v}\left\{N_{B-v}^{m}\right\} ; c_{v}\left\{N_{B-v}^{n}\right\} ; c_{\varphi}\left\{N_{T-\varphi}^{\prime}\right\}}^{\xi=1}]
\end{aligned}
$$

and 


$$
\begin{aligned}
{[K(\omega)]_{\text {Coupled }}^{e} } & =\int_{0}^{+1}\left[\left(\frac{E I_{z y}}{l^{3}}\right)\left(\left\{N_{B-w}^{\prime \prime}\right\}<N_{B-\nu}^{\prime \prime}>+\left\{N_{B-v}^{*}\right\}<N_{B-w}^{\prime \prime}>\right)\right. \\
& -\left(\omega^{2} e m l \cos \theta\right)\left(\left\{N_{B-w}\right\}<N_{T-\phi}>+\left\{N_{T-\phi}\right\}<N_{B-\omega}>\right) \\
& \left.+\left(\omega^{2} e m l \sin \theta\right)\left(\left\{N_{B-v}\right\}<N_{T-\phi}>+\left\{N_{T-\phi}\right\}<N_{B-v}>\right)\right] d \xi
\end{aligned}
$$

In order to investigate the applicability of DFE to the triply coupled vibrations of a non-rotating pre-twisted beam, the beam configuration studied in section 4.5.4.2 reinvestigated $[28,29]$. The geometric and material properties of the beam are:

$$
\begin{aligned}
& \boldsymbol{L}=40.0 \mathrm{in} \\
& \boldsymbol{e}=1.414 \mathrm{in} \\
& \theta=45 \mathrm{deg} \text { (constant along the beam length) } \\
& \boldsymbol{m}=0.0015 \mathrm{slug} / \mathrm{in} \\
& \boldsymbol{k}_{\boldsymbol{m} I}=\boldsymbol{k}_{\boldsymbol{m} 2}=1.0 \mathrm{in} \\
& \boldsymbol{E} I_{\eta}=25000 \mathrm{lb} . \mathrm{in}^{2} \\
& \boldsymbol{E} \boldsymbol{I}_{\xi}=75000{\mathrm{lb} . \mathrm{in}^{2}}^{2} \\
& \boldsymbol{G J}=9000{\mathrm{lb} . \mathrm{in}^{2}}^{2}
\end{aligned}
$$

where the pre-twist angle, $\theta$, assumed to be constant along the beam length. The beam natural frequencies obtained from the proposed DFE method are shown in Table 5.11. The reference values for beam natural frequencies, in this case, are those obtained from "Transfer Matrix Method (TMM)" published by Murthy [2]. The comparison between DFE and FEM results confirms the superiority of the proposed DFE method (Table 5.11). The convergence results for the FEM and DFE methods for the first, third and fifth frequencies are also compared in Figure 5.12. 
Table 5.11: The triply coupled bending-bending-torsion natural frequencies of the pre-twisted clamped-free beam

\begin{tabular}{cccccc}
\hline $\begin{array}{c}\text { Natural } \\
\text { Frequency } \\
(\text { rad/s) }\end{array}$ & TMM [2] & $\begin{array}{c}\text { DFE } \\
n=10\end{array}$ & $\begin{array}{c}\text { Errorfor } \\
\text { DFE } \\
(\%)\end{array}$ & $\begin{array}{c}\text { FEM } \\
n=10\end{array}$ & $\begin{array}{c}\text { Error for } \\
\text { FEM } \\
(\%)\end{array}$ \\
\hline$\omega_{1}$ & 30.8295 & 30.8300 & 0.001 & 30.8300 & 0.002 \\
$\omega_{2}$ & 53.8277 & 53.8278 & 0.000 & 53.8278 & 0.000 \\
$\omega_{3}$ & 184.6175 & 184.7138 & 0.052 & 184.7376 & 0.065 \\
$\omega_{4}$ & 337.3333 & 337.3351 & 0.001 & 337.3440 & 0.003 \\
$\omega_{5}$ & 484.3373 & 485.7737 & 0.297 & 486.4762 & 0.442 \\
\hline
\end{tabular}



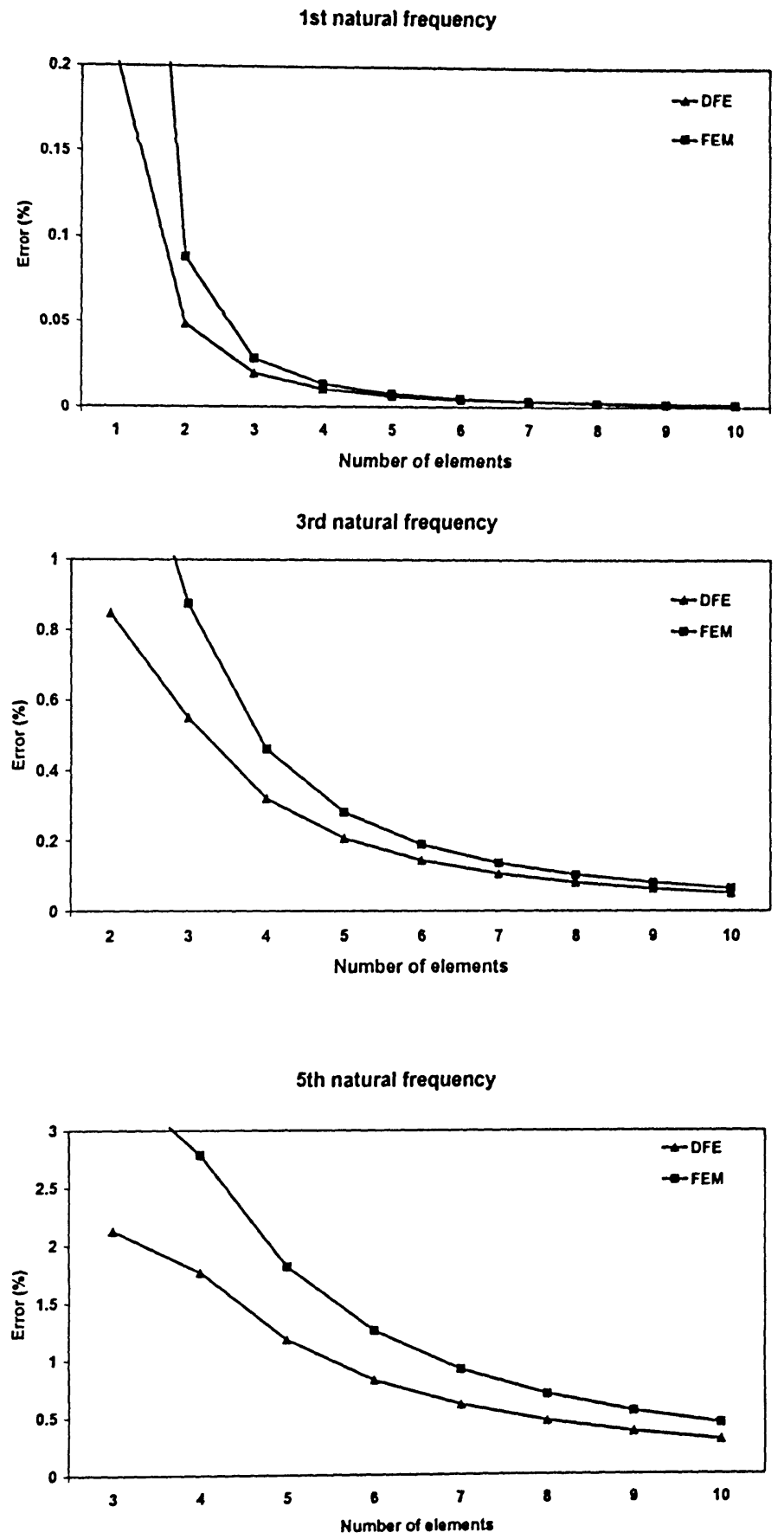

Figure 5.12: The comparison between the DFE and FEM convergence for non-rotating triply coupled beam. 
Investigation in mode shapes reveals that the modes obtained by DFE and FEM are identical. The first five modes corresponding to the first five natural frequencies are shown in Figure 5.13 (Note: the first, second and fourth torsion modes have been magnified by $10,10^{8}$ and $10^{4}$ respectively to be visible).
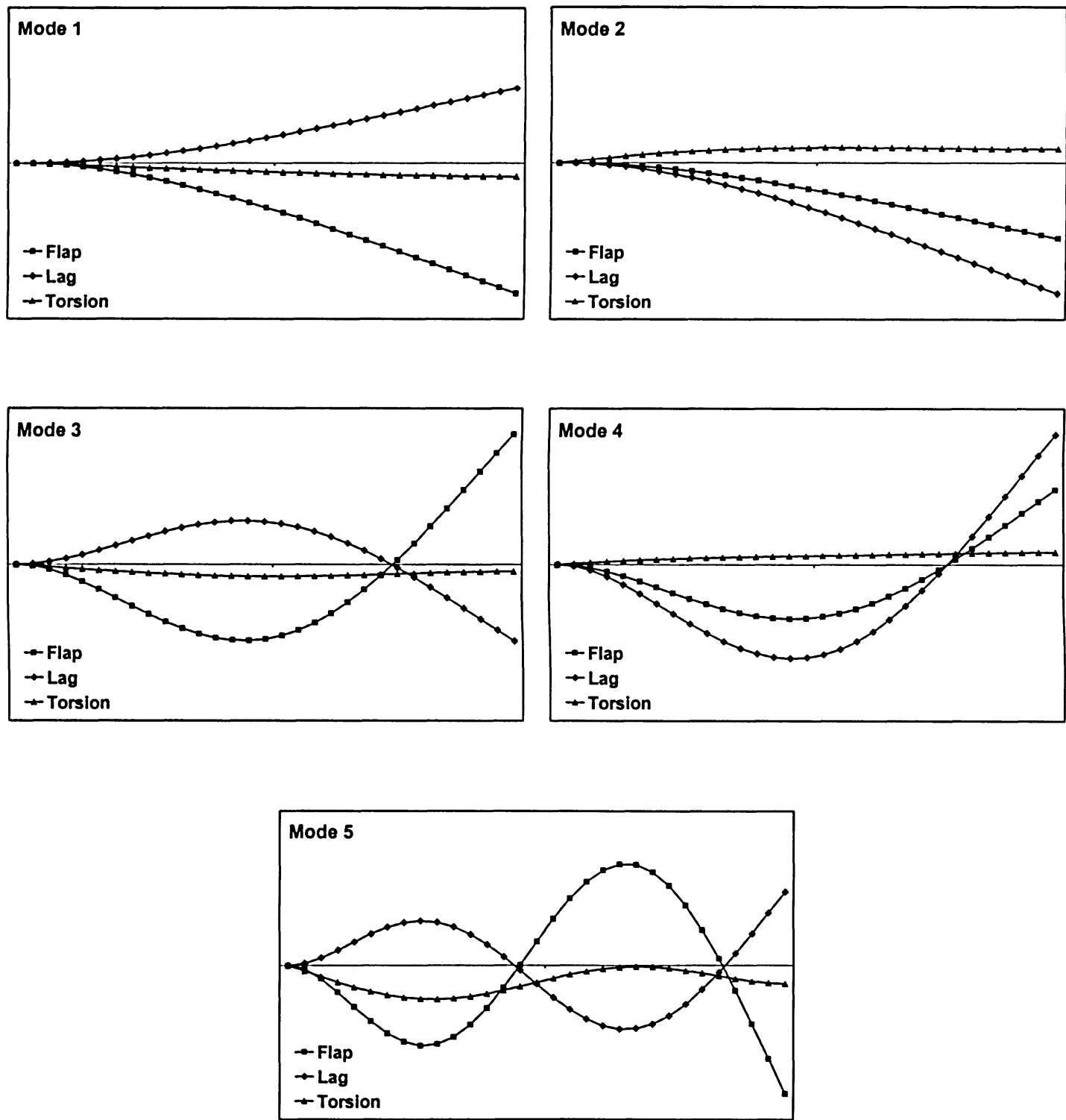

Figure 5.13: The first three modes of vibrations of non-rotating triply coupled beam. 


\subsubsection{Vibrations of Rotating Triply Coupled Beam}

For a rotating pre-twisted beam, the governing equations of motion are in the form of (2.1), (2.2) and (2.3). These equations can be used to model the low frequency vibrations of high aspect ratio compressor, turbine, propeller and helicopter blades. The element dynamic stiffness matrix, consisting of coupled and uncoupled stiffness matrices, can be written as introduced in equations (5.35) to (5.37). By including the deviator matrix, the uncoupled stiffness matrix then changes to equation (5.45).

Here, the free vibration of a triply coupled pre-twisted cantilever helicopter blade is studied. The following geometric, mechanical and material properties, as reported in the literature $[4,6,16]$ (see also section 4.5.4.3) were used:

$$
\begin{aligned}
& L=208 \text { in } \\
& \boldsymbol{e}=-0.6 \text { in } \\
& e_{1}=52 \text { in } \\
& \theta=15.026 \mathrm{deg} \text { (constant along the blade length) } \\
& \boldsymbol{\xi}=0.0015 \mathrm{lb} . \mathrm{sec}^{2} / \mathrm{in}^{2} \\
& \text { m. } \boldsymbol{k}_{\boldsymbol{m} I}{ }^{2}=0.89545 \times 10^{-3}{\mathrm{lb} . \mathrm{sec}^{2}}^{2} \\
& m . \boldsymbol{k}_{\boldsymbol{m} 2}{ }^{2}=0.04 \text { lb.sec }{ }^{2} \\
& E I_{\eta}=0.2977 \times 10^{8} \quad l b . i^{2} \\
& E \boldsymbol{I}_{\xi}=10 \times 10^{8} \mathrm{lb} \cdot \mathrm{in}^{2} \\
& \boldsymbol{G J}=0.2 \times 10^{8} \mathrm{lb} \cdot \mathrm{in}^{2} \\
& \mathbf{\Omega}=360 \mathrm{rpm}
\end{aligned}
$$

Based on the DFE method, the natural frequencies of the cantilever blade were then calculated and compared with those obtained from the FEM (Table 5.11). Since the exact results are not available, an FEM model based on 700-element mesh was used to evaluate the reference values. As it can be seen from Table 5.11, a coarse mesh of only 
seven DFE elements, leads to an infinitesimal average error of less than $0.02 \%$ when the first 5 natural frequencies are to be evaluated. The DFE convergence results compared with an FEM model (700-element mesh) are shown in Figure 5.14.

Table 5.12: The triply coupled bending-bending-torsion natural frequencies of the pre-twisted rotating beam, $\Omega=360(\mathrm{rpm})$

\begin{tabular}{cccc}
\hline $\begin{array}{c}\text { Natural Frequency } \\
\text { (rad/s) }\end{array}$ & $\begin{array}{c}\text { Reference } \\
\text { FEM } \\
n=700\end{array}$ & $\begin{array}{c}\text { DFE with } \\
7 \text { element }\end{array}$ & $\begin{array}{c}\text { Error of DFE } \\
\text { (\%) }\end{array}$ \\
\hline$\omega_{1}$ & 46.125 & 46.096 & 0.013 \\
$\omega_{2}$ & 73.253 & 73.225 & 0.001 \\
$\omega_{3}$ & 130.864 & 130.757 & 0.023 \\
$\omega_{4}$ & 171.963 & 171.965 & 0.000 \\
$\omega_{5}$ & 271.435 & 271.452 & 0.060 \\
\hline
\end{tabular}

Natural frequemcies of DFE analysis of a triply coupled rotating beam, Omega $=360 \mathrm{rpm}$

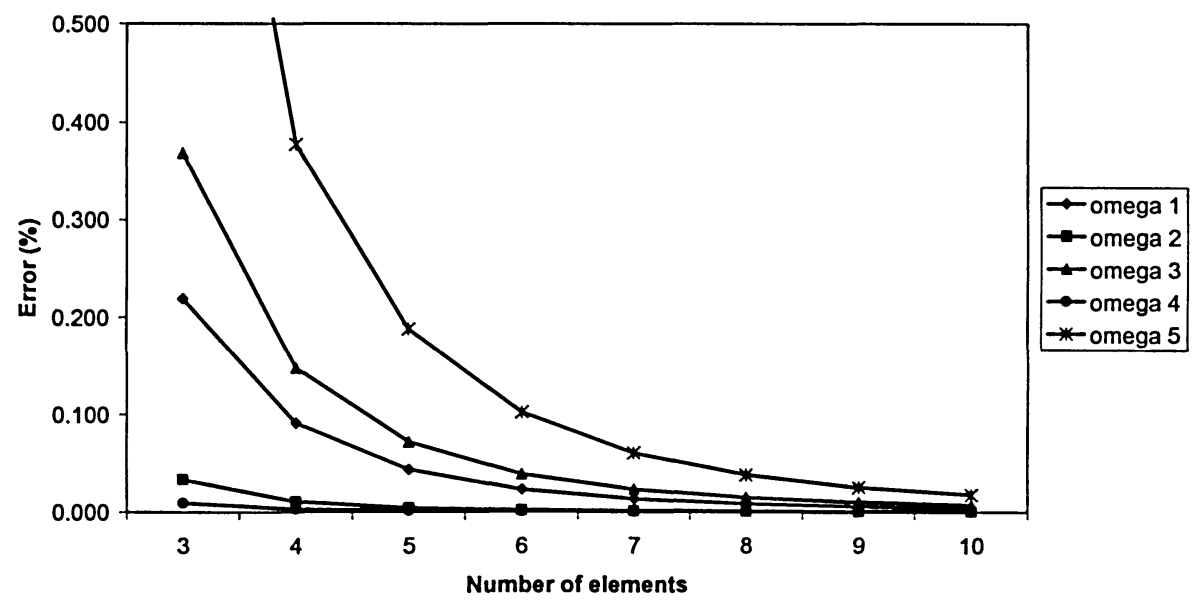

Figure 5.14: The DFE convergence results for rotating triply coupled beam.

The mode shapes of the triply coupled rotating beam were also investigated (see Figure 5.15). The results confirm the expected modal behaviour for given configuration 
(the first, second, third and fifth torsion modes have been magnified by $10^{3}, 10^{5}, 10$ and $10^{2}$ respectively to be visible).
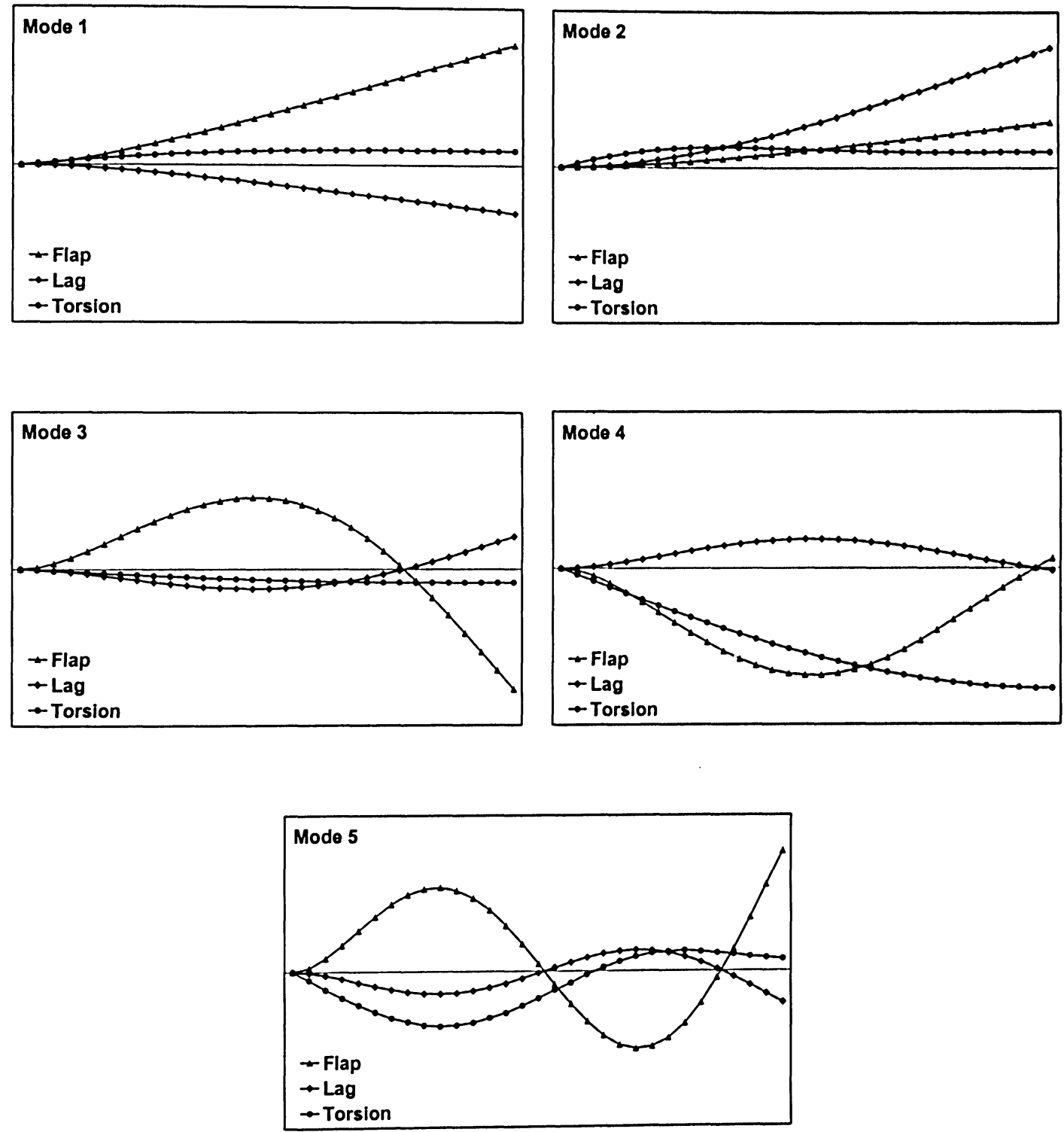

Figure 5.15: The modes of vibrations for a triply coupled rotating beam.

Furthermore, in order to validate the proposed method and to verify the applicability of DFE method to more complicated cases, a uniform linearly twisted blade 
was also studied. A constant rate of twist is considered, where $\theta=0^{\circ}$ at root and $\theta=15.026^{\circ}$ at free end (tip). The frequencies of free vibrations of blade are calculated and compared to the reference values obtained from a 600-element mesh model based on classical FEM (Table 5.13). Based on a six-element DFE analysis, and evaluating the first 5 natural frequencies, an excellent average error $<0.1 \%$ was obtained.

Table 5.13: The triply coupled bending-bending-torsion natural frequencies of the twisted rotating blade, $\Omega=360(\mathrm{rpm})$

\begin{tabular}{cccc}
\hline $\begin{array}{c}\text { Natural Frequency } \\
(\mathrm{rad} / \mathrm{s})\end{array}$ & $\begin{array}{c}\text { FEM } \\
n=600\end{array}$ & $\begin{array}{c}\text { DFE } \\
n=6\end{array}$ & $\begin{array}{c}\text { Error of DFE } \\
(\%)\end{array}$ \\
\hline$\omega_{1}$ & 47.56 & 47.51 & 0.10 \\
$\omega_{2}$ & 71.67 & 71.64 & 0.03 \\
$\omega_{3}$ & 132.60 & 132.26 & 0.26 \\
$\omega_{4}$ & 172.25 & 172.25 & 0.00 \\
$\omega_{5}$ & 268.72 & 268.90 & 0.07 \\
\hline
\end{tabular}

Finally, in order to validate the DFE method for blades and beams of more complex geometries, the blade as discussed in the previous example was re-investigated when the uniform geometry and pre-twisted geometry were modified to model a nonuniform pre-twisted beam. The cross-sectional properties vary along the blade length when the blade is divided into five uniform segments with equal lengths. The flexural and torsional rigidities, $E I_{\eta}, E I_{\xi}$ and $G J$, and also inertia parameters, $\boldsymbol{m}, \boldsymbol{m .} \boldsymbol{k}_{\boldsymbol{m} \boldsymbol{I}}{ }^{2}$ and $\boldsymbol{m} \cdot \boldsymbol{k}_{\boldsymbol{m}}{ }^{2}$, are decreased by $5 \%$ in each segment as shown in Figure 5.16. " $c$ " is the element geometric coefficient multiplied by the reference rigidities and inertia parameters (at root). 


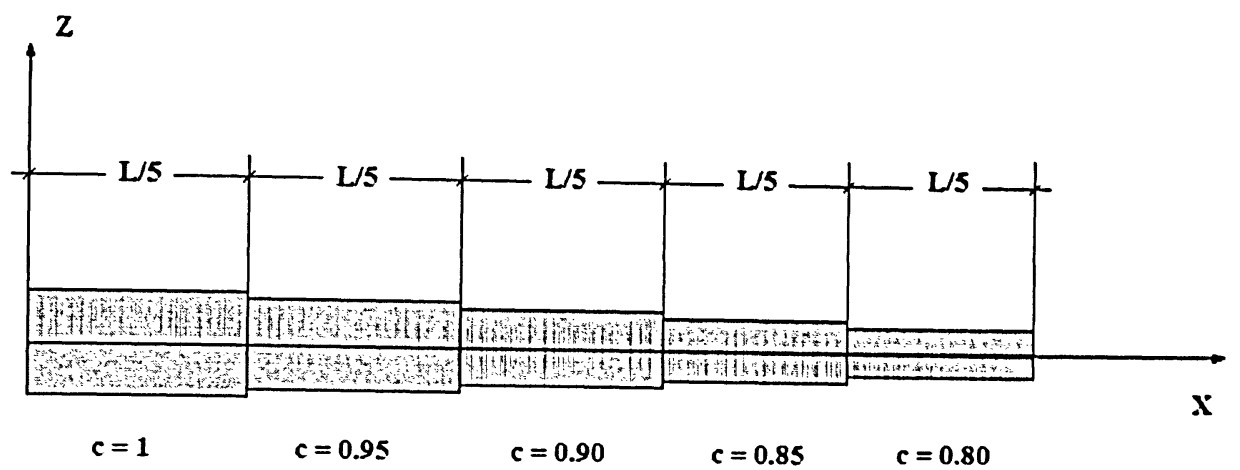

Figure 5.16: The model for piecewise uniform beam.

The results for the natural frequencies of the beam (Figure 5.16) are calculated and shown in Table $5.13(\Omega=360(\mathrm{rpm}))$.

Table 5.14: The triply coupled bending-bending-torsion natural frequencies of the piecewise uniform twisted rotating blade, $\Omega=360(\mathrm{rpm})$

\begin{tabular}{cccccc}
\hline $\begin{array}{c}\text { Frequency } \\
(\mathrm{rad} / \mathrm{s})\end{array}$ & $\begin{array}{c}\text { Reference } \\
\text { FEM }, n=800\end{array}$ & $\begin{array}{c}\text { DFE } \\
n=10\end{array}$ & $\begin{array}{c}\text { Error of DFE } \\
(\%)\end{array}$ & $\begin{array}{c}\text { FEM } \\
n=200\end{array}$ & $\begin{array}{c}\text { Error of FEM } \\
(\%)\end{array}$ \\
\hline$\omega_{1}$ & 48.54 & 48.51 & 0.06 & 48.63 & 0.20 \\
$\omega_{2}$ & 79.49 & 79.46 & 0.03 & 79.56 & 0.09 \\
$\omega_{3}$ & 132.46 & 132.36 & 0.08 & 132.81 & 0.26 \\
$\omega_{4}$ & 181.00 & 181.00 & 0.00 & 181.00 & 0.00 \\
$\omega_{5}$ & 272.59 & 272.52 & 0.03 & 272.97 & 0.14 \\
\hline
\end{tabular}

As it can be seen (Table 5.14) the DFE convergence to the reference values, obtained from an 800-elements FEM model, with much higher rates compared to FEM. As it can be observed (Table 5.14), based on a ten-element DFE model, one can confidently evaluate the first five natural frequencies of the given beam configuration with an average error of les than $0.04 \%$. 
All examples of this chapter along with numerical results of Chapter 4 demonstrate the precision and the practical applicability of the finite element method (FEM) as a strong tool in investigating the dynamic behaviour of non-rotating and rotating coupled beams. Also a comparison between FEM and DFE reveals that the DFE method, along with Wittrick-Williams root counting approach as a robust and accurate solution tool, provides higher convergence rates and lower computation time and cost, especially when multiple and/or higher frequencies are sought. 


\section{CHAPTER 6: CONCLUSION}

\subsection{Concluding Remarks}

For many engineering problems, dealing with flexible structures, the vibration analysis of the system is a vital part of design. Neglecting the dynamic behaviour of the structure could cause catastrophic results and failure in a system. Finding the first few natural frequencies and mode shapes of a flexible structure helps the designer to have better understanding about dynamic behaviour of the structure and to be able to predict the response of the system to excitation such as periodically variable forces or movements.

This research work focused on the free vibration analysis of non-rotating and rotating beams. There are many flexible structures in aerospace engineering which can be modeled by a beam or a combination of beams, such as aircraft wings, propeller blades, satellite antenna and solar panels, compressor, turbine and helicopter blades, etc. All these structure have one character in common: due to the special geometry or material of the structure, they undergo coupled displacements governing coupled differential equations of motion. The analytical solutions for uncoupled equations governing bending and torsion displacements can be found in many vibrations text books, while for the case of coupled vibrations, the analytical solution is not always available.

In vibration analysis of rotating beams, the most complete models have three coupled differential equations for in-plane bending, out-of-plane bending and torsional displacements and an uncoupled equation for longitudinal displacements. The equation governing the beam's axial displacements is uncoupled and can be solved analytically using various methods [24]. For triply coupled beam equations, there have been different numerical approaches proposed by many researchers attempting to find the best solution for the free vibrations response of the system $[2,4,5,6,16]$. Is this research the FEM method, a powerful and accurate method, was first used to study the coupled beam vibrations. The Galerkin weak (integral) formulation, which also satisfies the principle of virtual work, was employed to transform the coupled differential equations of motion into 
algebraic equations and an eigenvalue problem, where the eigenvalues are the natural frequencies and the eigenvectors represent the mode shapes of the system.

Besides developing a conventional FEM method, this research focused specifically on the development of new Dynamic Finite Element (DFE) for bendingbending coupled beams and triply coupled rotating beams, used as a model representing rotary blade dynamics.

As explained in Chapter 5, after discretizing the beam into elements and applying a certain number of extra integration by parts, the ODE's governing the uncoupled beam vibrations appear. Vanishing of these terms leads to the dynamic basis functions of approximation space. A closer look at these terms shows that by application of integration by parts, some integral terms transform to boundary terms, and since the integration in DFE is done numerically, this change can decrease the computation time effectively.

Because of the nonlinear trigonometric nature of the dynamic interpolation functions in the DFE formulation, the root counting procedure presented by Wittrick and Williams $[14,15,26]$ were exploited in order to evaluate the natural frequencies. The approach provides a powerful solution tool for periodic systems with repeated substructures and repeated frequencies [15], in particular. Using thee Wittrick-Williams method, one can calculate directly the $\boldsymbol{i}^{\text {th }}$ frequency $\omega_{i}$ without calculating the frequencies lying below $\omega_{i}$, whereas in the FEM method, the well-known numerical methods frequently used in frequency calculations do not have this feature, even though one could also adapt the W-W algorithm to the standard FEM analysis [35].

The DFE and FEM were then used to investigate the behaviour of coupled vibrations of non-rotating and rotating beam configurations. The examples investigated in this thesis exhibit different aspects of beam vibrations. First, the vibrations of uncoupled beams were studied, where the results showed that solving two or three uncoupled equations together with one nodal displacement vector results in the same results as analytical solutions.

In addition, the effect of an axial load on coupled bending-bending and bendingtorsion beams was studied. As shown in Chapters 4 and 5, the axial load can affect the geometric stiffness of the beam and change the natural frequencies and modes, where a 
tensile force increases the so-called geometric stiffness of the beam and consequently increases the natural frequencies. A compressive axial force, on the other hand, decreases the natural frequencies by softening the beam. Also, when the rotating speed is increased, the centripetal acceleration makes the cord-wise natural frequencies to grow slower than those of beam flap-wise vibration.

The DFE method developed in this research was also advantageously used to determine the natural frequencies and modes of triply coupled beam vibrations. In many engineering design problems, at the preliminary design stage, the designer needs to get a general idea about the dynamic behaviour of the system even before a detailed and rigorous design process or FEM analysis starts. In such cases, having a reliable and accurate simulation tool like DFE helps the designer to better understand the dynamics of a system. Besides, applying the first changes in a trial step is easier and takes much less time with this numerical tool.

\subsection{Comparison Between DFE and FEM}

The DFE, as a finite element approach, follows the same methodology of the conventional FEM, but with some conceptual differences. In the DFE formulation, the Dynamic (frequency dependent) Trigonometric Shape Functions (DTSF's) are exploited to approximate the displacements, while the FEM uses the static (polynomial) shape functions. These polynomial shape functions, cubic for flexural vibrations and linear for torsional vibration, are the solution of static deformations of the beam produced by external forces and moments. The dynamic basis functions used in the DFE formulation are the solutions to the uncoupled ODE's governing the free vibrations of the beam and they are altered such that they reduce to static shape functions when $\omega \rightarrow 0$.

The dynamic character of shape functions in the DFE method causes a higher convergence rate, since the nature of approximation functions are closer than Hermite shape functions to the real solutions. For uncoupled vibrations, the DTSF's are the exact solutions and one can get an infinite number of natural frequencies by a one-element DFE model. 
There is another important difference between two methods: classic FEM leads to a linear eigenproblem with constant mass and stiffness matrices, whereas the DFE formulation results in a nonlinear eigenproblem due to the frequency dependent stiffness matrix which represents both inertia and stiffness of the system.

In general, for a numerical approach some features are more important than others such as reliability, accuracy (or rate of convergence) and computation time. The FEM based formulations have already been proven as reliable and robust methods, and are widely used in engineering and research areas. The reliability of DFE for single axial or bending vibrations and coupled bending-torsion vibrations has also been approved for beams with geometric and/or material coupling [21-25]. The reproduced results for illustrative examples exhibiting different coupled behaviour reconfirmed the excellent reliability of DFE. Besides, the DFE formulation was also extended to the bendingbending and triply coupled beam vibrations and several illustrative examples were presented. Results showed that, as expected, the DFE provides reliable solution for triply coupled vibrations of a beam, and degenerating the dual coupling from triple coupling problems does not affect the robustness of the method.

The accuracy of both DFE and FEM methods is excellent and, as stated in Chapters 4 and 5 , in order to obtain more accurate higher frequencies, the number of elements must be increased. Comparing the convergence results for two methods reveals that for the first and second frequencies, both methods result in the same accuracy, but if higher frequencies are sought, the DFE convergence rates are higher than FEM. The difference between the FEM and DFE methods is more significant when refined DFE (RDFE) is used to model rotating beams or beams with variable cross sections.

The computation time for both methods depends on the number of elements in the model, and since the frequency calculation in the DFE relies on the bisection method, for the same number of elements the computation time for DFE is higher than FEM. A comparison between a combination of accuracy and computation time shows that DFE provides faster solutions. For example, in the case of the coupled bending-bendingtorsion rotating beam (Table 5.11), the average error for the first five natural frequencies obtained from a 7-element DFE model (comparing to a 700-element FEM model) is 0.02 
percent. The DFE computation time, in this case, is less than two minutes whereas the FEM model takes more than two hours to provide the same accuracy.

As a result, despite the differences between the DFE and FEM formulations, both methods can be successfully exploited to carry out the vibration analysis of rotating and non-rotating beams.

\subsection{Future Work}

The research work presented here was focused on the free vibration analysis of homogeneous undamped beams. Euler-Bernoulli bending and St. Venant torsion beam theories were considered in the model. This model neglects the shear deformation and rotary inertia as well as warping and thermal effects. More accurate models consider one, some or all the parameters neglected here; hence, future work can concentrate on increasing the accuracy of the model by including the effects of shear deformation and rotary inertia for triply coupled beams. Also, the warping and thermal effects can be taken into account where needed. To try a realistic model, structural damping also must be taken into account, since all materials incorporate a certain degree of damping. 


\section{Appendix A}

\section{ANALYTICAL SOLUTION FOR BEAMS UNCOUPLED VIBRAITONS}

\section{A.1. Pure Bending Vibrations of a Clamped-Free Beam}

The differential equation of motion for vibrations of the Euler-Bernoulli beam, in which the shear deformation and rotary inertia effects are neglected, can be written as:

$$
\frac{\partial^{2}}{\partial x^{2}}\left(E I(x) \cdot \frac{\partial^{2} W(x, t)}{\partial x^{2}}\right)-\frac{\partial}{\partial x}\left(T(x) \cdot \frac{\partial W(x, t)}{\partial x}\right)+m(x) \cdot \frac{\partial^{2} W(x, t)}{\partial t^{2}}=0
$$

For a clamped-free beam the boundary conditions are:

$$
\begin{gathered}
W(0, t)=0 \\
\frac{\partial W(0, t)}{\partial x}=0 \\
E I \frac{\partial^{2} W(L, t)}{\partial x^{2}}=0 \\
\frac{\partial}{\partial x}\left(E I \frac{\partial^{2} W(L, t)}{\partial x^{2}}\right)=0
\end{gathered}
$$

Using the separation of variables technique, the solution of differential equation is assumed as product of a spatial function $w(x)$ and a harmonic function $\sin \omega t$ :

$$
W(x, t)=w(x) \cdot \sin \omega t
$$


Substituting (A.3) into (A.1):

$$
\left(E I w^{\prime \prime}\right)^{n}-\left(T w^{\prime}\right)^{\prime}-m \omega^{2} w=0
$$

and for a uniform beam:

$$
E I w^{n n}-T w^{n}-m \omega^{2} w=0
$$

Assume:

$$
w(x)=\mathbf{A} e^{\lambda x}
$$

Equation (A.5) becomes:

$$
\begin{gathered}
A \lambda^{4}+B \lambda^{2}+C=0 \\
A=E I ; B=-T ; C=-m \omega^{2}
\end{gathered}
$$

Solving (A.7) for $\lambda$ results in:

$$
\begin{gathered}
\lambda^{2}=\frac{-B \pm \sqrt{B^{2}-4 A C}}{2 A} \\
\lambda_{1,2}= \pm i \sqrt{\frac{\mid-B-\sqrt{B^{2}-4 A C}}{2 A}}= \pm i \alpha \\
\lambda_{3,4}= \pm \sqrt{\frac{-B+\sqrt{B^{2}-4 A C}}{2 A}}= \pm \beta
\end{gathered}
$$

By evaluating the $\lambda$, Equation (A.6) can be written as:

$$
w(x)=\mathbf{A} \sin \alpha x+\mathbf{B} \cos \alpha x+\mathbf{C} \sinh \beta x+\mathbf{D} \cosh \beta x
$$

Applying the boundary conditions (A.2) to find the constants $A, B, C$ and $D$ results in an eigenvalue problem: 


$$
\left[\begin{array}{cccc}
0 & -1 & 0 & 1 \\
-1 & 0 & 1 & 0 \\
-\sin \alpha L & -\cos \alpha L & \sinh \beta L & \cosh \beta L \\
-\cos \alpha L & \sinh \alpha L & \cosh \alpha L & \sinh \beta L
\end{array}\right]\left\{\begin{array}{l}
\mathbf{A} \\
\mathbf{B} \\
\mathbf{C} \\
\mathbf{D}
\end{array}\right\}=\left\{\begin{array}{l}
0 \\
0 \\
0 \\
0
\end{array}\right\}
$$

The characteristic equation of eigenproblem (A.13) can then be written as:

$$
\cos \alpha L \cdot \cosh \beta L=1
$$

For free vibrations of a uniform beam without axial force, $\alpha=\beta$ and:

$$
\omega=\alpha^{2} L^{2} \sqrt{\frac{E I}{m L^{4}}}
$$

The solution of for $\alpha L$, weighted natural frequency, can be found in vibrations text books [34]. The results have been shown in Table A.1:

Table A.1: The weighted and normalized natural frequencies, $\alpha_{n} L$ and $\omega_{n}$, for a cantilever beam obtained by analytical solution

\begin{tabular}{cccc}
\hline \multicolumn{2}{l}{ Weighted Natural Frequency } & \multicolumn{2}{c}{ Normalized natural frequency } \\
\hline$\alpha_{1} L$ & 1.87510407 & $\omega_{1}$ & 3.51601527 \\
$\alpha_{2} L$ & 4.69409113 & $\omega_{2}$ & 22.03449154 \\
$\alpha_{3} L$ & 7.85475744 & $\omega_{3}$ & 61.69721922 \\
$\alpha_{4} L$ & 10.99554073 & $\omega_{4}$ & 120.9019159 \\
$\alpha_{5} L$ & 14.1376839 & $\omega_{5}$ & 199.8741061 \\
$\alpha_{n} L, n>5$ & $(2 n-1) \pi / 2$ & $\omega_{n}$ & {$[(2 n-1) \pi / 2]^{2}$} \\
\hline
\end{tabular}

The natural frequencies have been obtained from equation (A.15). For $E I_{y}=1, m=1$ and $L=1$, the natural frequencies have already been shown in Chapter 4 and 5 . Mode shapes of free vibrations of the clamp-free uniform beam without an axial force can then be calculated by substituting coefficients $A, B, C$ and $D$ into the equation A.12 [34]: 


$$
w_{n}(x)=\cos \alpha_{n} x-\cosh \alpha_{n} x-\frac{\sinh \alpha_{n} L-\sin \alpha_{n} L}{\cos \alpha_{n} L+\cosh \alpha_{n} L}\left(\sinh \alpha_{n} x-\sin \alpha_{n} x\right)
$$

\section{A.2. Torsional Vibrations of a Cantilever Beam}

The governing differential equation of free vibration of beam with St. Venant torsion beam theory can be written as:

$$
\frac{\partial}{\partial x}\left(G J \frac{\partial \Phi(x, t)}{\partial x}\right)-\left(m \kappa_{m}{ }^{2}\right) \frac{\partial^{2} \Phi(x, t)}{\partial t^{2}}=0
$$

For a clamped-free beam the boundary conditions are:

$$
\Phi(0, t)=0
$$

$$
\frac{\partial \Phi(L, t)}{\partial x}=0
$$

Using the separation of variables, the solution of differential equation can be written as:

$$
\Phi(x, t)=\varphi(x) \sin \omega t
$$

By substituting (A.19) into (A.17), one can get:

$$
\left(G J \varphi^{\prime}\right)^{\prime}+\left(m \omega^{2} \kappa_{m}^{2}\right) \varphi=0
$$

For a uniform beam in element coordinates: 


$$
G J \varphi^{\prime \prime}+\left(m \kappa_{m}^{2} \omega^{2}\right) \varphi=0
$$

Assuming an exponential function as the solution of (A.21):

$$
\varphi(x)=A e^{\lambda x}
$$

and substituting (A.22) into (A.21):

$$
\begin{gathered}
G J \lambda^{2}+\left(m \kappa_{m}{ }^{2} \omega^{2}\right)=0 \\
\lambda_{1,2}= \pm i \sqrt{\frac{m \kappa_{m}{ }^{2} \omega^{2}}{G J}}= \pm i \tau
\end{gathered}
$$

One can see that the parameter $\tau$ has already introduced in Chapter 5 (equation (5.4)). The term $l_{e}$ appears in expressions for $\alpha, \beta$ and $\tau$ in equations (5.3) and (5.4) but not in (A.10), (A.11) and (A.24), because in the former equations the solutions are evaluated in terms of element coordinate and $\boldsymbol{l}_{\boldsymbol{e}}$ (the Jacobian) is produced after each differentiation. Substituting equation (A.24) into (A.22), one can get the mode shapes of torsion vibrations of the beam:

$$
\varphi(x)=\mathbf{A} \sin 2 x+\mathbf{B} \cos 2 x
$$

For clamped-free boundary conditions (A.18), an eigenproblem of the following form is obtained:

$$
\left[\begin{array}{cc}
0 & 1 \\
\cos \tau L & -\sin \tau L
\end{array}\right]\left\{\begin{array}{l}
\mathbf{A} \\
\mathbf{B}
\end{array}\right\}=\left\{\begin{array}{l}
0 \\
0
\end{array}\right\}
$$

where the characteristic equation can be written as: 
The weighted natural frequencies, $\tau_{n} L$, and natural frequencies can be simply calculated as:

$$
\begin{gathered}
\tau_{n} L=(2 n-1) \frac{\pi}{2} \\
\omega_{n}=(2 n-1) \frac{\pi}{2} \sqrt{\frac{G J}{L^{2} m \kappa_{m}^{2}}}
\end{gathered}
$$

When $G J=1, L=1, m=1$ and $\kappa_{m}=1$, the analytical solution for torsional natural frequencies was compared with numerical values, calculated by FEM and DFE, in Chapter 4 and Chapter 5, respectively. The mode shapes of torsional vibrations of a cantilever beam then can be written as:

$$
\varphi_{n}(x)=\sin \tau_{n} x
$$




\section{Appendix B}

\section{COMPUTER PROGRAM, ALGORITHMS AND FUNCTIONS}

The formulations presented in this thesis were verified by different illustrative examples discussed in Chapters 4 and 5. Because of some conceptual differences between two approaches, the FEM and DFE method, the algorithms of these two methods are different. The FEM approach, as a well-known and reliable method, has less complexity in programming because of polynomial shape functions and constant mass and stiffness matrices. On the other hand, because of frequency dependent approximation functions, the DFE requires developing more functions and a more complex algorithm.

\section{B.1. The FEM Program and Algorithm}

The FEM method has been programmed first by Maple@ to develop the shape functions vectors and their derivatives. The matrices produced by vector products of shape functions vectors and their derivatives are integrated over element lengths to develop the elementary matrices which construct the mass and stiffness matrices (these matrices are the terms introduced in equations (4.35) to (4.39)). The element mass and stiffness matrices are then calculated. An assembly loop produces the total mass and stiffness matrices. After applying the boundary conditions, the standard eigenvalue command is used to calculate the natural frequencies.

After developing the Maple $\odot$ program, a similar code was written in the Matlab $®$ environment to calculate the natural frequencies and mode shapes. The Matlab® program consists of two functions, the main program calculates the total mass and stiffness matrices and calculates the natural frequencies, and the second function calculates and plots the mode shapes. The Figures B.1 and B.2 show the algorithm of FEM program. 


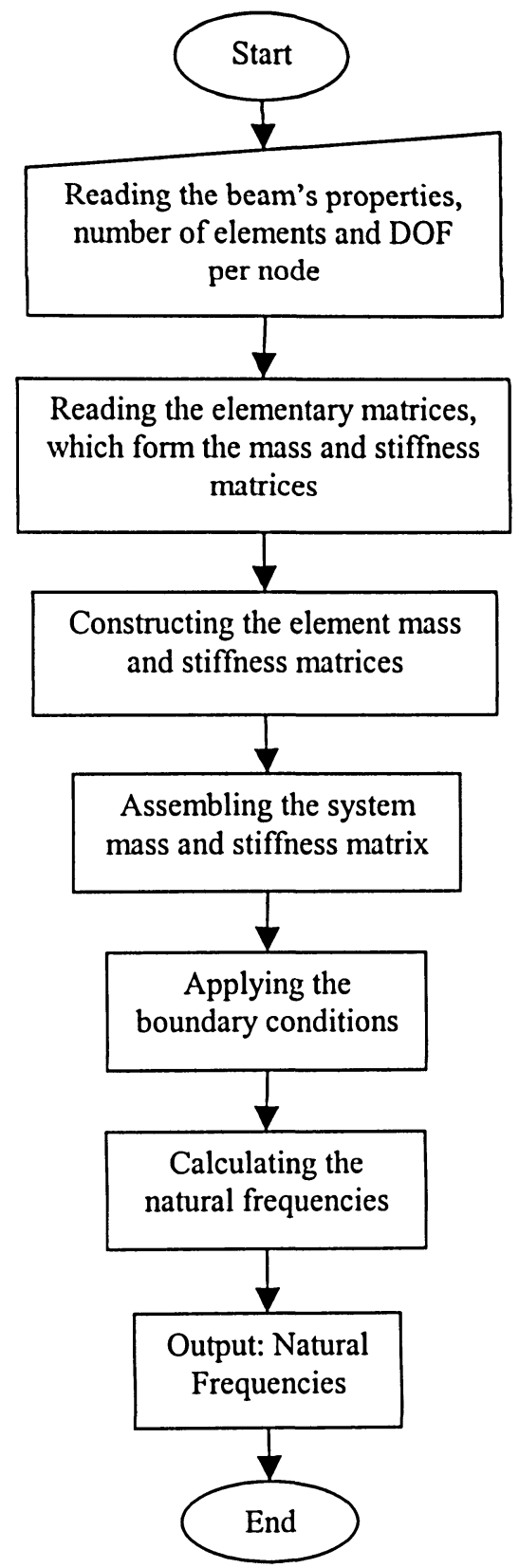

Figure B.1: The Algorithm of main program of FEM method for calculation of natural frequencies. 


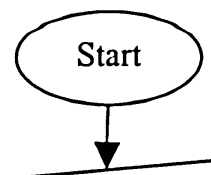

Getting the system mass and stiffness matrices, the number of elements, the number of modes, DOF per node and the natural frequencies

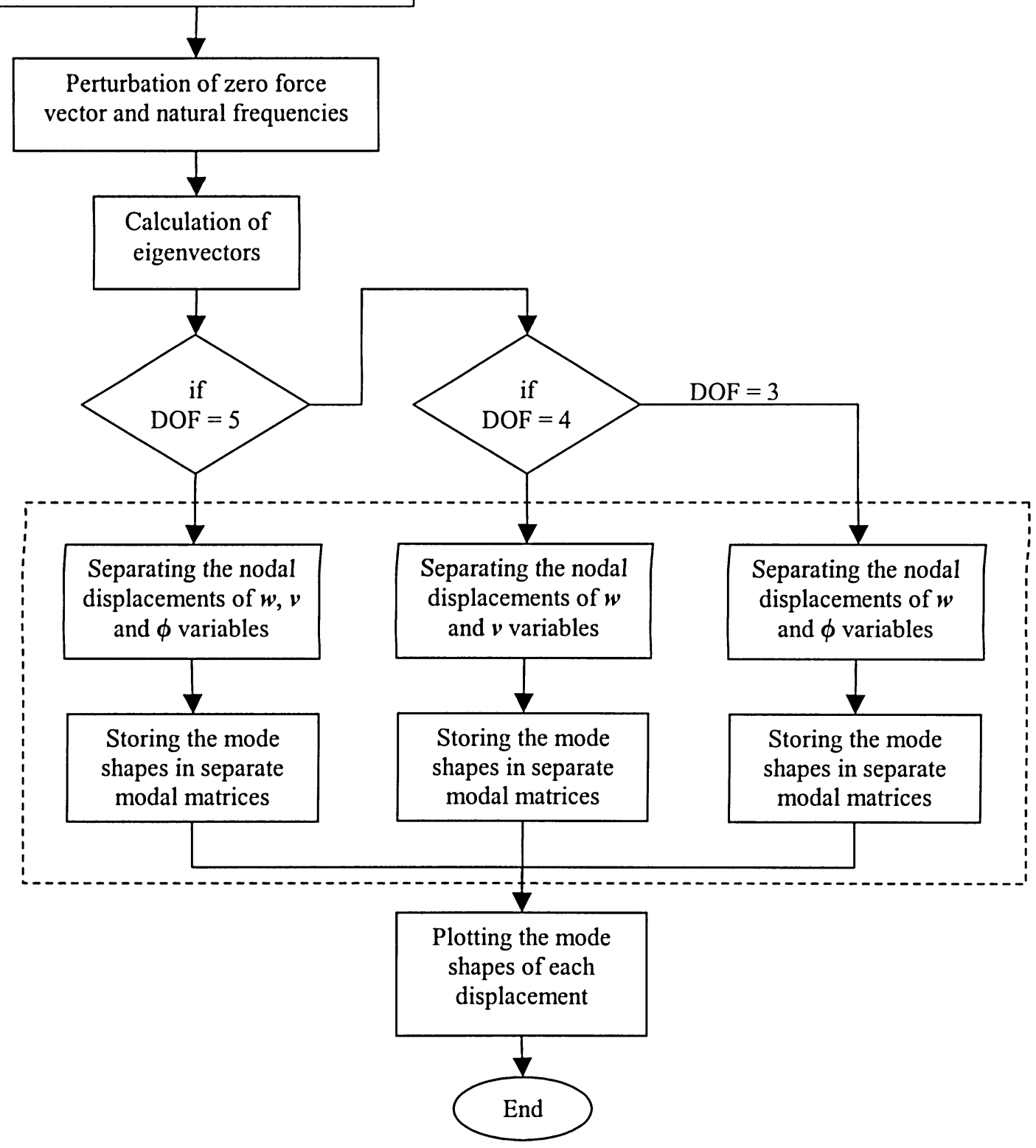

Figure B.2: The Algorithm of the program which calculates the mode shapes, used for the FEM method. 
The DOF in Figure B.2 represents the number of nodal variables, i.e., that means for each bending DOF $=2$ (displacement and slope) and for torsion DOF $=1$. Then DOF $=3$, DOF $=4$ and DOF $=5$ are corresponding to the bending-torsion, bendingbending and bending-bending-torsion vibrations, respectively.

\section{B.2. DFE Program and Algorithm}

As already stated, the mass and stiffness matrices in FEM are constant, but in DFE the elementary matrices and consequently the stiffness matrix are frequency dependent. This causes that the terms in equation (5.35) should be integrated numerically and for each element. On the other hand the nature of bisection method which is an iterative algorithm, require that the calculation of $j$ to be repeated, which causes more computation time. All these reasons result a different algorithm from FEM.

In DFE program, several functions were defined. The main function executes the bisection algorithm to find natural frequencies. In this function another function $J_{0}(\omega)$ is called to calculate $j$ number for each trial frequency.

The main function algorithm has been shown in Figure B.3. 


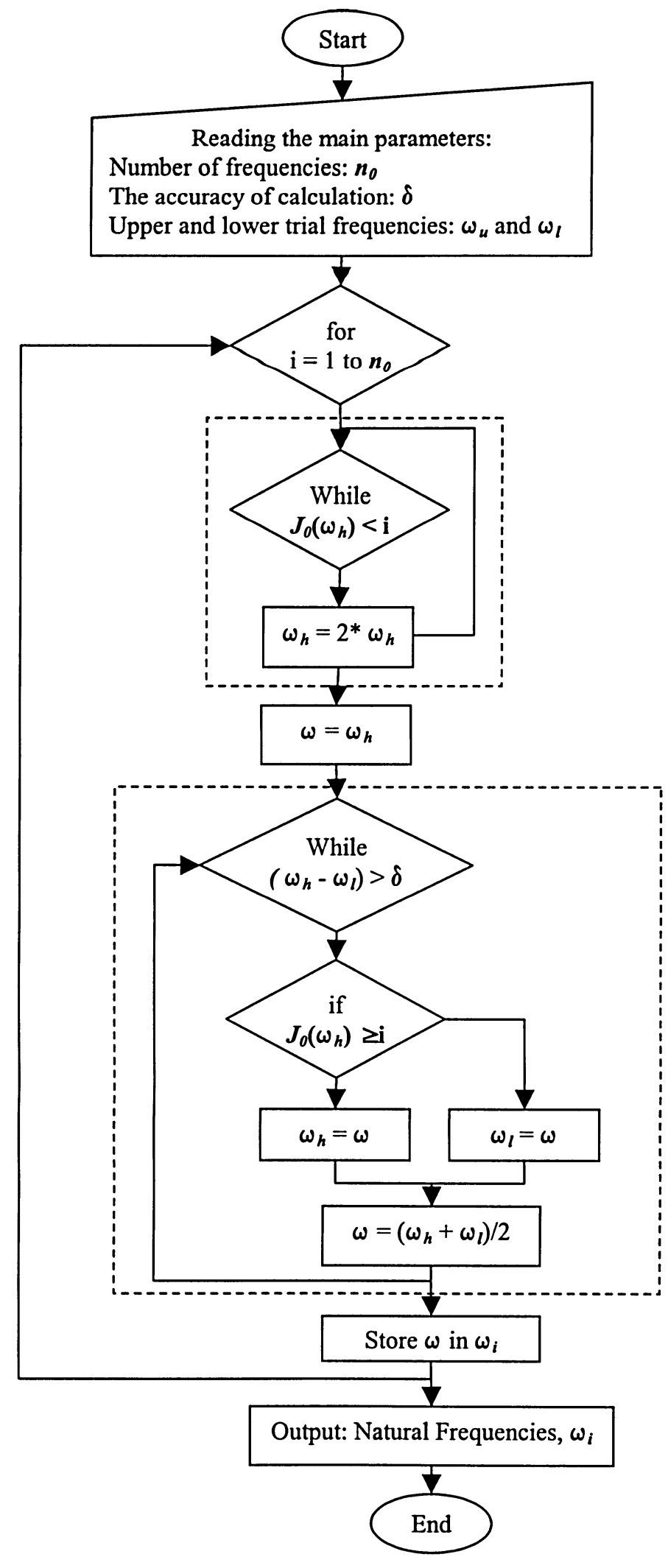

Figure B.3: The main DFE function bisection algorithm. 
Following the Wittrick-Williams method, the function $J_{0}(\omega)$ (used in bisection - algorithm) calculates $\boldsymbol{j}$. All parameters associated with $\boldsymbol{j}$, such as upper triangular stiffness matrix, its sign count and $j_{m}$, are calculated in $J_{0}(\omega)$.

The function gets the beam properties, number of elements, and DOF of the element (as input) and calculates the parameters of equations (5.3) to (5.5) using the trial frequency $\omega^{*}$ ( $\omega^{*}$ is the function's argument which is called from main function). Then the function calculates the uncoupled and coupled element stiffness matrices, equation (5.36) and (5.37). The element stiffness matrix is then calculated along with the $j_{m}$ of the element. After assembling the total stiffness matrix, the geometrical boundary conditions are applied. The result is the matrix, $[K(\omega)]$, which form the nonlinear eigenproblem.

$[K(\omega)]$ is transformed to an upper triangular matrix by a function called UT which gets the matrix and its size as input and returns the upper triangular matrix. Then the sign count of transformed matrix in calculated by another function called SCOUNT. Sign count of total stiffness matrix and $j_{m}$ are added together to calculate $j$ as $J_{0}(\omega)$ 's output.

The algorithm of function $J_{0}(\omega)$ is shown in Figure B.4. There are two functions called in $J_{0}(\omega)$ to calculate the element uncoupled and coupled stiffness matrix. These functions are shown in Figure B.4 as K_COUPLED and K_UNCOUPLED. 


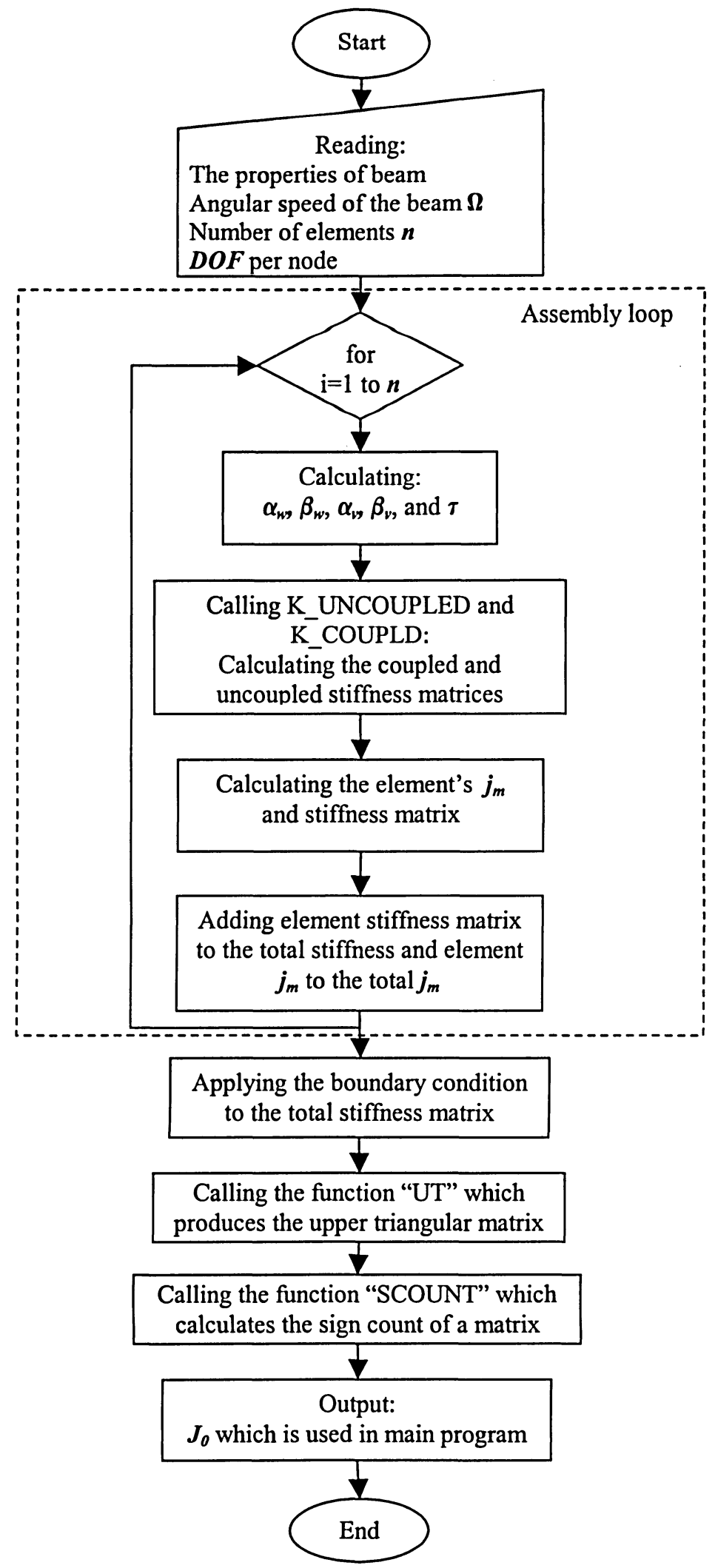

Figure B.4: The algorithm of calculation of $J_{0}(\omega)$. 
The DFE algorithm can be used to evaluate any single $i^{\text {th }}$ natural frequency of beam's free vibrations. Also it is possible to evaluate $n$ natural frequencies, $i$ to $(i+n)$, which is one of the advantages of this method.

When the natural frequencies are evaluated, a function (similar to the one presented in Figure B.2) can be exploited to evaluate the mode shapes corresponding to the natural frequencies.

The algorithms and flow charts, presented in this Appendix for the FEM and DFE methods, can be used for analysis of pure bending, dually or triply coupled vibrations of rotating and non-rotating beams and blades. As already stated, the FEM algorithm is simple and easy to implement, because of polynomial shape functions and constant mass and stiffness matrices. On the other hand, the DFE, because of the iterative nature of Wittrick-Williams and bisection method, is more complicated. This algorithm works with more functions, some of them store the symbolic forms of different terms of stiffness matrix. These symbolic matrices are called in numeric integration loop in K_COUPLED and K_UNCOUPLED functions. 


\section{REFERENCES:}

[1] J.C. Houbolt, and G.W. Brooks, "Differential equations of motion for combined flapwise bending, chordwise bending, and torsion of twisted nonuniform rotor blades." NACA Technical Note 3905, report 1346, 1956.

[2] V.R. Murthy, "Dynamic characteristics of rotor blades." Journal of Sound and Vibration, 49(4) 483-500, 1976.

[3] V.R. Murthy, "Dynamic characteristics of rotor blades: Integrating Matrix Method." American Institute of Aeronautics and Astronautics Journal, 15(4) 595597, 1977.

[4] V.R. Murthy, and A.M. Joshi, "Free Vibration Characteristics of Multiple Load Path Blades by the Transfer Matrix Method." Journal of American Helicopter Society, 31(5), 43-50, 1986.

[5] K.W. Lang, and S. Nemat-Nasser, "An Approach for Estimating Vibration Characteristics of Nonuniform Rotor Blades." AIAA Journal 17, 995-1002, 1979.

[6] P.J. Magari, L.A. Shultz, and V.R. Murthy, "Dynamics of Helicopter rotor blades.” Computers and Structures, 29(5), 763-776, 1988.

[7] J.R. Banerjee, "Coupled bending-torsion dynamic stiffness matrix for beam element.” Int. J. Numer. Meth. Eng. 28, 1283-1298, 1989.

[8] J.R. Banerjee, and F.W. Williams, "Clamped-clamped natural frequencies of a bending-torsion coupled beam." Journal of Sound and Vibrations. 176(3), 301306, 1994.

[9] J.R. Banerjee, and A. J. Sobey, "Energy expressions for rotating tapered Timoshenko beams." Journal of Sound and Vibration, 254(4), 818-822, 2002.

[10] J.R. Banerjee, "Explicit frequency equation and mode shapes of a cantilever beam coupled in bending and torsion" Journal of Sound and Vibration, 224(2), 267-281, 1999.

[11] J.R. Banerjee, "Free vibration of centrifugally stiffened uniform and tapered beams using the dynamic stiffness method" Journal of Sound and Vibration, 233(5), 857-875, 2000.

[12] J.R. Banerjee, "Dynamic stiffness formulation and free vibration analysis of centrifugally stiffened Timoshenko beams" Journal of Sound and Vibration, 247(1), 97-115, 2001. 
[13] S.H.R. Eslimy-Esfahany, and J.R. Banerjee, "Use of generalized mass in the interpretation of dynamic response of bending-torsion coupled beams." Journal of Sound and Vibration, 238(2), 295-308, 2000.

[14] F.W. Williams, and W.H. Wittrick, "An automatic computational procedure for calculating natural frequencies of skeletal structures." International Journal of Mechanical Science, 12, 781-791, 1970.

[15] W.H. Wittrick, and F.W. Williams, "A general algorithm for computing natural frequencies of elastic structures." Quarterly Journal of Mechanics and Applied Mathematics, 24, 263-284, 1970.

[16] G. Surace, V. Anghel, and C. Mares, "Coupled bending-bending-torsion vibration analysis of rotating pre-twisted blades: An integral formulation and numeric examples. Journal of Sound and Vibration, 206(4) 473-486, 1997.

[17] G. Surace, L. Cardascia, and V. Anghel, "A finite element formulation for modal analysis of twisted rotating elastic beams." Meccanica, 32, 377-380, 1997.

[18] A. Arpaci, S.E. Bozdag, and E. Sunbuloglu, "Triply coupled vibrations of thinwalled open cross-section beams including rotary inertia effects." Journal of Sound and Vibration, 260, 889-900, 2003.

[19] K.B. Subrahmanyam, S.V. Kulkarni, and J.S. Rao, "Application of the Reissner method to derive the coupled bending-torsion equations of dynamic motion of rotating pretwisted cantilever blading with allowance for shear deflection, rotary inertia, warping and thermal effects." Journal of Sound and Vibration, 84(2), 223$240,1982$.

[20] L. Jun, S. Rongying, H. Hongxing, and J. Xiangding, "Coupled bending and torsional vibration of axially loaded Bernoulli-Euler beams including warping effects." Applied Acoustics, 65, 153-170, 2004.

[21] S.M. Hashemi, M.J. Richard, and G. Dhatt, "A new dynamic finite element (DFE) formulation for lateral free vibrations of Euler-Bernoulli rotating beams using trigonometric shape functions." Journal of Sound and Vibrations, 220(4), 601-624, 1999.

[22] S.M. Hashemi, and M.J. Richard, "A dynamic Finite Element (DFM) method for free vibrations of bending-torsion coupled beams." Aerosp. Sci. Technol, 4, 41$55,2000$.

[23] S.M. Hashemi, and M.J. Richard, "Free vibrational analysis of axially loaded bending-torsion coupled beams: a dynamic finite element." Computers and Structures, 77, 711-724, 2000. 
[24] S. M. Hashemi, "The Use of Frequency Dependent Trigonometric Shape Functions in Vibration Analysis of Beam Structures-Bridging Gap Between FEM and Exact DSM Formulations", Asian Journal of Civil Engineering, 2 (3\&4)3356, 2002.

[25] S.M. Hashemi, and S. Borneman, "Application of Frequency Dependent Trigonometric Shape Functions in the Vibration Analysis of Laminated Composite Beams." The Fourth Canadian-International Composites Conference (cancom), proceedings. 2003.

[26] W. H. Wittrick, and F. W. Williams, "Exact Buckling and Frequency Calculations Surveyed.” J. Struct. Eng. ASCE, 109, pp. 169-187, 1983.

[27] W. F. Hunter, "Integrating-matrix method for determining the natural vibration characteristics of propeller blades." NASA Technical Note, NASA TN D-6064, 1970.

[28] M. Shavezipur, and S.M. Hashemi, "Application of frequency dependent interpolation functions to the solution of triply coupled differential equations governing flap-lag-torsion beam vibrations." Proceedings of International Conference for Upcoming Engineers (ICUE), Toronto, Canada, 2004.

[29] S.M. Hashemi, and M. Shavezipur, "A Dynamic Finite Element for Free Vibration Analysis of Triply Coupled Blades." $46^{\text {th }}$ AIAA/ASME/ASCE/ AHS/ASC Structure, Structural Dynamics \& Materials Conference, 18-21 April 2005, Austin, Texas, USA (submitted).

[30] M. Tanaka, and A.N. Bercin, "Finite element modeling of the coupled bending and torsional free vibration of uniform beams with an arbitrary cross-section." Applied Math. Modelling, 21, 339-344, 1997.

[31] G. Dhatt, and G. Touzot, "The Finite Element Method Displayed." John Wiley \& Sons, 1984.

[32] D.L. Logan, "A First Course in the Finite Element Method, 3rd edition." Brooks/Cole 2002.

[32] K.J. Bathe, "Finite Element Procedures in Engineering Analysis." Prentice-Hall, Inc., Englewood Cliffs, New Jersey 07632, 1982.

[34] D. J. Inman, "Engineering vibration." Prentice-Hall, Inc., Englewood Cliffs, New Jersey 07632, 1994.

[35] A. Roach, and S. M. Hashemi, "The use of dynamic trigonometric shape functions in coupled torsion-axial vibrations of structural elements- bridging the 
gap between FEM and exact formulation." Proceedings of International Conference for Upcoming Engineers (ICUE), Toronto, Canada, 2003. 